

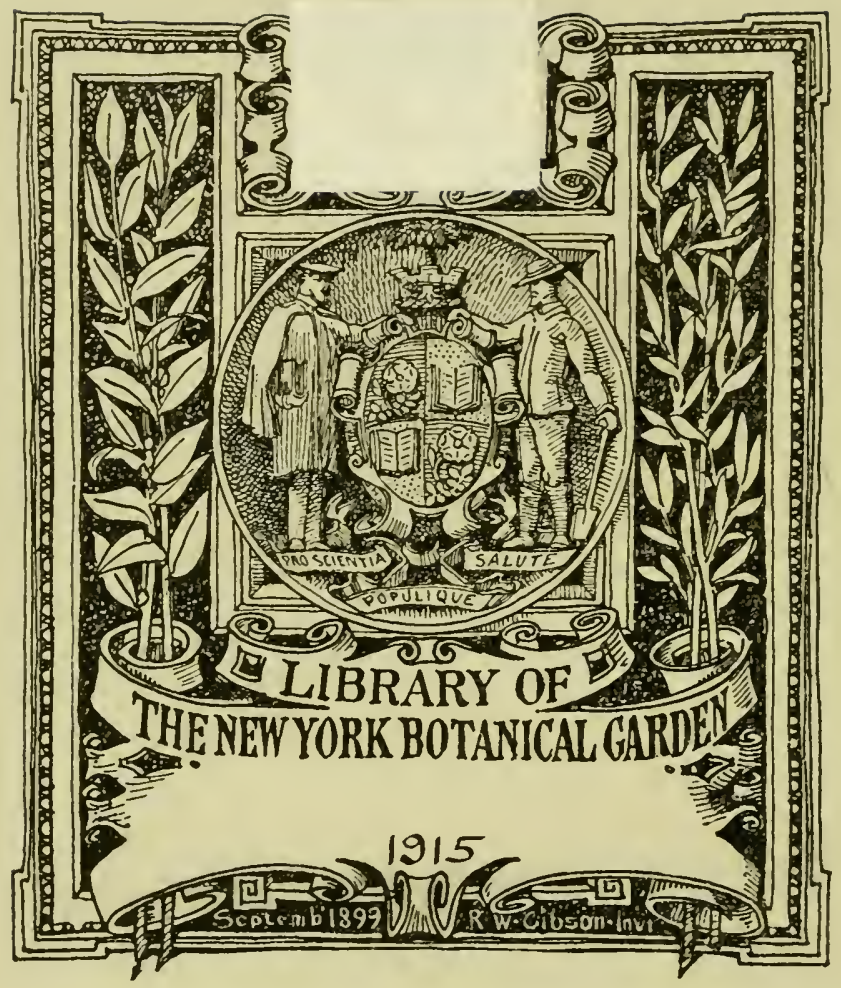







\title{
Untersuchungen über den
}

\section{Phototropismus}

VON

\author{
W. H. ARISZ.
}

Extrait du Recueil des Travaux botaniques Nérlandais. Vol. XII. Livr. 1. 1915.

M. DE WAAL. - 1915. - GRONINGEN. 


\title{
Untersuchungen über den Phototropismus
}

\author{
von \\ W. H. ARISZ. \\ T E I L I. \\ Einseitige Beleuchtungen.
}

\section{ABSCHNITT I.}

Die positive Reaktion.

\section{$\S 1 . \quad$ Einleitung. $\left.{ }^{1}\right)$}

Der Zweck dieser Untersuchungen war die Stimmungserscheinungen $z u$ studieren. Die bekannten Arbeiten Pringsheims hatten es wahrscheinlich gemacht, dasz man hier mit einer jener Eigenschaften zu tun hatte, welche sich nicht zu den Pflanzen beschränken, sondern sich zu allen lebenden Wesen ausdehnen. Nicht nur die Änderung der Reizbarkeit bei niederen und höheren Pflanzen, bei Einzelligen und Keimpflanzen der Phanerogamen, sondern

1) Diese Arbeit ist eine nur wenig geänderte Ulebersetzung der „Onderzoekingen over Fototropie" Proefschrift. Utrecht. 1914.

Vorläufige Mitteilungen sind erschienen in "Proceedings Koninklijke Akademie van Wetenschappen." Amsterdam.

On the connection between stimulus and effect in phototropic curvatures of seedlings of Avena sativa. Meeting of March 25. 1911. Positive and negative phototropic curvatures of seedlings of Avena sativa. Meeting of Sept. 27. 1913.

Adjustment to light in oats. Meeting of Nov. 29. 1913. 
auch jene bei Tieren, wie sie durch die Loebschen Untersuchungen bei Balanuslarven bekannt geworden sind und sogar die Adaptationserscheinungen beim menschlichen Auge würden auf einem selben Prinzip beruhen.

Um diesen Stimmungsprozesz mit Frucht analysieren zu können, zeigte es sich erwünscht über die einfacheren Reizvorgänge näher orientiert zu sein. Die Beobachtungen, welche zu diesem Zweck ausgeführt worden sind beziehen sich alle auf einseitige Reizungen und sind im ersten Teile zusammengebracht worden. Im zweiten Teile ist ein Anfang damit gemacht die Stimmungserscheinungen zu studieren. Die Reihenfolge, worin aber hier die Ergebnisse betrachtet worden sind, ist der bei der Untersuchung befolgten genau entgegengesetzt. Die Resultate, welche bei zweiseitiger Beleuchtung erhalten wurden, haben es möglich gemacht die allseitigen Reizungen näher zu analysieren, und wenn auch jetzt noch nicht alle Data $z u$ einer vollständigen Uebersicht über die Vorgänge bei Kombination verschiedener Reize vorhanden sind, so ist doch der Weg betreten, der dazu bringen musz.

Im dritten theoretischen Teile sind, so weit es bis jetzt möglich ist, die Ergebnisse für die allgemeine Reizphysiologie verwertet worden.

Der gröszte Teil dieser Untersuchungen hat in Utrecht im Institute des Herrn Professor Went stattgefunden. Für seine Anregung und für die Hilfe, womit er mich unterstützt hat und die Liebenswürdigkeit, womit er immer bereit war eine Verbesserung in den Versuchen zu ermöglichen, zolle ich ihm auch hier meinen Dank. Auch Herrn Prof. Moll, der mir das Arbeiten mit dem van Harreveldschen Klinostaten in seinem Laboratorium in Groningen gestattete, bin ich sehr verbunden.

$\S 2$. Allgemeine Methode.

Alle Versuche welche in dieser Untersuchung beschrie- 
ben worden sind, sind mit Keimlingen von Avena sativa verrichtet. Viele reizphysiologische Untersuchungen der letzten Zeit sowohl auf phototropischem wie auf geotropischem Gebiete haben mit diesen Keimpflanzen stattgefunden. Die Folge ist, dasz in diesem Augenblicke Avena eine der best bekannten Pflanzen geworden ist. Die Untersuchungen, welche hier mitgeteilt werden, sind nicht auf andere Pflanzen z. B. Einzellige ausgedehnt worden, da es bevorzugt wurde bei einer Pflanze so tief wie möglich in die verwickelten Reizreaktionen durchzudringen. Mit dem Experimentieren mit den einzelligen Sporangienträger von Phycomyces waren, wie sich bei einer vorläufigen Untersuchung gezeigt hatte, sehr viele Schwierigkeiten verbunden; übrigens hätten diese vielleicht den Vorzug vor den durch ihren vielzelligen Bau so viel komplizierteren Avenakeimlingen verdient. Der Hafer, womit der gröszte Teil dieser Untersuchungen ausgeführt worden ist, rührte von $\mathrm{Sv}$ alöf her. Die verschiedenen untersuchten reinen Linien besaszen keinen ins Auge fallenden Unterschied in Lichtempfindlichkeit. Sie reagierten äuszerst gleichmäszig wahrscheinlich in Folge des gleichmäszigen Wachstums des Materiales.

Die Weise, worauf die Pflanzen gezogen wurden, weicht nicht von der Methode früherer Untersucher ab. Sie ist ausführlich von Rutgers $\left.{ }^{1}\right)$ beschrieben worden ( $\$ 5$ p. 22 und 23).

Es gibt eine Erscheinung, welche jedem der mit Hafer experimentiert hat bekannt ist, die hier besprochen werden musz: das Auswachsen der Hypokotylen. Rutgers suchte die Ursache im Mangel frischer Luft und in der schädlichen Wirkung der Laboratoriumluft. Bla a u w ${ }^{2}$ ) war im Frühjahr

1) Rutgers, A. A. L. The influence of temperature on the geotropic presentation time. Recueil d. Trav. Bot. Néerl. Vol. IX. 1912.

2) Blaauw, A. H. Die Perzeption des Lichtes. Recueil d. Trav. Bot. Néerl. V. 1909. 
dadurch belästigt. In Uebereinstimmung mit Noack ${ }^{1}$ ) kann diese Erscheinung dem Feuchtigkeitszustande zugeschrieben werden. Das starke Auswachsen der Hypokotylen in feuchter Luft weist aber darauf, dasz es nicht wie Noack meint durch Mangel an Feuchtigkeit verursacht wird. Bei sehr groszer Feuchtigkeit des Bodens und in mit Wasserdampf gesättigter Luft wachsen die Hypokotylen sehr stark und vollkommen regelmäszig aus. Sie können unter diesen Umständen etwa 2 cM. lang werden. Bei ungleichmäsziger Feuchtigkeit ist das Auswachsen mehr einseitig. wobei die von dem Korn abgewendete Seite am meisten begünstigt wird.

Das Material für alle Versuche ist im Laboratoriumsgewächshause, das sich im Hortus academicus befindet, gezogen worden. Die Pflanzen haben sich also immer in reiner Luft entwickelt.

Aufstellung. Die Versuche, welche hier mitgeteilt werden, erstrecken sich über mehrere Jahre. In dieser Zeit ist fortdauernd Verbesserung der Aufstellung angestrebt worden. Die Folge davon ist, dasz die Umstände worunter die verschiedenen Versuchsreihen stattgefunden haben nicht vollkommen mit einander zu vergleichen sind. Der gröszte Teil der Versuche ist in Utrecht angestellt. eine Reihe ergänzender Bestimmungen insbesondere um den van Harreveldschen Klinostaten zu benutzen, im botanischen Institute in Groningen. Die Aufstellung in den verschiedenen Räumen weicht hauptsächlich in den Lichtquellen ab. In Groningen wurden elektrische Metallfadenlampen von 500 und $100 \mathrm{H}$. K. benutzt. Da diese auf einer Akkumulatorenbatterie bei konstantem Voltage brannten, gaben sie ein konstantes Licht. In Utrecht ist für die in $\S 3,4$ und 6 mitgeteilten Versuche, welche

1) Noack, K. Die Bedeutung der schiefen Lichtrichtung für die Helioperzeption parallelotroper Organe. Zeitschrift für Bot. J. 6. 1914. 
im Dunkelzimmer des Laboratoriums stattfanden, eine Lampe mit invert brennendem Gasglühlicht benutzt worden. Diese Autstellung ist von $\mathrm{Blaauw^{1 }}$ ) beschrieben worden. Alle späteren Versuche sind aber in einem kleinen Dunkelzimmer im Laboratoriumsgewächshause genommen worden. Als Lichtquelle diente hier stets eine Nernstprojektionslampe mit drei Stiften, welche auszerhalb des Dunkelzimmers aufgestellt war und durch eine Kühleinrichtung davon getrennt war. Wegen der starken Wechslungen der Netzspannung war es auch mit Hilfe eines Widerstandes nicht möglich tagsüber mit vollkommen konstanter Stromstärke zu arbeiten. Darum sind so viel wie möglich alle Wahrnehmungen abends bei konstanter Lichtstärke wiederholt und kontrolliert worden. Einige Versuche mit sehr groszer Lichtstärke konnten nur mit der im Vorlesungszimmer aufgestellten Projektionslampe mit elektrischem Bogenlichte genommen werden. Am Ende der Untersuchungen insbesondere bei den Versuchen mit zweizeitiger Beleuchtung wo zwei vollkommen konstante Lichtquellen unerläszlich waren, sind Metallfadenlampen von 10 Volt benutzt worden, welche auf Akkumulatorenstrom bei konstantem Voltage brennten. Dies erwies sich als die einzige zuverlässige Methode, immer mit derselben konstanten Lichtstärke zu arbeiten.

Die Lichtstärke ist mit einem Photometer nach Weber bestimmt worden, welchen Prof. Snellen, Direktor des "Nederlandsch Gasthuis voor Ooglijders" so liebenswürdig war zur Benutzung zu überlassen. Die Beleuchtungszeiten sind mit einem Chronometer bestimmt worden.

Es sind noch eine Anzahl Apparate benutzt worden, welche bei der Besprechung der Versuche, wofür sie benutzt wurden, beschrieben worden sind.

Temperatur. Im Dunkelzimmer des Laboratoriums ist man gänzlich von der Temperatur der Umgebung abhän-

1) Blaauw, 1. c. $\$ 2$. 
gig. Das Dunkelzimmer im Gewächshause ist für diese Versuche mit einer einfachen Einrichtung um die Temperatur konstant $z u$ halten versehen worden. Ein Ventilator, der sich für ein geöffnetes Fenster befindet, bläst durch ein Röhrensystem einen Strom frische Luft in das Dunkelzimmer. Eine Vierzahl Lampen, die auf verschiedenen Stellen aufgestellt und in blechernen Büchsen lichtdicht abgeschlossen sind, können eine genügende Verwärmung geben. Durch einen Quecksilberthermoregulator wird, wenn die Temperatur eine bestimmte Höhe erreicht, ein elektrischer Strom geschlossen, der mit Hilfe eines Relais eine Umschaltung zu Stande bringt, wodurch die Verwärmungslampen, welche bis zu diesem Augenblicke gebrannt haben, ausgeschaltet werden und zu gleicher Zeit der Ventilator in Wirkung gestellt wird, der einen Strom kalte Luft von auszen ins Dunkelzimmer führt. Durch das abwechselnd Wirken des Ventilators und der Verwärmungslampen wird eine genügend konstante Temperatur und zu gleicher Zeit eine dauernde Erfrischung der Atmosphäre erreicht. Die gröszten Abweichungen der Temperatur betrugen $1^{\circ} \mathrm{C}$.

Bedingung für ein gutes Funktionieren ist, dasz die Temperatur des Dunkelzimmers höher sein musz als jene der kühlenden Auszenluft. Dadurch ist es nicht möglich im Sommer unter $25^{\circ}$ à $26^{\circ}$ Cels. zu arbeiten. Das Dunkelzimmer im Groninger Laboratorium hatte auch eine Einrichtung für konstante Temperatur. Dieses System beruhte auf das Einblasen kalter oder warmer Luft.

Reine Luft. Ebensowenig wie im Dunkelzimmer in Groningen bestand im Gewächshause in Utrecht Gefahr für Verunreinigung der Luft ${ }^{1}$ ). Das kann nicht vom

1) Richter, O. Ueber die Steigerung der heliotropischen Empfindlichkeit von Keimlingen durch Narkotika. Verh. d. Vers. deutsch. Naturf. u. Ärzte. Münster. 1912. Abt. Botanik.

Knight, J. and Crocker Wm. Toxicity of Smoke, The bot. gaz. 55 , 1913. 
Dunkelzimmer im Institute und vom Vorlesungszimmer gesagt werden. Eine Anzahl Versuche, welche im Dunkelzimmer des Institutes ausgeführt worden sind, werden hier nicht mitgeteilt werden, da nicht genügende Sicherheit besteht, dasz diese Resultate quantitativ zuverlässig sind.

Nutationen. Ein wichtiger Faktor bei der Untersuchung tropistischer Krümmungen sind die vielfach auftretenden Nutationen, worunter hier alle nicht erwünschte Krümmungen verstanden werden. Die autonomen Nutationen von Avena sind von Maillefer ${ }^{1}$ ) ausführlich untersucht worden. Es ist $z \mathrm{u}$ bedauern, dasz seine Untersuchung keine Data enthält über die immer zitierte Mitteilung, dasz die autonomen Nutationen in einer bestimmt orientierten Ebene stattfinden.

Rothert ${ }^{2}$ ) teilt mit, dasz bei Avena Oscillationen des Keimlinges vorkommen. Er suchte die Ursache in einem wechselsweise Ueberwiegen der Schwerkraft und des Phototropismus, er änderte aber seine Meinung, als er sah, dasz die Oscillationen auch auf dem Klinostaten vorkommen. Mit groszer Wahrscheinlichkeit kann diese Erscheinung negativem Phototropismus zugeschrieben werden. Die Abbildungen Rotherts ähneln dem was hier später als kombinierte positive und negative phototropische Krümmungen beschrieben werden wird.

Eine ganz andere Erscheinung hat Rutgers ${ }^{3}$ ) beobachtet. Nach einer negativ geotropischen Schwellenkrümmung, fände wieder eine positive und später wieder eine negative statt. Warum Rutgers diese Erscheinung nicht für identisch mit der sogenannten, geotropischen Ueberkrümmung von Sachs gehalten hat ist nicht deutlich. Eben dasz

1) Maillefer, A. Nouvelle étude expérimentale sur le géotropisme, Bull. Soc. Vaud. Vol. XLVIII. 1912.

$\left.{ }^{2}\right)$ Rothert, W. Ueber Heliotropismus. Cohn's Beitr. z. Biol. d. Pfl. Bd. VII. 1896. p. 32.

3) Rutgers l. c. p. 34 e. s. 
alle Individuen eine Spitzenasymmetrie nach derselben Seite zeigen und alle gleichmäszig reagieren, indem die Nutationsperiode, welche Rutgers bestimmt hat (70 Minuten) etwa 2 mal so grosz ist, wie die Reaktionszeit, weist vielmehr darauf, dasz diese Gegenkrümmungen geotropischer Natur sind. Es braucht nicht $z u$ verwundern, dasz schon eine so kleine Aenderung in der Lage der Spitze perzipiert wird, da nach allen Untersuchern gerade die Spitze der meist empfindliche Teil des Keimlinges ist. Rothert's Fig. $60 § 77$ beruht wahrscheinlich auf Autotropismus.

Man sieht also, dasz man sehr vorsichtig sein musz um nicht Krümmungen welche gar nicht autonom sind als solche $\mathrm{zu}$ betrachten. Es ist nicht zweifelhaft dasz eine grosze Anzahl Ursachen zu aitiogenen Nutationen führen kann. Van der $\left(\mathrm{O}^{1} \mathrm{k}^{\mathrm{l}}\right.$ ) hat ins besondere auf die Kontaktkrümmungen gewiesen. Schon sind die Hypokotylkrümmungen betrachtet worden, welche das Koleoptyl aus der vertikalen Lage führen und eine geotropische Induktion verursachen. Wahrscheinlich sind auch der Autotropismus und ein eventueller asymmetrischer Bau des Keimlinges Ursache des Auftretens von Nutationen. Es versteht sich, dasz durch all diese unerwünschte Krümmungen es äuszerst schwierig wird vollkommen zuverlässiges Material zu bekommen. Auch das Experimentieren wird dadurch belästigt, ins besondere, wenn die Temperatur wobei gearbeitet wird ziemlich hoch ist.

\section{§3. Der Verlauf einer positiven Krümmung.}

Wenn man die ersten Stadien einer Krümmung beobachten will, musz man ein Mikroskop benutzen. A priori würde man geneigt sein $z u$ denken, dasz je stärker die

1) v. d. Wolk, P. C. Investigation on the transmission of light stimuli in the seedlings of Avena. Publications sur la Physiologie Végétale. 1912. 
Vergröszerung sei, je mehr auch die Beobachtungen an Genauigkeit zunehmen müszten. Es hat sich aber gezeigt, dasz dies nicht der Fall ist. Bei starker Vergröszerung ist das Gesichtsfeld relativ klein und das Wachstum macht ein dauerndes Nachstellen unerläszlich; auszerdem sind Koleoptylen, welche gerade wachsen sehr selten. Die gröszte Schwierigkeit entsteht aber dadurch, dasz es bei stärkerer Vergröszerung unmöglich ist festzustellen ob das erste Auftreten einer Abweichung der Spitze wirklich der Anfang einer phototropischen Krümmung ist. Beobachtet man ein Koleoptyl in den ersten Krümmungsstadien mit schwacher Vergröszerung, dann zeigt sich, dasz der Anfang der phototropischen Krümmung nicht so sehr eine $\mathrm{Ab}$ weichung der ganzen Spitze aus ihrer ursprünglichen Lage als eine kleine Aenderung ihrer äuszeren Form ist. Die Spitze hat bei allen Koleoptylen genau dieselbe Gestalt, mehr oder weniger konisch zulaufend; bei einem unbeleuchteten Keimlinge ist sie in ihrer Medianebene immer symmetrisch. Der Anfang der Krümmung musz sich also in einer Asymmetrie der Spitze äuszern. Da das Koleoptyl nicht in eine feine Spitze ausläuft. welche als Index dienen könnte, sondern oben mehr abgerundet ist, kann die Asymmetrie am leichtesten beobachtet werden bei einer Vergröszerung, welche eine Uebersicht über die beiden asymmetrisch werdenden Seiten zuläszt. Bringt man ins Okular des Horizontalmikroskopes ein Netzmikrometer. dann kann das Bild der Spitze sehr genau auf Millimeterpapier nachgezeichnet werden.

Durch Vergleichung auf diese Weise verfertigter Zeichnungen ist es möglich trotz des dauernden Wachsens die Krümmung schon in den Anfangsstadien zu beobachten. Diese Methode hat noch einen Vorteil. Die Asymmetrie der Spitze ist der typische Anfang einer tropistischen Krümmung. Eine geotropische entsteht in derselben Weise. Dadurch ist es möglich sie von anderen Krümmungen, 
meistens Nutationen genannt, zu unterscheiden, welche eine seitliche Abweichung der ganzen Spitze verursachen. Der Anfang der tropistischen Lichtkrümmung ist also auf diese Weise mit ziemlich groszer Sicherheit zu erkennen.

Um einen Maszstab für die Grösze der Krümmung zu haben ist die horizontale Abweichung von der Mitte der abgelenkten Spitze bis zu ihrer ursprünglichen Stelle gemessen worden. Es ist unwahrscheinlich, dasz dieser Maszstab ein genaues Bild aller Einzelheiten der Krümmung gibt. Doch haben auch andere Untersucher ihn benutzt. (Maillefer $\left.{ }^{1}\right)$, Polowzow $\left.{ }^{2}\right)$.) Eine prinzipiell bessere Methode hat Tröndle ${ }^{3}$ ) angegeben. Er teilt die ganze Pflanze in Zonen und miszt die Krümmung jeder Zone einzeln. Abgesehen davon, dasz die Versuche, die hier mitgeteilt werden, schon längere Zeit vor der Erscheinung der Tröndleschen Arbeit beendet waren, würden sehr grosze Schwierigkeiten mit der Anwendung seiner Methode verbunden gewesen sein. Es ist sicher ein Vorteil die Krümmungsstärke, der verschiedenen Zonen jede für sich zu bestimmen. Es wird aber schwer sein die Marken so anzubringen, dasz durch die Berührung keine Kontaktkrümmungen ausgelöst werden und diese Marken bei schachem rotem Lichte $z u$ beobachten. Auch kann diese Methode nur bei starken Krümmungen angewendet werden. Darin stimmt sie mit einer anderen überein, welche früher viel benutzt wurde, wobei die Krümmungsstärke in Graden ausgedrückt wurde. Eine Asymmetrie zu messen ist sie aber unzureichend. Die einfachste Weise, worauf der Krümmungsprozesz untersucht werden kann, ist die

1) Maillefer, A. Nouvelle étude expérimentale sur le géotropisme. Bull. Soc. Vaud. Vol. XLVIII. 1912.

") Polowzow, W. Untersuchungen über Reizerscheinungen 1909.

$\left.{ }^{3}\right)$ Tröndle, A Der zeitliche Verlauf der geotropischen Reaktion und die Verteilung der geotropischen Sensibilität in der Koleoptile. lahrb. Wiss. Bot. Bd. 52. 1913. 


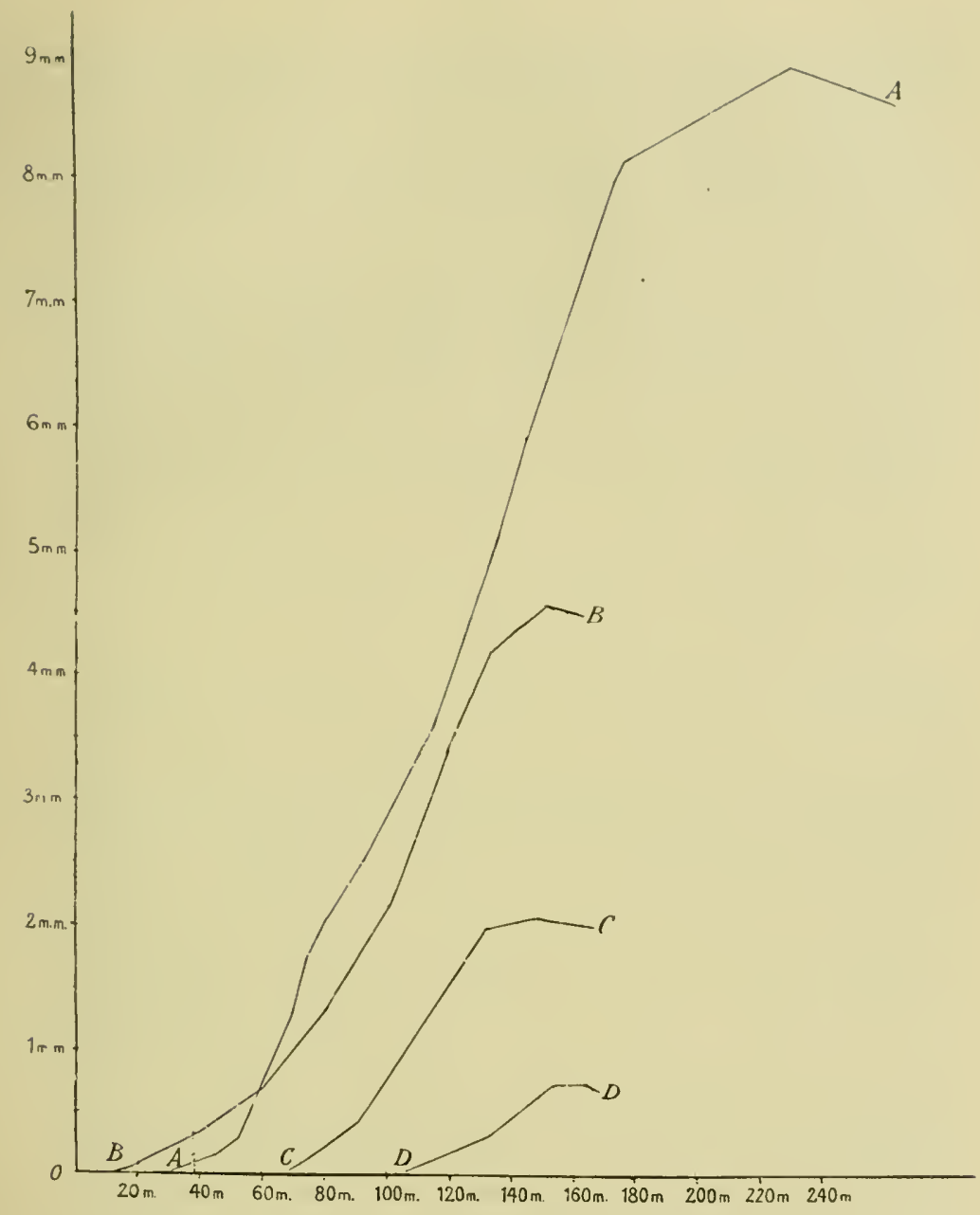

Fig. 1.

Verlauf einer positiven phototropischen Krümmung. Temp. $17,5^{\circ} \mathrm{C}$. A gereizt mit 800 M.K.S. B mit 112 M.K.S. C mit 20 M.K.S. D mit 5 M.K.S.

Auf die Abszisse ist die Anzahl Minuten nach dem Anfange der Reizung auf den Ordinat die horizontale Abweichung in $\mathrm{mM}$. aufgetragen worden

Der Unterschied zwischen A und B wird hauptsächlich durch die verschiedene Länge der Planzen verursacht. 
in oben genannter Weise ausgeführte Bestimmung der horizontalen Spitzenabweichung. Es ist aber selbstverständlich unerläszlich grosze Vorsicht zu gebrauchen, wenn man aus Angaben, welche man auf diese Weise bekommt, Schluszfolgerungen ziehen will.

Fig. 1. gibt die Ergebnisse einiger in oben beschriebener Weise verrichteten Wahrnehmungen. Durch Vergleichung der nach bestimmten Zeiten angefertigten Zeichnungen sind die an diesen Momenten bestehenden horizontalen Abweichungen gemessen worden. Die Kurven zeigen, wie die Stärke der horizontalen Abweichung wächst bei einer Pflanze, welche sich nach einer Reizung der angegebenen Stärke krümmt. Die Beleuchtung der ganzen Pflanze fand immer innerhalb einer Minute statt.

Aus dieser Figur geht hervor, dasz es durch die stärkere Vergröszerung möglich wird den Krümmungsprozesz schon eher zu verfolgen als bei makroskopischer Wahrnehmung. Der Uebergang vom nur mikroskopisch sichtbaren Teil zu den späteren Krümmungsstadien ist sehr allmählich. Nur bei starken Krümmungen gibt es einen mehr oder weniger deutlichen Knick. Nach Blaauw ${ }^{1}$ ) träte hier im Krümmungsprozesse eine neue Phase auf. Die Ursache ist wahrscheinlich, dasz in diesem Augenblicke die sich weiter von der Spitze hefindenden Zonen an der Krümmung teilzunehmen anfangen. Diese Zonen krümmen sich, wahrscheinlich etwas schneller, doch ist dieses nicht sicher begründet, da man bei der Analyse der Kurven von fig. 1 daran zu denken hat, dasz man bei gleicher Differenz in Längewachstum zwischen Vorder- und Hinterseite einer bestimmten Zone eine gröszere Abweichung der Spitze bekommt, wenn diese Zone sich in gröszerer Entfernung von der Spitze befindet. Das wird dadurch verursacht,

1) Blaauw, 1. c. p. 34 . 
dasz derjenige Teil der Pflanze der sich über der sich krümmenden Partie befindet, die Krümmung vergröszert überbringt auf die gemessene horizontale Abweichung.

Die Kurven von Fig. 1 haben alle denselben Verlauf. Nach einer bestimmten Zeit tritt bei allen ein Maximum auf. Um die Bedeutung dieses Maximums zu verstehen musz man bedenken, dasz auch bei kleiner Abweichung der Spitze aus dem Vertikal die Schwerkraft eine Gegenkrümmung induziert. Diese nimmt fortdauernd an Stärke zu sodasz die Geschwindigkeit der phototropischen Krümmung abnehmen musz und eine Maximumkurve entsteht.

§4. Aufhebung dergeotropischen Gegenkrüm mung.

Schon Müller liesz seine Pflanzen um die horizontale Achse eines Klinostaten rotieren um den Einflusz der einseitig arbeitenden Schwerkraft auszuschalten. Unter dem Einflusse Nolls hat man das später als überflüssig betrachtet, weil man sich vorstellte, dasz die Empfindlichkeit für Schwerkraft durch das Licht ausgeschaltet würde. Bei den im Folgenden beschriebenen Versuchen ist nicht ein gewöhnlicher sondern der Fitting-Pfefferschen Klinostat benutzt worden.

Auch wenn man die Pflanzen während gleicher Zeiten in $z w^{-}=1$ Lagen bringt, welche $180^{\circ}$ abweichen, sodas $z$ die Wirkung der Schwerkraft in den auf einander folgenden Lagen sich aufhebt, ist das Zustandekommen einer geotropischen Gegenkrümmung ausgeschlossen. Die Pflanzen wurden gleich nach der Beleuchtung auf den Klinostaten gestellt und mit einem Mikroskope beobachtet, während sie sich in einer der Ruhelagen befanden. Es stellte sich heraus, dasz ein Intermittierungstempo von zwei Minuten, wobei sich die Pflanzen abwechselnd 2 Minuten in den Lagen $+0^{\circ}$ und $-0^{\circ}$ befanden für diese Versuche sehr 
geeignet war. Fig. 2 zeigt in einer Kurve den Verlauf der phototropischen Krümmung bei Ausschaltung der einseitigen Schwerkraftwirkung. So wie bei der Krümmung von Fig. 1 ist auch hier die horizontale Spitzenabweichung gemessen.

Bei diesem Versuche wurde schon 10 Minuten nach

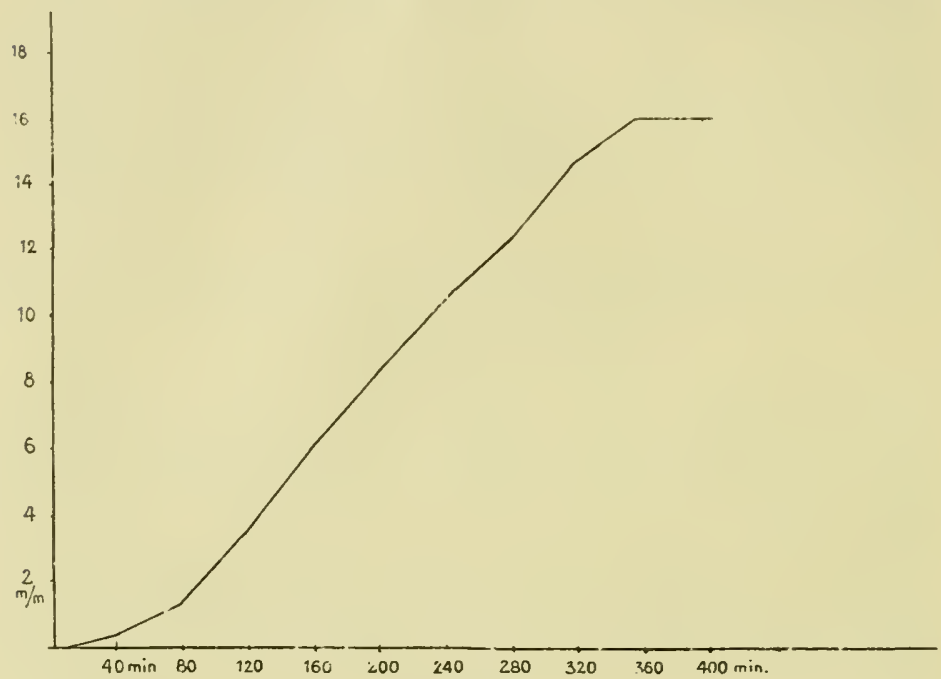

Fig. 2.

Der Verlauf einer positiven phototropischen Krümmung bei einer Pflanze, welche um die horizontale Achse eines Klinostaten rotiert.

Temp. $17,5^{\circ}$ C. Reizstärke 360 M.K.S.

Auf die Abszisse ist die Anzahl Minuten nach dem Anfange der Reizung, auf den Ordinat die horizontale Abweichung der Spitze in mM. aufgetragen worden.

dem Anfange der Reizung eine horizontale Abweichung beobachtet. Auf Grund der Untersuchungen Maillefers ${ }^{1}$ ) über geotropische Krümmungen, wo er gefunden hat, dasz eine Pflanze gleich nach dem Horizontallegen verschiedene Nutationen ausführt, ist es vorsichtiger auf diesen frühen

1) Maillefer, I. c. 
Anfang der Krümmung keinen besonderen Wert zu legen. Aus dieser Kurve geht hervor, dasz während der ersten 6 Stunden die horizontale Abweichung dauernd gröszer wird und nicht wie bei Gegenwirkung der Schwerkraft nur während ungefähr 3 Stunden. Um die Bedeutung dieses Ruhepunktes zu verstehen, der nach ungefähr 6 Stunden auftritt, ist es erwünscht eine Uebersicht über den ganzen Verlauf der Krümmung $z u$ haben. Auch hierfür ist ein intermittierender Klinostat benutzt worden, aber jetzt wurde mittels einer Linse ein Bild der ganzen sich krümmenden Pflanze auf ein durchsichtiges Blatt Papier projiziert. Auch bei schwachem rotem Lichte war es möglich so scharf zu projizieren, dasz das Bild leicht nachzuzeichnen war. Die auf diese Weise bekommene Serie

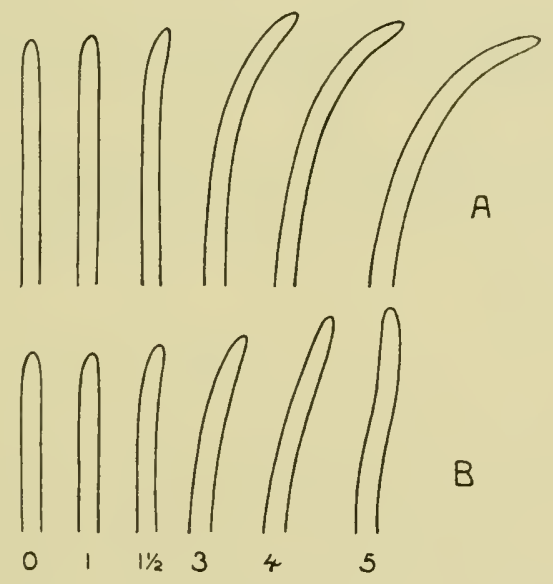

Fig. 3.

Zwei Pflanzen A und B mit einer selben Lichtmenge etwa 25 M.K.S. gereizt bei $17,5^{\circ} \mathrm{C}$.

A. Der Krümmungsverlauf, wenn die einseitige Wirkung der Schwerkraft durch Rotation um die horizontale Achse eines Klinostaten ausgeschaltet worden ist. B. Der Krümmungsverlauf bei Gegenwirkung der Schwerkraft.

Die Zahlen geben die Zeit in Stunden an, welche nach dem Anfange der Beleuchtung verlaufen ist. 
Zeichnungen macht es möglich den Krümmungsverlauf in allen Zonen der Pflanze zu folgen.

In fig. 3 sind zwei Planzen A und B abgebildet welche mit gleicher Energiemenge (25 M.K.S. in 10 Sek.) beleuchtet waren. A wurde nach der Reizung auf den intermittierenden Klinostaten gestellt, B aber in vertikalem Stande gelassen. Man sieht hier sehr schön den groszen Unterschied im Krümmungsverlaufe. Auch Rothert (1. c. fig. 12) hat Abbildungen von Pflanzen gegeben, welche sich auf dem Klinostaten krümmen. Diese sind aber einige Stunden durchbeleuchtet, während die hier abgebildeten Krümmungen ganz die Folge einer Nachwirkung sind. Miszt man bei auf dergleiche Weise bekommenen Abbildungen die Stärke der Krümmung verschiedener Zonen, dann zeigt sich, dasz nach ungefähr 6 Stunden die Krümmung der höheren Zonen stark abgenommen ist, während sie nach der Basis zu noch an Stärke zunimmt. Das Maximum in Fig. 2 kommt also dadurch zu stande, dasz in diesem Augenblicke die Geradestreckung der Spitzenregion gröszer geworden ist als der Zuwachs der Krümmung in den mehr basalen Zonen.

\section{§5. Reaktions- und Krümmungszeit.}

Der Anfang der phototropischen Krümmung wurde hier beschrieben als eine Symmetrieänderung der Spitze des Keimlings. Viele Untersucher haben groszen Wert gelegt auf den Augenblick, wo die Krümmung anfängt. Es musz also untersucht werden, ob es möglich ist den Augenblick $z \mathrm{u}$ bestimmen in dem die Spitzenasymmetrie entsteht. Es hat sich gezeigt, dasz das äuszerst schwer ist, da man wohl nach einer bestimmten Zeit sagen kann dasz eine Asymmetrie vorhanden ist, aber dadurch, dasz sie so allmählich anfängt, ist es experimentell nicht möglich den Augenblick worin die Krümmung beginnt zu bestimmen. 
Alle Untersucher haben diese Schwierigkeit mehr oder weniger empfunden und dadurch ist die Reaktionszeit bei vielen in einen üblen Geruch gekommen. In Hinsicht auf die experimentell unlösbare Schwierigkeit ist es hier vorgezogen worden das Wort Reaktionszeit so anzuwenden, dasz es nicht nur ein theoretischer Begriff sondern auch eine experimentell $z \mathrm{u}$ bestimmende Grösze ist. Wenn man die Zeit, welche zwischen dem Anfange der Reizung und dem Augenblicke worin eine bestimmte Krümmungsstärke erreicht wird, verläuft, Krümmungszeit nennt, wird man unter experimentelle Reaktionszeit die Krümmungszeit $z u$ verstehen haben, welche $z u$ der kleinsten makroskopisch meszbaren Krümmung gehört. Die se experimentelle Reaktionszeit wird jedenfalls gröszer sein als die theoretische, welche durch das erste Auftreten der Krümmung bestimmt wird.

§6. Abhängigkeit der maximalen $\mathrm{Krümmung}$ von der Stärke des Reizes.

Die Kurven in Fig. 1 weisen schon darauf hin, dasz die Stärke der Krümmung nicht immer dieselbe ist, sie suggerieren schon die Voraussetzung, dasz die Stärke der Krümmung eine Funktion der Energiemenge ist womit man gereizt hat. Mit dem Nachprüfen dieser Hypothese sind viele Beschwerden verbunden. Man würde eine sehr grosze Anzahl Versuche brauchen sie zu beweisen, da die Energiemenge beliebig vergröszert und durch Aenderung ihrer Komponenten unendlich variiert werden kann. Eine Lösung dieses äuszerst wichtigen Problemes kann hier also gar nicht erwartet werden. Nur soviel wie nötig ist um über die wichtigsten Punkte einigermaszen orientiert $z u$ sein ist hier untersucht worden.

Es ist hier erst eine Serie Versuche zu besprechen, welche eine Uebers bichtüer die Resultate von Reizungen 
mit ziemlich kleinen in kurzer Zeit zugeführten Energiemengen (1-100 M.K.S.) geben. Die Erscheinungen sind bei diesen Versuchen ziemlich einfach. Dennoch war es auch hier sehr schwierig genügende Angaben zu bekommen:

$1^{0}$. indem eine einfache Weise den Krümmungsverlauf zuverlässig zu beschreiben fehlt;

$2^{0}$. durch die Variabilität im Krümmungsprozesse; nicht nur haben Pflanzen verschiedener Länge einen anderen Krümmungsverlauf und ist auch das Alter von Einflusz, sondern dazu kommen noch die vielfachen Nutationen und individuellen Abweichungen. Da diese Versuchsserie im Dunkelzimmer des Laboratoriums ausgeführt worden ist, wo die Temperatur nicht reguliert werden kann, verstärken die bei verschiedenen Versuchen abwechselnden Temperaturen (gröszte Differenz $2,5^{\circ}$ ) die Variabilität der Resultate.

Um eine möglichst grosze Anzahl Wahrnehmungen zu erzielen, ist eine Aufstellung benutzt worden wobei der Krümmungsverlauf verschiedener Pflanzen zu gleicher Zeit verfolgt werden kann. Das Bild der Keimlinge wird durch eine Linse unter zweimaliger Vergröszerung auf eine gläserne Platte projiziert, worauf photographisch eine Teilung in Rauten angebracht ist. Mit einer Lupe kann die Lage der Spitzen abgelesen werden. Auf diese Weise ist es möglich die horizontale Spitzenabweichung von 5 bis 8 Pflanzen zu gleicher Zeit zu bestimmen.

Von den vielen Versuchen, welche angestellt worden sind, wird eine grosze Anzahl hier nicht mitgeteilt werden, da bei diesen entweder die Temperatur zu viel von der Durchschnittstemperatur abwich oder Nutationen schon vor dem Anfange der phototropischen Krümmung auftraten. Als fester Punkt im Krümmungsprozesse ist hier die Stärke der horizontalen Abweichung gewählt, im Augenblicke wo die Schwerkraft die Zunahme der phototropischen Krümmung aufhören läszt, d. h. die phototropische Krüm- 
mung ihre maximale Stärke erreicht. Die verschiedenen Mengen Lichtenergie sind durch Beleuchtung während derselben Zeit mit verschiedener Intensität hergestellt worden. Die Reizdauer beträgt bei all diesen Versuchen 10 Sekunden. Als Lichtquelle wurde invertes Gasglühlicht benutzt, welches auszerhalb des Dunkelzimmers aufgestellt war.

Tabelle 1.

\begin{tabular}{|c|c|c|c|}
\hline $\begin{array}{c}\text { Lichtmenge in } \\
\text { M.K.S. }\end{array}$ & $\begin{array}{l}\text { Grösze der } \\
\text { Maximal- } \\
\text { Krümmung bei } \\
\text { Länge von } \\
\pm 25 \mathrm{mM} \text {. }\end{array}$ & $\begin{array}{l}\text { Grösze der } \\
\text { Maximal- } \\
\text { Krümmung bei } \\
\text { Länge von } \\
\pm 20 \mathrm{mM} \text {. }\end{array}$ & $\begin{array}{l}\text { Grösze der nach } \\
2 \text { Stunden } \\
\text { erreichten Krüm- } \\
\text { mung bei Länge } \\
\text { von } \pm 25 \text { mM. }\end{array}$ \\
\hline
\end{tabular}

\begin{tabular}{r|c|c|c}
7.6 & $0,7(21)$ & $0,5(5)$ & $0,5(16)$ \\
12,4 & $1,-(12)$ & - & - \\
18,1 & $1,6(16)$ & - & $1,2(11)$ \\
26,4 & $2,3(22)$ & $2,-(16)$ & - \\
45 & $3,-(38)$ & - & $2,-(23)$ \\
65 & $3,3(29)$ & $2,6(17)$ & - \\
75 & $4,-(14)$ & - & - \\
100 & $5,-(31)$ & $3.1(9)$ & $3,5(24)$
\end{tabular}

Hinter den Zahlen, welche die Grösze der maximalen Krümmung angeben, ist zwischen Klammern die Anzahl der Pflanzen angegeben worden woraus dieser Wert berechnet worden ist. Mittlere Temperatur $17,5^{\circ} \mathrm{C}$. Pflanzen nicht bei konstanter Temperatur gezogen.

Aus den Werten dieser Tabelle kann man zwei Folgerungen ziehen.

$1^{0}$. Zu jeder Stärke des Reizes gehört a uch eine Krümmung bestimmter Stärke.

$2^{0}$. Je gröszer die Energiemenge ist womit gereizt worden ist, destomehrnimmt a uch die maximale Krümmung $z u$. Die Tabelle zeigt dasz 
von 7,6 M.K.S. bis 100 M.K.S. die Krümmungsstärke von 0,7 bis $5 \mathrm{mM}$. zunimmt.

In diese Tabelle sind auch einige Werte für Pflanzen kleiner als $20 \mathrm{mM}$. aufgenommen worden. Es geht aus ihnen hervor, dasz die maximale horizontale Abweichung bei kleineren Pflanzen einen geringeren Wert erreicht.

Die kleinste Energiemenge, welche in diese Tabelle aufgenommen worden ist, beträgt 7,6 M.K.S. Die angewandte Methode läszt die Messung einer kleineren Krümmung nicht zu. Auch jetzt kann man mit Hilfe eines Mikroskopes kleinere Krümmungen untersuchen. So ist es gelungen bei 1,4 M.K.S. eine $\mathrm{Max}$ imalkrümmung von $1 / 4 \mathrm{mM}$. festzustellen. $\mathrm{D}$ a a ber a uf e inzelne Bestimmungen doch nicht ein groszer Wert gelegt werden kann, ist es wichtiger mit ziniger Uebung auch ohne Hilfe des Mikroskopes solche schwache Asymmetrieen beobachten zu können.

Die günstigsten Bedingungen diese Erscheinungen zu beobachten werden hier kurz zusammengefaszt werden da man von verschiedenen Seiten bezweifelt hat dasz so kleine maximale Krümmungen vorkämen. Man erreicht eine günstige Aufstellung, wenn man über eine Distanz von ungefähr $50 \mathrm{~cm}$. bis auf 3 Meter von der Lichtquelle eine Reihe von Pflanzen aufstellt. Vor dem Anfange des Versuches müssen alle Pflanzen, welche nicht gerade stehen und auszerdem alle deren Spitze nicht ganz symmetrisch ist, entfernt werden. Man wählt die Lichtmengen so, dasz in einer Distanz von 3 Meter ungefähr 1 M.K.S. zugeführt wird. Eine Temperatur von $16^{\circ}$ oder $17^{\circ} \mathrm{C}$, ist für diese Versuche zweckmäszig, da sich dann störende Einflüsse weniger gelten lassen. Als Lichtquelle die Krümmung $z u$ beobachten ist eine elektrische Glühlampe in einer Sachsschen Glocke mit Saffraninlösung zu empfehlen. Das Licht ist durch Pergamentpapier oder dergleichen diffus 
zu machen. Hält man nun von Zeit zu Zeit gegen diesen Hintergrund die gereizten Keimlinge, dann sieht man nach einiger Uebung eine Spitzenasymmetrie als Anfangsstadium der Krümmung zuerst bei den Pflanzen auftreten, welche das meiste Licht empfangen haben. Allmählich geht diese Asymmetrie in eine starke Krümmung über. Je weiter eine Pflanze sich von der Lichtquelle befindet, desto länger wird es dauern bevor die Asymmetrie auftritt und desto schwächer wird ihre maximale Stärke sein. Nimmt man 2 Stunden nach dem Anfange der Reizung wahr, dann gibt es ein äuszerst allmähliger Uebergang von der schwächsten Spitzenasymmetrie zu deutlich sichtbaren Krümmungen. Sind bei der Beleuchtung alle Pflanzen ordentlich gereizt worden, so wird die Reihe der gekrümmten vollkommen regelmäszig sein und ist es ganz unmöglich eine Stelle zu bestimmen wo die Pflanzen nicht gekrümmt sind. Je mehr Sorge man auf das Ziehen der Keimlinge verwendet, desto regelınäsziger und schöner zeigt sich diese Erscheinung.

$\S 7$. Krümmungszeit der Maximalkrümmung.

In Tabelle 2 sind einige Werte zusammengestellt worden, welche eine Uebersicht über die Zeit geben, welche verläuft bis die Maximalkrümmung erreicht wird. Bei starken Krümmungen ist die maximale Krümmungszeit stark variabel.

$$
\begin{aligned}
& \text { Tabelle } 2 . \\
8 & \text { M.K.S. } \\
18 & \text { M.K.S. } \\
26 & \text { M.K.S. } \\
45-100 & \text { M.K.S. }
\end{aligned}
$$

145 Min.
170 Min.
190 Min.
\pm 230 Min.

Die Werte dieser Tabelle haben ein praktisches Interesse in soweit sie angeben innerhalb welcher Zeit man wahrnehmen musz. Zugleich geht aus ihnen hervor, dasz 
eine starke Krümmung während langer Zeit fähig bleibt, die durch die Schwerkraft induzierte Gegenkrümmung zu überwinden.

$\S$ 8. Die Stärke der Krümmung nach einer bestimmten Zeit.

Die Werte, welche für die Maximalkrümmung bei bestimmter Energiemenge erhalten sind, sind zwar innerhalb gewisser hier nicht näher bestimmter Grenzen konstant, aber es ist doch erwünscht einen Maszstab für die Krümmungsstärke $\mathrm{zu}$ haben, welcher einfacher $z$ li bestimmen ist. Auszerdem ist die Maximalkrümmung die Resultante zweier Reizprozesse: der phototropischen Krümmung und der geotropischen Gegenreaktion.

Es wäre also besser die Stärke der Krümmung zu bestimmen in einem Augenblicke wo die geotropische Gegenreaktion noch keine so grosze Störung verursacht hat. Die Maximalkrümmung ist nur deshalb so konstant für einen gleichen Reiz weil die ganze Krümmung immer auf dieselbe Weise verläuft.

Die Stärke der Reaktion kann man also bestimmen auszer durch die Maximalkrümmung auch durch die Krümmungsstärke, welche in einem gewissen Augenblicke erreicht ist.

In Tabelle 1 sind einige Werte für die Stärke der Krümmung nach 2 Stunden zusammengestellt worden.

Am genauestem wird diese Methode sein wenn durch Rotation um die horizontale Achse eines Klinostaten die Gegenwirkung der Schwerkraft ganz aufgehoben wird.

§9. Gleichartige und ungleichart ge $\mathrm{K} r$ üm mungen.

In der im vorigen Paragraphen mitgeteilten Weise ist bei vielen hiernach beschriebenen Versuchen die Stärke 
der Reaktion gemessen worden. Experimentell ist diese Methode sehr leicht auszuführen. Mit ihrer Anwendung ist aber die Bedingung verbunden, dasz auf diese Weise nur gleichartige Krümmungen verglichen werden dürfen. Es ist schwer eine Definition $z u$ geben was man unter gleichartige Krümmungen $z \mathrm{u}$ verstehen hat. Es ist leicht einzusehen, dasz der Verlauf einer Krümmung sich gänzlich durch alle Umstände welche den Krümmungsprozesz beeinflussen ändert. (z. B. Temperatur, verdünnte Luft.) Wichtig ist, dasz auch die Zeit der Induktion insbesondere wenn sie länger ist als die Reaktionszeit einen Einflusz auf die Geschwindigkeit, womit die Krümmung stattfindet haben musz. Unter gleichartigen Krümmungen wird man also jene $z u$ verstehen haben welche verursacht werden durch Reize, welche in gleicher Zeit zugeführt sind und wobei die äuszeren und inneren Umstände so viel wie möglich dieselben sind. Es wird sich zeigen, dasz es in vielen Fällen unerläszlich ist ungleichartige Krümmungen $z u$ vergleichen. Schon hier darf darauf hingewiesen werden dasz man dabei vor unüberwindliche Beschwerden zu stehen kommt. Erst wenn es möglich sein wird mathematisch die Summation induzierter Krümmungsneigungen zu berechnen, werden ungleichartige Krümmungen verglichen werden können. Die Schwierigkeit ungleichartige Krümmungen $z u$ vergleichen haben viele Untersucher gefühlt. Hierdurch ist man auch dazu gekommen so groszen Wert auf die Schwellenbestimmungen zu legen. Hätte man wirklich mit einer Reizschwelle gearbeitet so würde man die Schwierigkeit mit ungleichartigen Krümmungen zu arbeiten überwunden haben. Es hat sich aber gezeigt dasz man nicht mit einer wirklichen Schwelle gearbeitet hat. Das Bestreben so viel wie möglich schwache ungleichartige Krümmungen zu vergleichen hat die Schwierigkeiten durchaus nicht gelöst sondern sie in ein Gebiet versetzt, wo die relativen Differenzen weniger leicht beobachtet werden können. 
§10. Abhängigkeit der Krümmungsstärke von der Energie bei nicht durch Schwer-

kraftwirkung beeinfluszten

Kr üm m ungen.

Ueber die Frage, wie der Verlauf einer geotropischen Krümmung abhängig von der Stärke des Reizes sei, wenn die Pflanzen nach der Beleuchtung der einseitigen Wirkung der Schwerkraft entzogen seien, können hier nur einige oriëntierende Versuche mitgeteilt werden. Es ist nicht überflüssig darauf hinzuweisen, dasz insbesondere von dergleichen Bestimmungen eine Lösung der oben besprochenen Problemen erhofft werden kann. Unerläszlich für diese Versuche ist ein zuverlässiger Klinostat.

Neben dem Pfefferschen Klinostaten, womit alle Versuche in Utrecht angestellt worden sind, habe ich während eines Aufenthaltes im Groninger Botanischen Institut, die Gelegenheit benutzt einige Bestimmungen mit dem van Harreveldschen ${ }^{1}$ ) Klinostate auszuführen. Dieser Klinostat ist als gewöhnlicher Klinostat vollkommen zuverlässig, als intermittierender hat er durch seine schwerere Konstruktion und genauere Zeitregulierung grosze Vorteile über den Fittingschen, aber er besitzt natürlich gleichfalls die Beschwerden, welche notwendig mit dem Intermittieren verbunden sind, die einer möglichen Unregelmäszigkeit während des Ueberganges von einer Lage in die andere. Diese Beschwerden sind aber bei gleichmäsziger Verteilung des Gewichtes um die Achse des Klinostaten nicht sehr grosz.

Es ist die Frage ob auch bei phototropischen Krüm-

1) Van Harreveld, Ph. Ein Universal-klinostat. Recueil d. Trav. Bot. Néerl. Vol. IX, 1912. 
mungen, wobei der Einflusz der einseitig wirkenden Schwerkraft aufgehoben ist ein Unterschied zwischen schwachen und stärkeren gemacht werden kann und worin sie sich unterscheiden. Einige in der in $\$ 4$ beschriebenen Weise verfertigten Zeichnungen machen es möglich auf diese Frage eine vorläufige Antwort zu geben. Diese Beobachtungen beziehen sich auf Reizungen bei Energiemengen von 13,2 M.K.S. (Fig. 4), 25 M.K.S.(Fig.5), 120 M.K.S.(Fig. 6), vergl. dazu noch Fig. 11 und 12 bei 7,25 M.K.S.(Temp. $25^{\circ}$ C.).) Es zeigt sich überzeugend, dasz auch jetzt die stärkere Reizung eine stärkere Krümmung ver ursacht. Bei der schwachen Reizung von 13,2 M.K.S. erreicht die Krümmung bald ein Maximum da sich hier nur ein kleiner Teil der Pflanze krümmt, und

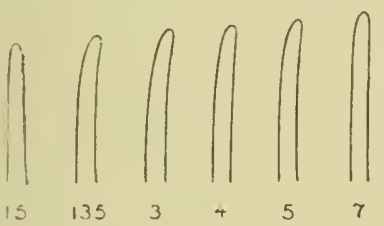

Fig. 4.

Positiv phototropische Krümmung. Reizstärke 13,2 M.K.S. (in 2 Sek.) $17,5^{\circ}$ C. Nach der Beleuchtung auf dem intermittierenden Klinostaten.

Die zwei ersten Zahlen geben die Zeit an nach dem Anfang der Reizung in Minuten, die übrigen in Stunden.

dieser sich schon nach wenigen Stunden gerade streckt. Bei einer starken Krümmung erreicht aber jede Zone e in Maximum; die oberen Zonen fangen zuerst an sich zu krümmen und strecken sich auch zuerst gerade. Nur wenn die Krümmung in den nicht mehr wachsenden Teil der Basis gekommen ist, wird sie fixiert. Die ganze Pflanze ist dann auszer dem Knick in der Basis vollkommen gerade. Es ist aber auszerordentlich schwierig für eine bestimmte Zone festzustellen, wann die Geradestreckung anfängt. Ein autotropische Bewegung über den Nullstand hinaus ist bei diesen Versuchen nie bemerkt worden; die Beobachtungen welche hierüber in der $\mathrm{Li}$ teratur $z u$ finden sind, müssen auf andere Weise interpretiert werden. 


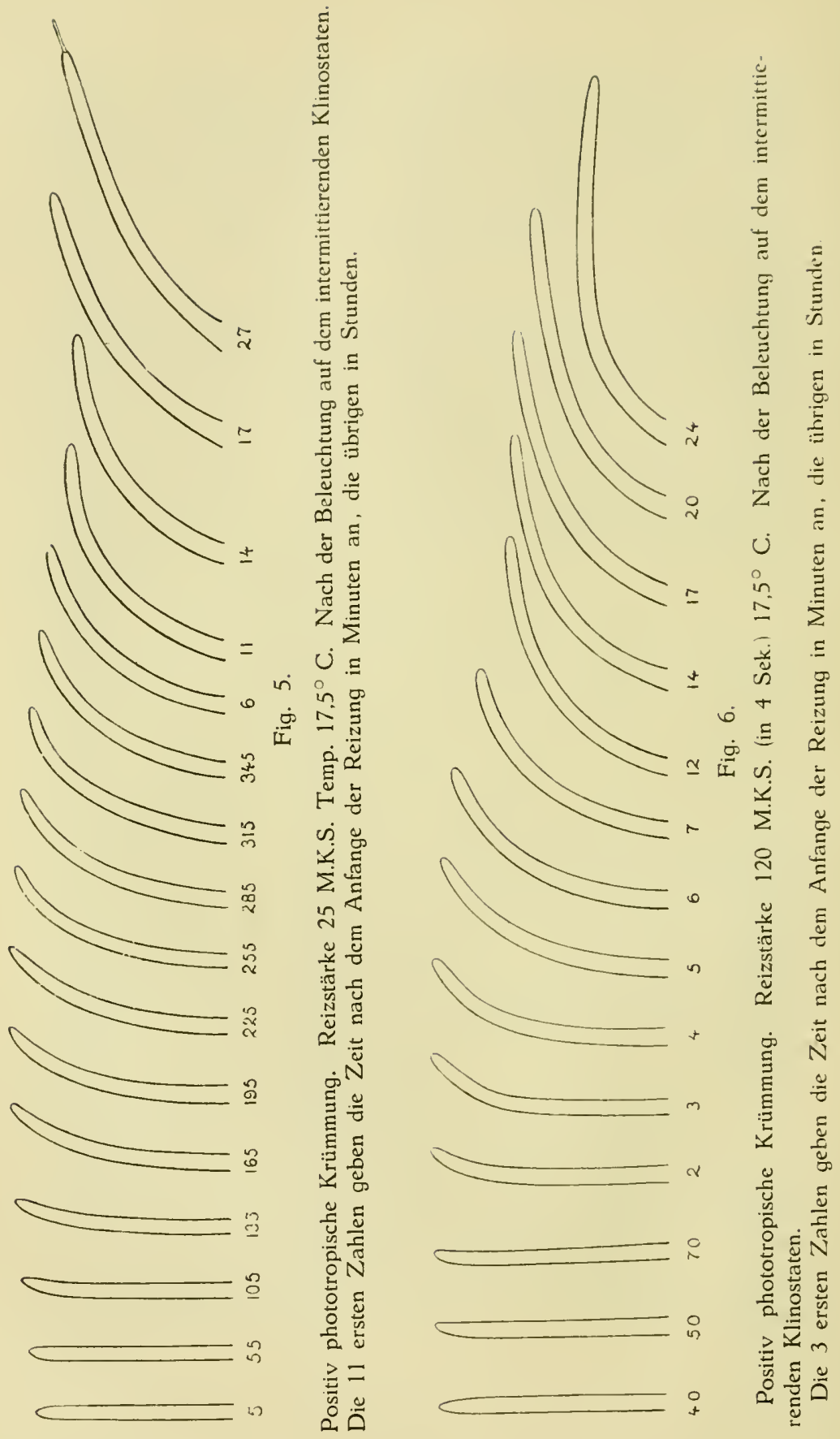


$\S 11$. Abhängigkeit der experimentellen Reaktionszeit von der Reizstärke.

In $\S 5$ wurde die experimentelle Reaktionszeit definiert als die Krümmungszeit, welche zu einer eben sichtbaren makroskopischen Krümmung gehört.

Hier ist zu untersuchen ob die Reaktionszeit ähnlich wie die Stärke der Krümmung von der Reizstärke abhängig ist. Auch dieses Problem ist $z u$ ausführlich um hier ganz untersucht zu werden. Die Beobachtungen beschränken sich auf den einfachsten Fall dasz die Energie womit gereizt wird in kurzer Zeit zugeführt wird, während in diesem $\S$ nur Energiemengen von 1-100 M.K.S. besprochen werden. Die Genauigkeit, womit die Reaktionszeit

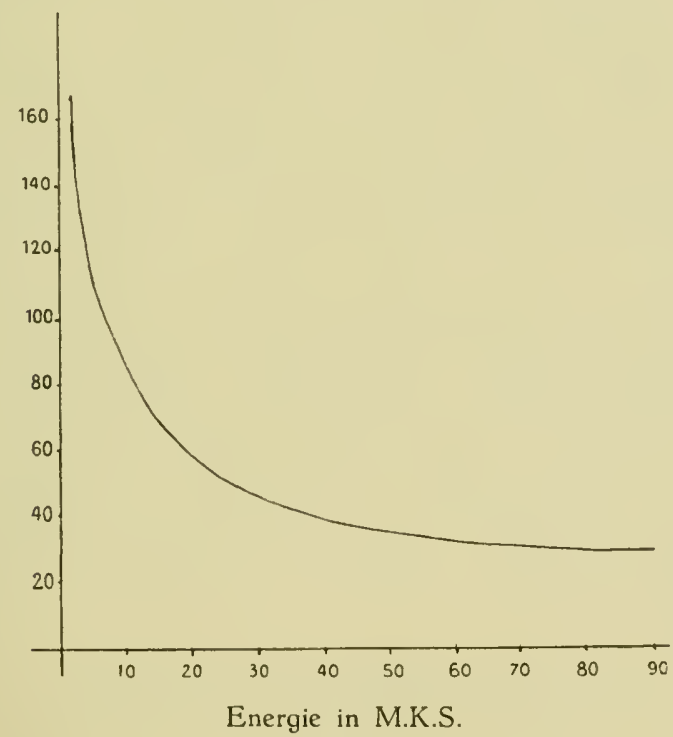

Fig. 7.

Beziehung zwischen Energie und der Zeit, bis die Krümmung eben makroskopisch beobachtet werden kann. Temp. $17,5^{\circ} \mathrm{C}$. Zeit auf dem Ordinat in Minuten. 
bestimmt werden kann, ist bei starken Krümmungen ziemlich grosz, bei äuszerst schwachen Krümmungen, wo die Asymmetrie ganz leise zunimmt, viel geringer. Die hier mitgeteilten Werte für die Reaktionszeit beanspruchen keine grosze Genauigkeit und haben nur den Zweck orientierende Uebersicht zu geben. Fig. 7 gibt ein Bild der starken Zunahme der Reaktionszeit bei Abnahme der Reizstärke.

Bei der sehr schwachen Energiemenge von 5 M.K.S. tritt die Spitzenasymmetrie erst nach ungefähr 2 Stunden auf. Bei 50 M.K.S. beträgt die Reaktionszeit nur 30 Minuten und erreicht damit ihren kleinsten Wert.

Obwohl in $\S 6$ gefunden wurde, dasz die Krümmungsstärke auch bei Reizen von 50-100 M.K.S. zunimmt, ist doch die kleinste Reaktionszeit schon bei 50 M.K.S. erreicht. Nur bei Reizungen zwischen 1 und 50 M.K.S. würde es also möglich sein, die Reaktionszeit als Masz für die Stärke der Reizung zu benutzen. Um zuverlässige Werte für die Reaktionszeit zu erhalten, wird man nicht den Augenblick bestimmen müssen, worauf die Reaktion eben sichtbar wird, da dieser sehr von der individuellen Variabilität des Beobachters und der Pflanze abhängt, sondern den Augenblick wo eine Krümmung einer bestimmten meszbaren Stärke erreicht wird. Diese Methode ist das Analogon der in $\S 8$ beschriebenen Bestimmung der nach einer bestimmten Zeit erreichten Krümmungsstärke. Sie ist aber experimentell viel schwieriger auszuführen und ist dadurch weniger geeignet die Stärke der Reaktion zu messen.

\section{$\S 12$. Schwellenbestimmung.}

Aus dem Gesagten geht hervor, dasz der Effekt einer Beleuchtung geringer ist je nachdem die zugeführte Energiemenge auch geringer ist. Ein schwächerer Effekt äuszert 
sich in einem später Sichtbar werden der Krümmung (längere Reaktionszeit) und im Erreichen einer geringeren maximalen Stärke. Die Frage ist jetzt ob man experimentell einen Schwellenwert für die Stärke des Reizes feststellen kann, worunter gar keine Krümmungen auftreten. In $\S 6$ ist darauf hingewiesen, dasz je nachdem mehr Sorge und Akkuratesse auf die Experimente verwendet wird der Uebergang von starken in schwache Krümmungen allmähliger stattfindet. Man braucht sich also auch nicht zu wundern, dasz es ganz unmöglich ist eine Energiemenge anzugeben, wobei die Krümmung plötzlich unsichtbar wird. Eine scharfe Grenze besteht nicht und ist nur künstlich zu ziehen. In diesem Augenblicke musz es für experimentell unmöglich gehalten werden einen Schwellenwert für das Auftreten der Reaktion festzustellen. Ganz unabhängig von dieser experimentellen Schwierigkeit ist die Frage, welche später noch $z u$ berücksichtigen ist, ob es theoretisch notwendig sei, dasz eine Energieschwelle bestehe. Experimentell ist es nur gestattet mit einer Krïmmung von bestimmter Stärke zu arbeiten. Die Bestimmung des wirklichen Anfangs der sichtbaren Reaktion (theoretische Reaktionszeit) und des Schwellenwertes des Reizes, welcher eben noch eine Krümmung veranläszt, sind zwei analoge Fälle, welche in diesem Augenblicke als experimentell unlösbar betrachtet werden müssen. 


\section{ABSCHNITT II. \\ Die negative Reaktion.}

\section{$\S$ 13. Die negative Reaktion.}

In Tabelle 3 sind die Werte zusammengestellt worden welche für die maximale Krümmungsstärke bei gröszeren Lichtmengen als 100 M.K.S. gefunden worden sind. Auch bei diesen Versuchen war der Reizdauer 10 Sek. und die mittlere Temperatur $17,5^{\circ}$ C. so dasz diese Tabelle sich der Tabelle 1 anschlieszt.

TABELLE 3.

\begin{tabular}{ccc}
$\begin{array}{c}\text { Lichtenergie } \\
\text { in }\end{array}$ & $\begin{array}{c}\text { Stärke der } \\
\text { max. Kr. in mM. } \\
\text { (Anzahl Planzen.) }\end{array}$ & $\begin{array}{c}\text { Zeit bis die } \\
\text { max. Kr. } \\
\text { erreicht ist. }\end{array}$ \\
\hline 100 & 5 & $(31)$ \\
140 & 4,7 & $(26)$ \\
237 & 5,4 & $(24)$ \\
560 & $4,-$ & $(31)$ \\
1500 & $3,-$ & $(49)$ \\
2800 & 1,2 & $(82)$
\end{tabular} \mid \pm 230 Min.

Aus den hier für die maximale Krümmung gefundenen Werten geht hervor, dasz von $100-137$ M.K.S. die Krümmung ungef́ähr gleich stark bleibt, dasz sie aber bei noch stärkerer Energie 
schwächer wird. In der letzten Kolomne dieser Tabelle sind die Zeiten aufgenommen, wo die maximale Krümmung erreicht wird. Aus den Zahlen kann man sehen, dasz dies bei gröszeren Energiemengen früher stattfindet.
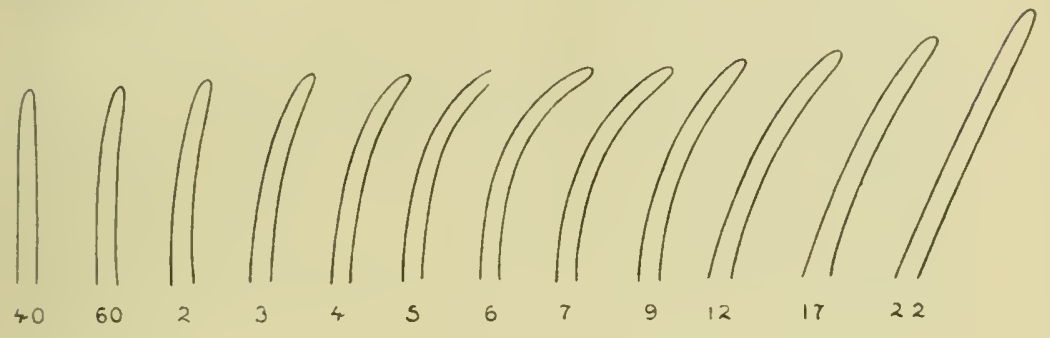

Fig. 8.

Positiv phototropische Krümmung. Reizstärke 1800 M.K.S. (in 60 Sek.) $17,5^{\circ}$ C.

Nach der Beleuchtung auf dem intermittierenden Klinostaten.

Die 3 ersten Zahlen geben die Zeit nach dem Anfang der Reizung in Minuten an, die übrigen in Stunden.

Fig. 8 gibt eine Abbildung einer derartigen Krümmung, wenn diese sich ungestört auf dem Klinostaten äuszern kann. Diese Pflanze war mit 1800 M.K.S. bei $18^{\circ} \mathrm{C}$. gereizt worden und zeigt bei Vergleichung mit der in Fig. 6 (p. 69) abgebildeten Pflanze eine ansehnlich schwächere Krümmung, insbesondere in den späteren Krümmungsstadien.

Wird mit noch stärkerer Energie gereizt, so nimmt die positive Krümmung noch mehr ab und tritt das Zurückgehen der Krümmung früher auf. Dieses Zurückgehen der Krümming hat eine nähere Untersuchung bedürft, da diese Gegenreaktion sehr stark den Eindruck gab zu einer negativ phototropischen Krümmung zu führen.

Es stand vom Anfange an fest, dasz um diese Erscheinungen zu analysieren es absolut notwendig war die Induktion von Gegenkrümmungen durch 
die Schwe r k raf t durch Rotation auf einem Klinostaten um horizontale Achse auszuschlieszen. Ein Teil dieser Versuche ist im Botanischen Institute in Groningen mit dem van $\mathrm{H}$ arreveldschen Klinostaten ausgeführt worden, in einem Zimmer für konstante Temperatur bei $25^{\circ} \mathrm{C}$.

Bei diesen Versuchen ist nur eine Spitzenzone von $2 \mathrm{mM}$. oder weniger beleuchtet worden da aus Untersuchungen von $\mathrm{V}$ an der Wolk hervorgegangen war, dasz.bei Beleuchtungen von mehr als 2000 M.K.S. in der Basis induzierte Krümmungen zu erwarten sind. Obwohl es sich bei näherer Untersuchung (v. Abschnitt III § 21) gezeigt hat, dasz diese Angaben ähnlich wie diejenigen Wilschkes nicht ganz richtig sind, so musz es doch als ein Vorteil betrachtet werden, dasz nur eine kleine Spitzenzone bei diesen Versuchen gereizt wurde.

In derselben Weise als in $\S 10$ schon für eine positive Krümmung beschrieben worden ist, sind auch vom Krümmungsverlaufe einer negativen Krümmung, welche auf
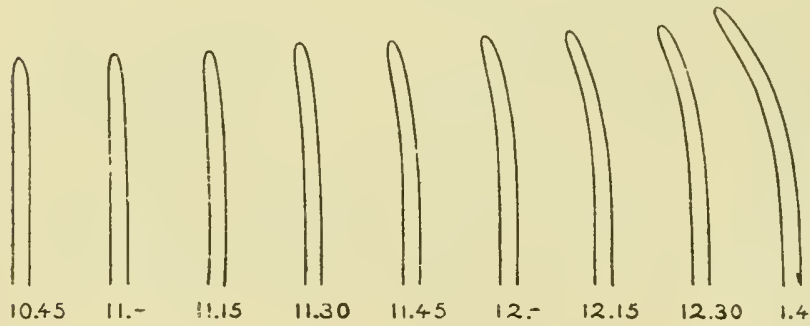

11.45

12.-

12.15

$12.30 \quad 1.45$

Fig. 9.

Negativ phototropische Krümmung eines Avenakeimlinges.

10,15 wurde die Spitzenzone $(2 \mathrm{mM}$.) während 40 Sekunden mit eirer Intensität von 340 M.K. von rechts beleuchtet. Nach der Beleuchtung auf dem intermittierenden Klinostaten. Temp. $25^{\circ}$ Cels. Der Pfeil gibt die Lichtrichtung an.

dem Klinostaten ungestört stattfindet, Zeichnungen verfertigt worden.

Fig. 9 gibt den Verlauf einer rein negativen Krüm- 
mung. Diese wurde durch eine Beleuchtung einer $2 \mathrm{mM}$. langen Spitzenzone mit einer Intensität von 340 M.K.S. während 40 Sek. bei $25^{\circ}$ C. verursacht. Nach der Beleuchtung wurde das Pflänzchen an die horizontalen Achse des intermittierenden Klinostaten befestigt und mittels des Projektionszeichenapparates bei rotem Lichte die hier reproduzierten Zeichnungen verfertigt.

Nach 45 Minuten ist die Krümming schon deutlich sichtbar. Die Reaktionszeit für diese negative Krümmung ist also sicher kleiner als 45 Minuten. Genau so wie bei einer positiven Krümmung allmählich nicht beleuchtete Zonen an der Krümmung teilnehmen, breitet sich auch die negative Reaktion von der allein beleuchteten Spitzenzone nach den mehr basal gelegenen Zonen aus. Auch bei einer negativen Krümmung findet also Reizleitung statt.

Wird mit weniger Energie gereizt z. B. während 30 Sek. mit einer Intensität von 340 M.K., dann ist die negative Reaktion schwächer. Eine schwache positive Krümmung der Spitze geht ihr dann voraus.

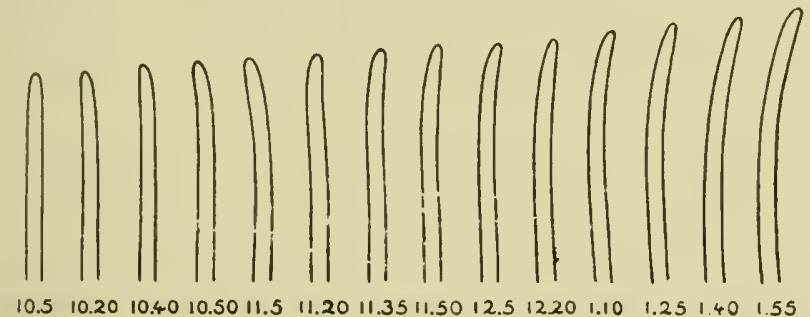

Fig. 10.

Positiv und negativ phototropische Krümmung eines Avenakeimlinges.

9,50 wurde eine Spitzenzone kleiner als $1 \mathrm{mM}$. während 30 Sekunden mit einer Intensität von 340 M.K. von links beleuchtet. Bis 11.5 eine positive Krümmung, später eine starke negative. Nach der $\mathrm{Be}$ leuchtung auf dem intermittierenden Klinostaten. Temp. $25^{\circ}$ Cels.

Wie man auf den in Fig. 10 reproduzierten Zeichnungen deutlich sehen kann fängt erst eine gewöhn- 
liche positive Krümmung an. Indem die Basis sich noch weiter positiv krümmt, beginnt die Spitze eine Krümmung in der gegenübergestellte Richtung auszuführen. Auch an dieser Krümmung nehmen allmählich mehr basal gelegenen Zonen teil und es bleibt zum Schlusz eine schwache aber deutliche negative Krümmung.

Die Erklärung dieser kombinierten negativen und positiven Krümmung bringt einige Schwierigkeiten mit. Man könnte es sich verständlich machen durch die Annahme dasz in der äuszersten Spitze, dem empfindlichsten Teil des Koleoptyles, eine negative Krümmung induziert wird. indem eine mehr basale Zone, welche weniger empfindlich ist, die positive Krümmung verursacht. Diese Er klärung ist aber weniger plausibel da auch bei einer Spitzenzone, welche kleiner ist als $1 \mathrm{mM}$. diese Erscheinung auftritt. (Vergl. § 41). Werden diese verwickelten Phänomene nicht so wie hier mit Hilfe eines Klinostaten untersucht, dann ist es nie möglich mit Sicherkeit zu bestimmen ob ein Zurückgehen der positiven Krümmung durch die Schwerkraft verursacht wird oder durch das Zusammenwirken der Schwerkraft mit negativer Krümmung.

§14. Die Energiemengen bei welchen eine negative Krümmung auftritt.

Um eine Idee $z u$ bekommen von welchen Umständen das Zustandekommen negativer Krümmungen abhängt, ist es erwünscht den Effekt verschiedener in verschiedenen Zeiten zugeführten Energiemengen systematisch zu untersuchen.

Die grosze Anzahl Versuche, welche schon für eine vorläufige Orientierung erfordert werden, hat es unmög lich gemacht diese Erscheinungen in Detail zu untersuchen. Nur die Reaktionsweise wurde bestimmt. 
In den Tabellen 4-11 sind die Beobachtungen zusammengestellt worden, welche sich auf das Gebiet wo negative Krümmungen vorkommen beziehen, nach den Intensitäten womit gereizt worden ist, geordnet. Für die Bestimmung jedes Wertes sind wenigstens 20 Pflanzen benutzt worden. meistens aber viel mehr. Die wichtigen Punkte sind mehrere Male und unter verschiedenen Umständen untersucht. Die Genauigkeit der bestimmten Zahlen ist unter den gegebenen Umständen hauptsächich von der Konstanz der Lichtquelle abhängig: der hierdurch verursachte Fehler ist sicher nicht weniger als $10 \%$.

$$
\text { Tabelle } 4 .
$$

Intensität 1.4 M.K.

Reizdauer.

10 Min.

15 Min.

20 Min.

24 Min.

26 Min.

30 Min.

Energie.

840 M.K.S. +

1260 M.K.S. + schwache geotr. Aufr.

1680 M.K.S.

2016 M.K.S.

2184 M.K.S,

2520 M.K.S.

Tabelle 5 .

Intensität 5,5 M.K.

Reizdauer.

Energie.

6 Min. 1980 M.K.S. + schwache geotr. Aufr.

10 Min. 3300 M.K.S.

14 Min. 4620 M.K.S.

15 Min. 4950 M.K.S.

+ später - (schwach)

20 Min. 6600 M.K.S.

+ später - (schwach)

25 Min.

30 Min. 8250 M.K.S.

+ später - (schwach)

40 Min. 9900 M.K.S. 13200 M.K.S. 
Tabelle 6.

Intensität 12 M.K.

Reizdauer.

5 Min.

7 Min.

10 Min.

15 Min.

20 Min.

25 Min.
Energie. 3600 M.K.S. 5040 M.K.S. + später - (schwach) 7200 M.K.S. + später - (stark) 10800 M.K.S. + später - (stark) 14400 M.K.S. später - ?

$$
\text { Tabelle } 7 \text {. }
$$

Intensität 100 M.K.

Reizdauer.

$40 \mathrm{Sec}$.

$50 \mathrm{Sec}$.

$60 \mathrm{Sec}$.

$90 \mathrm{Sec}$.

5 Min.

7 Min.

10 Min.

11 Min.

13 Min.

15 Min.

20 Min.
Energie. 4000 M.K.S. 5000 M.K.S. - später 6000 M.K.S. 9000 M.K.S. 30000 M.K.S. 42000 M.K.S. 60000 M.K.S. 66000 M.K.S. 78000 M.K.S. 90000 M.K.S. 120000 M.K.S.

$$
\text { Tabelle } 8 .
$$

Intensität 450 M.K.

Reizdauer.

$5 \mathrm{Sec}$.

$10 \mathrm{Sec}$.

$30 \mathrm{Sec}$.

1 Min.

2 Min.

4 Min.

5 Min.
Energie. 2250 M.K.S. 4500 M.K.S. + später 13500 M.K.S 27000 M.K.S. 54000 M.K.S. 108000 M.K.S. - ? 135000 M.K.S. + ? oder 0 


$\begin{aligned} 6 \text { Min. } & 162000 \text { M.K.S. + ? } \\ 7 \text { Min. } & 189000 \text { M.K.S. + } \\ 10 \text { Min. } & 270000 \text { M.K.S. }+ \\ 20 \text { Min. } & 540000 \text { M.K.S. + } \\ 30 \text { Min. } & 810000 \text { M.K.S. }\end{aligned}$

$$
\text { Tabelle } 9 \text {. }
$$

\begin{tabular}{|c|c|c|}
\hline Reizdauer. & Energie. & \\
\hline $5 \mathrm{Sec}$ & 9000 M.K.S. & 一 \\
\hline $10 \mathrm{Sec}$. & 18000 M.K.S. & - \\
\hline $20 \mathrm{Sec}$. & 36000 M.K.S. & 0 \\
\hline $40 \mathrm{Sec}$. & 72000 M.K.S. & $+?$ \\
\hline $90 \mathrm{Sec}$. & 162000 M.K.S. & + (schwach) \\
\hline $150 \mathrm{Sec}$ & 270000 M.K.S. & + (schwach) \\
\hline 5 Min. & 540000 M.K.S. & + (schwach) \\
\hline $10 \mathrm{Min}$. & 1080000 M.K.S. & + (schwach) \\
\hline $20 \mathrm{Min}$. & 2160000 M.K.S. & + (schwach) \\
\hline
\end{tabular}

Tabelle 10.

\begin{tabular}{|c|c|c|}
\hline Reizdauer. & Energie. & \\
\hline 1 Sec. & 4600 M.K.S. & $-?$ \\
\hline $2 \mathrm{Sec}$. & 9200 M.K.S. & + ? später - \\
\hline $4 \mathrm{Sec}$. & 18400 M.K.S. & + (schwach) \\
\hline 8 Sec. & 36800 M.K.S. & + \\
\hline $16 \mathrm{Sec}$. & 73600 M.K.S. & + (stark) \\
\hline $30 \mathrm{Sec}$. & 138000 M.K.S. & + (stark) \\
\hline 4 Min. & 1104000 M.K.S. & + \\
\hline 15 Min. & 4140000 M.K.S. & $+? ?$ \\
\hline $60 \mathrm{Min}$. & 16560000 M.K.S. & $+?$ \\
\hline
\end{tabular}


Tabelle 11 .

\begin{tabular}{|c|c|c|}
\hline $\begin{array}{l}\text { Reizdauer. } \\
\pm \frac{1}{9} \mathrm{Sec} .\end{array}$ & $\begin{array}{c}\text { Energie. } \\
+\quad 10000 \mathrm{MKS}\end{array}$ & $-1 \mathrm{sc}$ \\
\hline $2 \mathrm{Sec}$ & 40000 M.K.S. & + \\
\hline $10 \mathrm{Sec}$. & 200000 M.K.S. & + \\
\hline $40 \mathrm{Sec}$. & 800000 M.K.S. & + (schwach) \\
\hline 4 Min. & 4800000 M.K.S. & 0 \\
\hline
\end{tabular}

Tabelle 9 von $\mathrm{Bla}$ a uw.

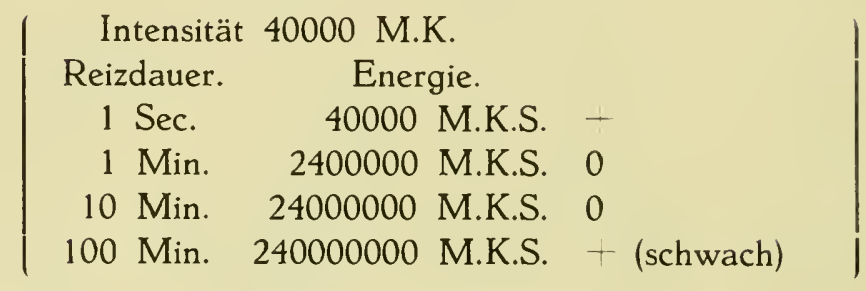

Wenn diese Versuche, ausgenommen diejenigen mit der höchsten Intensität sind bei $23^{\circ} \mathrm{C}$. im Zimmer für Konstante Temperatur im Laboratoriumsgewächshause angestellt worden (diejenigen mit den zwei höchsten Intensitäten im Vorlesungszimmer mit elektrischem Bogenlichte bei $20^{\circ} \mathrm{C}$.)

Immer sind die Pflanzen nach der Beleuchtung auf den Klinostaten gestellt worden, sodasz sie um die horizontale Achse drehend keine Gegenwirkung der Schwerkraft empfanden.

Wenn man die Resultate näher betrachtet, zeigt es sich dasz bei 1.4 M.K. (Tab. 4) nur ein ziemlich kleines Gebiet untersucht worden ist. Die Ursache ist, dasz es nicht möglich ist bei solchen schwachen Intensitäten wie 1,4 M.K. Versuche mit längerer Reizdauer auszuführen. Schon nach 30 Minuten sind die Spitzen deutlich positiv gekrümmt. Im Abschnitt $V$ wird die Erscheinung besprochen werden, dasz Licht das die Pflanzen unter einem kleineren Winkel 
als $90^{\circ}$ trifft eine andere Wirkung hat als Strahlen, welche die Pflanze senkrecht treffen. Wird also beleuchtet, indem die Spitze schon gekrümmt ist, so müssen die Ergebnisse unzuverlässig werden. Eine andere Beschwerde, dasz die Schwerkraft der Krümmung schon während der Beleuchtung entgegenarbeitet und dadurch eine geotropische Gegenkrümmung induzieren könnte, würde man noch überwinden können indem man die Pflanzen schon während der Beleuchtung auf dem Klinostaten um horizontale Achse drehen läszt. Die erste Beschwerde ist aber überwiegend gewesen um nicht länger als 30 Minuten zu beleuchten. In der Tabelle kann man sehen, dasz bis zu 30 Minuten Reizdauer keine negative Krümmung auftritt. Es wird noch auf andere Weise gezeigt werden, dasz es sehr unwahrscheinlich ist, dasz bei dieser schwachen Intensität auch bei längerer Beleuchtung negative Krümmungen auftreten sollten. (vergl. § 16)

Die in der Tabelle mitgeteilten geotropischen Aufrichtungen beziehen sich auf Beobachtungen, wobei die Pflanzen nicht auf den Klinostaten kamen sondern die Schwerkraft der Kriimmung entgegenwirkte. Wenn in den zwei ersten Stunden nach der Beleuchtung eine entgegengesetzt gerichtete Krümmung d.h. eine geotropische Aufrichtung sichtbar wird, ist das in dieser und der folgenden Tabelle angegeben worden.

Bei 5.5 M.K. (Tab. 5) tritt, wie lange auch beleuchtet wird, immer eine positive Krümmung auf. Bei sorgfältiger Untersuchung hat es sich aber gezeigt, dasz bei Reizungen von 14-20 Minuten nach der positiven Krümmung eine schwache negative sichtbar wird. Nur die Spitze krümmt sich schwach negativ, indem die Basis ganz positiv gekrümmt bleibt. Ein mit diesen Lichtmengen gereizter Keimling zeigt also die ersten zwei Stunden nach der Reizung eine normal verläufende positive Krümmung.

Nach ungefähr zwei Stunden wird dann die Spitze 
asymmetrisch, indem die ganze Basis ihre positive Krümmung behält. Diese negative Asymmetrie der Spitze ist $z u$ schwach um zu den basalen Zonen weitergeleitet zu werden. Eine dergleiche Krümmung ist auf Tafel I fig. I abgebildet worden, wo die Keimlinge 1 und 4 von rechts diese Erscheinung zeigen.

Bei der Intensität 12 M.K. (Tab. 6) sind die Erscheinungen dieselben als bei 5.5 M.K. Bei allen Beleuchtungszeiten eine positive Krümmung, indem in einem kleinen Gebiete nach der positiven eine negative Krümmung auftritt. Hier ist aber die negative Krümmung stärker als bei 5,5 M.K. und führt indem sie sich über mehr basale Zonen ausbreitet zu einer deutlichen S Form.

Bei allen stärkeren untersuchten Intensitäten ist es möglich eine rein negative Kr ümmung zu bekommen d.h. dasz keine makroskopisch wahrnehmbare positive Krümmung der negativen vorangeht.

Man kann hier also abhängig von der Dauer der Reizung, die folgenden Fälle unterscheiden: positive Krümmung; positive Krümmung welcher eine negative folgt; negative Krümmung (abgebildet in Fig. 9); positive Krümmung, welcher eine negative folgt; positive Kr ümmung.

Die Kombinationen von positiver und negativer Krümmung kommen abhängig von der Stärke des Reizes in allen Variationen vor.

Die Werte, welche für die 3 stärksten Intensitäten hier mitgeteilt sind, beanspruchen keine quantitative Genauigkeit. Sie dienen nur um zu zeigen, dasz bei den stärksten Lichtintensitäten sich gleichfalls die obengenannten Erscheinungen vorfinden. Zum Vergleich ist hier auch eine Tabelle aus Blaauw's Perzeption des Lichtes aufgenommen worden.

Es zeigt sich, dasz B la a u w zufälligerweise nicht die 
Energiemenge benutzt hat, welche eine negative Krümmung zu Folge hat. Die Ursache dessen ist, dasz bei diesen starken Lichtintensitäten das Gebiet wo negative Krümmungen auftreten äuszerst klein ist und $\mathrm{Blaauw}$ negative Krümmungen erst bei sehr groszen Energiemengen erwartete.

\section{§ 15. Die Gültigkeit der Produktregel für die negative Krümmung.}

Untersucht man bei welcher Energiemenge die negative Krümmung zuerst auftritt dann zeigt sich, dasz dies bei allen Intensitäten bei ungefähr demselben Wert von 4000 M.K.S. stattfindet. $\mathrm{Ob}$ bei 1,4 M.K. auch noch eine negative Krümmung aufgetreten wäre ist nicht mit Sicherkeit zu sagen; auf Grund der Ergebnisse von $\S 16$ nicht wahrscheinlich.

Für die negative Kr ümmung kann also die Regel festgestellt werden, dasz wenn die negative Krümmung auftritt, sie immer bei derselben Energiemenge aufzutreten anfängt. Ebenso wie für die positive Krümmung istauch hier die Produktregel gültig.

Eine starke Abweichung von den Zahlen, welche hier für den Anfang der negativen Krümmung mitgeteilt sind, zeigt eine Reihe von Beobachtungen welche $\mathrm{Clark}^{1}$ ) unabhängig von dieser Untersuchung publiziert hat.

In Tabelle 12 ist eine vergleichende Uebersicht der von ihm und mir gefundenen Werte gegeben worden.

Merkwürdig ist, dasz Clark bei schwachen Intensitäten schon bei kleinen Energiemengen negative Krümmungen auftreten sieht. Wenn dies richtig wäre, würde es für

1) Clark, O. L. Ueber negativen Phototropism us bei Avena sativa. Zeitschrift f. Botanik. Bd. 5. 1913. 
Tabelle 12.

Einseitige Beleuchtung.

Intensität in $\mathrm{M}$. K.

\begin{tabular}{|c|c|c|c|c|c|c|c|c|}
\hline & 1,4 & 5,5 & 12 & 100 & 450 & 1800 & 4600 & 20000 \\
\hline $\begin{array}{l}\text { Negative } \\
\text { Krümmung } \\
\text { beginnt bei }\end{array}$ & & \multicolumn{5}{|c|}{ \pm 4000 M.K.S. } & \multicolumn{2}{|c|}{$\begin{array}{l}\text { nicht bestimmt } \pm \\
\text { 10.000 M.K.S. neg }\end{array}$} \\
\hline $\begin{array}{l}\text { 2e Positive } \\
\text { Krümmung } \\
\text { beginnt bei }\end{array}$ & 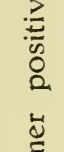 & 8250 & 18000 & 78000 & 135000 & \pm 72000 & \pm 18000 & \pm 20000 \\
\hline $\begin{array}{l}\text { Reizdauer } \\
\text { für 2e pos. } \\
\text { Krümmung }\end{array}$ & $\bar{E}$ & $25 \mathrm{Min}$ & $25 \mathrm{Min}$ & 13 Min. & 5 Min. & $40 \mathrm{Sec}$. & $4 \mathrm{Sec}$ & $1 \mathrm{Sec}$. \\
\hline
\end{tabular}

Einseitige Beleuchtung nach $\mathrm{Cla}$ rk.

$\begin{aligned} & \text { Neg. Krümmung } \\ & \text { beginnt bei }\end{aligned}$
$\begin{aligned} & \text { 2e pos. Kr. } \\ & \text { beginnt bei }\end{aligned}$
die Auffassung der negativen Krümmung und die Wirkung
groszer Energiemengen wichtig sein. Es können aber
wider die Beobachtungen Clark's Beschwerden angeführt
werden, infolgederen es besser ist keinen groszen Wert
auf seine Zahlen zu legen. Es ist mit grossem Nachdruck
betont worden, dasz nur dann zu einer negativen Krüm-
mung beschlossen werden kann wenn durch Rotation um
die horizontale Achse eines Klinostaten das Auftreten von
Gegenkrümmungen, welche durch die Schwerkraft induziert
sind, verhindert worden ist. Werden die Pflanzen nach


der Beleuchtung nicht auf den Klinostaten gestellt, so treten geotropische Gegenkrümmungen auf, welche den negativ phototropischen Krümmungen ähneln. In den Tabellen 4 und 5 ist aufgenommen worden bei welcher Reizdauer diese Gegenkrümmungen schon 2 Stunden nach dem Anfange der Beleuchtung sichtbar geworden sind.

In den Figuren 2 und 3 Tafel I sind Photographieen von Pflanzen gegeben worden, welche bei einer Intensität von 5 M.K. während 5 Minuten gereizt worden sind. Die Pflanzen, welche nicht auf dem Klinostaten waren, zeigen eine deutliche Gegenkrümmung. Die Kontrollepflanzen auf dem Klinostaten beweisen, dasz diese Gegenkrümmung keine phototropische ist, sondern dasz sie durch die Schwerkraft induziert wurde.

Stellt man also die Pflanzen nach der Beleuchtung nicht auf den Klinostaten so ist die Uebereinstimmung mit den von $\mathrm{Clark}$ mitgeteilten Werten merkwürdig viel gröszer. Wie Herr Prof. Jost so liebenswürdig war mitzuteilen hat $\mathrm{Clark}$ seine Pflanzen nach der Beleuchtung nie auf einem Klinostaten um horizontale Achse rotieren lassen. Man kann hier also fast mit Sicherheit seine abweichende Resultate seiner Methodik zuschreiben.

\$16. Das nicht Auftreten negativer Krümmungen bei schwachen Intensitäten.

Neben den Beobachtungen, welche auf oben beschriebene Weise verrichtet wurden, sind noch mit einer einigermaszen geänderten Aufstellung einzelne Versuchen ausgeführt worden um $z u$ versuchen festzustellen ob bei schivächeren Intensitäten als 5,5 M.K. eine negative Krümmung zu bekommen ist.

Es ist schon darauf hingewiesen worden, dasz es nicht möglich ist wegen des Auftretens der positiven Krümmung während der Dauer der Beleuchtung und der hierdurch 
geänderten Beleuchtungsumstände dieses Problem auf die bis jetzt gefolgte Weise zu lösen.

Als Lichtquelle ist für diese Versuche ein 10 Volt Lampe benutzt worden, welche mit konstant gehaltener Stromstärke auf einer Akkumulatorenbatterie brannte. Bei diesen Versuchen wurde jedesmal eine Reihe Keimlinge hinter einander aufgestellt und die Entfernung bis zur Lichtquelle bestimmt wobei eine negative Asymmetrie eben noch beobachtet werden konnte. Alle Pflanzen wurden gleich nach der Beleuchtung auf Pfeffersche Klinostaten gestellt um die geotropische Gegenkrümmung auszuschlieszen. Die auf diese Weise gefundenen Werte für das erste Auftreten der negativen Krümmung sind kleiner als diejenigen welche schon im vorigen Paragraphen mitgeteilt worden sind.

Bei der Beurteilung dieses Unterschiedes hat man damit Rechnung zu tragen, dasz bei dieser Aufstellung

$1^{0}$. die Zusammensetzung des Lichtes nicht dieselbe war,

$2^{0}$. die Intensität des Lichtes vollkommen konstant war. sodasz die hier gefundenen Werte einen geringeren Fehler haben,

3'. die Grenze wo noch eine Asymmetrie zu sehen ist auf diese Weise sorgfältiger bestimmt worden kann.

$4^{0}$. die Pflanzen bei konstanter Temperatur gezogen sind. (v. § 19)

Die Ergebnisse verschiedener Versuche zeigen eine genügende Uebereinstimmung.

$$
\text { Tabelle } 13 .
$$

Bestimmung des Anfanges der negativen Krümmung. Die Beleuchtungsstärke beträgt auf $1 \mathrm{M}$. Entfernung 5 M.K.

Reizdauer Negative Krümmung wird sichtbar

Energie

5 Min. bei $84,5 \mathrm{cM}$. von der Lichtquelle 2100 M.K.S.

10 Min. bei $125 \mathrm{cM}$. von der Lichtquelle 1920 M.K.S.

16 Min. bei $110 \mathrm{cM}$. von der Lichtquelle 2100 M.K.S.

25 Min. keine negative Krümmung, 
Bei Beleuchtungen von 5-16 Minuten tritt die negative Krümmung bei 2000 M.K.S. auf. Bei einer Beleuchtungsdauer von 25 Minuten wird aber gar keine negativ phototropische Krümmung sichtbar. Hiermit ist bewiesen, dasz bei Intensitäten wobei nicht in weniger als etwa 25 Minuten die Energiemenge erreicht ist, welche eine negative Krümmung verursacht gar keine negative Krümmung auftreten kann. Man kann also den Schlusz ziehen, dasz beischwachen Intensitäten, wie lange auch die Beleuchtung fortgesetzt wird, nie eine negative Krümmung erscheinen wird.

\section{§17. Die ,zweite positive Krümmung".}

Pringsheim ${ }^{1}$ ) und $\mathrm{Clark}^{2}$ ) haben einen Unterschied gemacht zwischen einer ersten und einer zweiten positiven Krümmung. Aus dem bereits Mitgeteilten geht hervor. dasz man bei denjenigen Intensitäten wo eine rein negative Krümmung auftritt wirklich eine dergleiche Scheidung machen kann.

Bei Intensitäten, welche schwächer als 100 M.K. sind wo aber doch noch eine negative Krümmung sichtbar wird, sind damit schon Schwierigkeiten verbunden da immer erst eine positive Krümmung auftritt. Wird hier eine negative Krümmung sichtbar, so ist sie schwach und tritt sie erst auf nachdem schon eine deutliche positive Krümmung erreicht worden ist. Bei den schwächsten Intensitäten ist es aber ganz unmöglich diese Scheidung zu machen, da hier nie eine negative Krümmung auftritt. Das Einführen der Namen erste und zweite positive Krümmung hat nur dann $Z_{\text {weck, }}$ wenn ein qualitativer Unterschied gezeigt

1) Pringsheim, E. Beitr. z. Biol. d. Pflanzen 9. 1907 1909. 10. 1913.

") Clark, O. L. Ueber negativen Phototropismus bei Avena sativa. Zeitschr. f. Bot. Bd. 5. 1913. 
werden kann. Es ist aber ganz unmöglich am Krümmungsverlaufe zu sehen, welche von den beiden sie ist. Die Angabe Clarks über die Dauer der Reaktionszeiten. welche für die erste positive Krümmung 1 Stunde für die negative 2 bis $2 \frac{1}{2}$ Stunde und für die zweite positive 1 Stunde $15 \mathrm{Min}$. betrüge, ist falsch, da entweder für die positiven Krümmungen oder für die negativen die Reaktionszeit konstant ist. Eine erste positive Krümmung kann ebensogut wie eine sogenannte zweite schon nach einer halben Stunde sichtbar werden, dieses wird aber wie schon besprochen ist von der Stärke der Reaktion abhängig sein. Eine rein negative Krümmung ist wie aus Fig. 9 hervorgeht schon nach 45 Minuten aufgetreten. Ebenso wenig ist in der Stärke der Krümmung ein Unterschied zu finden, da sowohl erste als zweite positive Krümmung in allen Stärken vorkommen. Wohl kann eine zweite positive Krümmung eine viel gröszere Stärke erreichen als je eine erste positive, wie z. B. die Krümmung zeigt welche in Fig. 4 auf Tafel I abgebildet worden ist; diese ist nach einer Beleuchtung von 45 Minuten mit einer Intensität von 5.5 M.K. entstanden. Doch auch das ist kein qualitativer Unterschied. Eben die Erscheinung, dasz bei sehr schwachen Intensitäten gar keine negative Krümmung auftritt und hier also erste und zweite positive Krümmung allmählig in einander übergehen, macht es wahrscheinlich dasz auch bei den stärkeren Intensitäten kein qualitativer Unterschied besteht.

Die mitgeteilten Beobachtungen geben viel mehr den Eindruck, dasz die negativen Erscheinungen sozusagen auf die positive Kr ümmungsuperponiertwerdenund dasznur dann, wenn die negative Reaktion sehrstark ist, die positive Krümmung supprimiert wird. Man hat sich also nicht mit Clark die Frage zu stelien: unter welchen Umständen tritt die zwcite positive Krüm- 
mung auf, sondern diese: warum kommt bei einen gewissen Reizungsdauer die negative Krümmung nicht zu Stande?

Aus den Zahlen von Tabelle 12 geht hervor, dasz das Sichtbarwerden der negative Reaktion bei einer bestimmten Energiemenge stattfindet. Nach Analogie könnte man sich abfragen ob auch das nicht mehr Auftreten der negativen Krümmung bei längerer Beleuchtung, von einer bestimmten Energiemenge abhängig sei.

In Tabelle 12 (p. 85) sind auch die Energiemengen, wobei die negative Krümmung nicht mehr auftritt, zusammengestellt worden. Unzweifelbar sind die Energiemengen nicht für alle Intensitäten dieselben. Es scheint ein Maximum bei der Intensität von 450 M.K. zu liegen da sowohl bei stärkeren als bei schwächeren Intensitäten eine kleinere Energiemenge das Ende der negativen Reaktion bestimmt. Vergleicht man die in der selben Tabelle aufgenommenen Werte $\mathrm{Clarks}$ für den Anfang der zweiten positiven Krümmung so ist eine Uebereinstimmung da, insoweit auch seine Ergebnisse darauf weisen, dasz diese Erscheinung nicht bei konstanter Energiemenge auftritt. Die Unterschiede sind aber wichtig genug um näher besprochen zu werden.

Ein Teil kann der Tatsache zugeschrieben werden, dasz $\mathrm{Clark}$ negative Krümmungen und geotropische Gegenkrümmungen nicht unterscheidet z. B. bei 1,25 M.K. Seine Werte für die Intensitäten 5 und 16 M.K. sind in genügender Ulebereinstimmung aber bei denjenigen von 100 M.K. und mehr sind die Differenzen grosz. Es ist nicht möglich für diese Abweichungen eine Erklärung zu finden. Nur kann darauf hingewiesen werden, dasz die Werte der zwei höchsten Intensitäten womit hier gearbeitet worden ist und welche nicht von Clark untersucht worden sind obwohl sie sehr ungenau sein können doch in dieselbe Richtung weisen. Auszerdem stimmen sie sehr 
gut mit den von Blaauw mitgeteilten Ergebnissen überein der auch bei diesen Lichtmengen positive Krümmungen erzielt hat.

Bla a u gibt in Tabelle 10 für eine Intensität von 400 M.K. an, dasz bei 182000 M.K.S. eine positive Krümmung auftritt. Dieser Wert stimmt besser mit dem hier gegebenen von 135000 M.K.S. als mit demjenigen Clarks 480000 M.K.S. ; ebenso fand Bla a u w für eine Intensität von 40000 M.K. bei 40000 M.K.S. eine schwache positive Krümmung, indem hier bei einer Intensität von 20000 M.K. bei 20000 M.K.S. eine positive Krümmung erhalten wurde.

Mit den Wahrnehmungen worauf sich die Zahlen für das Ende der negativen Krümmung stützen, kann ein Fehler verbunden sein welche verursacht wird indem die Pflanzen nicht bei konstanter Temperatur gezogen worden sind. Da aber die Vorbehandlung der Pflanzen bei diesen Versuchen immer dieselbe war, ist keine Veranlassung da, zu erwarten dasz die Vergleichungsmöglichkeit der Pflanzen untereinander dadurch beeinfluszt worden ist.

In Tabelle 12 ist auch die Zeit aufgenommen, worin die Energiemengen zugeführt worden sind welche das Ende der negativen Krümmung bestimmen. Diese Zeiten sind kleiner je nachdem die Intensität womit gereizt wurde gröszer ist. Der Prozesz, welcher das nicht mehr zu Stande kommen der negativen Krümmung verursacht, musz also abhängig sein von der Intensität womit gereizt wird.

$\S$ 18. Eine zweite Abnahme der Stärke der positiven Reaktion bei starken Intensitäten.

Aus den Tabellen 10 und 11 geht hervor, dasz bei diesen starken Intensitäten eine zweite Abnahme der positiven Reaktion gefunden wird. In Tabelle 10 ist für die Intensität von ungefähr 4600 M.K. angegeben, dasz die 
Krümmung nach einer Beleuchtung von 15 Minuten (4140000 M.K.S.) schwächer ist als bei Beleuchtungen von 16 und 30 Sekunden. In Tabelle 11 für eine Intensität von ungefähr 20000 M.K. wird bei einer Reizdauer von 4 Minuten keine Reaktion sichtbar, während bei kürzerer Reizdauer (2 und 10 Sek.) eine positive Krümmung erscheint. Auch in der hier aufgenommenen Tabelle Blaauws für 40000 M.K. tritt nach einer Minute keine Reaktion auf, wohl nach einer Sekunde. Es ist also bei Lichtmengen welche noch gröszer sind als diejenigen wobei positive Krümmung auftritt wieder eine Abnahme der Reaktionsstärke zu beobachten. Bei Clark und Pringsheim sind über diese Erscheinung keine Data zu finden.

\section{§19. Einflusz der Temperatur auf die negative Krümmung.}

Ueber den Einflusz der Temperatur auf die phototropische Empfindlichkeit sind einige Tatsachen durch die Untersuchungen von $\mathrm{Nijbergh}{ }^{1}$ ) und Fräul. $\mathrm{M}$. de Vries ${ }^{2}$ ) bekannt geworden. Beide haben nur ihren Einflusz auf die positive Reaktion studiert und sind zu ganz abweichenden Resultaten gekommen, Nijbergh fand dieselbe Empfindlichkeit für alle Temperaturen, Fräul. de Vries eine starke Abhängigkeit von der Temperatur wobei die Regel van 't Hoffs gelten würde. In Uebereinstimmung mit dieser letzten Untersuchung ist berücksichtigt worden, dasz die Temperatur einen sehr groszen Einflusz auf die Perzeption haben kann; deshalb haben die Versuche soviel wie möglich bei konstanter Temperatur statt gefunden.

1) Nijbergh, T. Über die Einwirkung der Temperatur auf die tropistische Reizbarkeit etiolierter Avena Keimlinge. Ber. D. Bot. Ges. Bd. 30. 1912.

) de Vries, M. Proc. Kon. Ak. v. Wet. Amsterdam. 1913. 
Auch im Laufe dieser Untersuchungen hat es sich gezeigt, dasz es sehr erwünscht ist grosze Sorge auf die Temperatur zu verwenden. Einige Data weisen darauf, dasz es wünschenswert wäre die Pflanzen bei konstanter Temperatur wachsen $z u$ lassen und bei derselben Temperatur die Versuche geschehen zu lassen, da die Empfindlichkeit und die Wachstumsgeschwindigkeit sich relativ langsam einstellen. Aus diesem Grunde haben eine grosze Anzahl Versuche bei derselben Temperatur statt gefunden, wobei die Pflanzen gewachsen waren. Es wird sehr erwünscht sein, dasz auch der Einflusz der Temperatur auf die negative Krümmung untersucht wird. 'Der Anfang der negativen Krümmung ist ein äuszerst empfindliches Reagenz für den Einflusz äuszerer Umstände auf den Reizprozesz. Vielleicht wird es möglich sein etwas mehr über die Beziehung von positiver $z u$ negativer Reaktion $z u$ erkennen indem man die Aenderung der Kảrdinalpunkte bei verschiedenen Temperaturen untersucht. 


\section{ABSCHNITT III. \\ Die Empfindlichkeit der Basis.}

\section{$\S 20$. Methode.}

Darwin ${ }^{1}$ ) hat schon darauf hingewiesen, dasz ein groszer Unterschied besteht zwischen der Spitze und der Basis von Avena Keimlingen. Selbst meinte er dasz die Basis gar nicht lichtempfindlich sei. Rothert ${ }^{2}$ ) hat in zahlreichen Versuchen gezeigt, dasz auch die Basis eine gewisse Empfindlichkeit besitzt obwohl sie viel weniger empfindlich sein würde als die Spitze. Van der $W o l k^{3}$ ) und Wilschke $e^{4}$ haben versucht die kleinste Lichtmenge $z u$ bestimmen, welche nötig ist um, wenn nur die Basis beleuchtet wird eine Krümmung $z u$ veranlassen. Beide haben gefunden, dasz eine Menge von 20000 M.K.S. erfordert wird um eine Krümmung der Basis zu bekommen. $\mathrm{Da}$ eine Menge von 10-20 M.K.S. eine Spitzenkrümmung verursacht schlieszen sie $z u$ einem groszen Unterschied in Lichtempfindlichkeit zwischen Spitze und Basis. Van der Wolk fand diesen Wert bei Beleuchtung der Hälfte des Keimlings, W ilschke beleuchtete Zonen von

1) Darwin, Ch. and F. The power of movements in plants. 1880.

2) Rothert, W. Ueber Heliotropismus. Beitr. z. Biol. der Pfl. Bd. VII. 1894.

i) Van der Wolk, P. C. Proc. Kon. Ak. v. Wet, Amsterdam. 1911.

4) Wilschke, A. Ueber die Verteilung der phototropischen Sensibilität in Gramineenkeimlingen und deren Empfindlichkeit für Kontaktreize. Sitz. ber. Kais. Ak. Wien. Math. naturw. Klasse. Bd. CXXII. Abt. I. 1913. 
2. $\mathrm{mM}$. und fand über die ganze Basis dieselbe Empfindlichkeit. Auch v. Guttenberg ${ }^{1}$ ) hat bei Versuchen über akropetale Reizleitung gefunden, dasz eine Beleuchtung von 40000 M.K.S. in einer Stunde zugeführt eine deutliche Krümmung der Basis verursacht.

Wilschke hat auf die von Rothert, van der Wolk und v. Guttenberg benutzte Methode kritik geübt. Er meint, dasz nur mit seiner Methodik zuverlässige Resultaten $z \mathfrak{u}$ bekommen seien. Bei seiner Aufstellung fiel das Licht durch eine Spalte auf denjenigen Teil der Pflanzen dessen Lichtempfindlichkeit $z u$ bestimmen war. An dieser Methode haften einige Beschwerden. Nur wenige Pflanzen können auf diese Weise zugleicherzeit beleuchtet werden. Um auszerdem eine genaue Beleuchtung einer bestimmten Zone $z \mathfrak{u}$ bekommen musz die Entfernung von der Pflanze zur Spalte äuszerst klein sein und dadurch entsteht eine Gefahr für Kontaktkrümmungen. $\mathrm{Ob}$ bei dieser Methode, wenn eine breitere Spalte als bei den Versuchen Wilschkes benutzt wird keine Gefahr entsteht dasz die Spitze schwach beleuchtet wird, scheint keineswegs ausgeschlossen.

$\mathrm{Da}$ bei den Versuchen welche hier über diese Frage mitgeteilt werden mit derselben Methode gearbeitet worden ist als bei denjenigen Rotherts, van der Wolks und $\mathrm{Guttenbergs}$ ist es erwünscht die Beschwerden welche Wilschke anführt näher zu betrachten. Seine Beschwerde beruht hauptsächlich auf der Empfindlichkeit der Avena Keimlinge für Kontaktreize welche insbesondere von van der Wolk besprochen worden ist. Wilschke hat diese traumatische Erscheinung näher analysiert. Er fand dasz die oberen $4 \mathrm{mM}$. eines Keimlings gar nicht empfindlich für Kontaktreize waren und dasz bei niedrigeren

1) Guttenberg, H. von. Ueber akropetale heliotropische Reizleitung. Jahrb. f. Wiss Bot. Bd. 52. 1913. 
Zonen 10 mal kräftig mit einem rauhen Hölzchen gerieben werden muszte um eine Krümmung zu bekommen. Eine Reizleitung hat er nie beobachtet.

Die von Wilschke kritisierte Methode ist das Stellen von Käppchen von Stanniol oder Alumin über die Spitze der Keimlinge. Man könnte aber gerade auf Grund seiner Versuche gegen die Benutzung von Käppchen von 4-5 $\mathrm{mM}$. wie v. Guttenberg sie verwendete gar keine Beschwerden anführen. Noch viel weniger, wenn es nicht ein Druck sondern die Reibung ist welche die traumatische Krümmung verursacht. Bei längeren Käppchen wird es sicher unerläszlich sein grosze Vorsicht anzuwenden. Doch auch hier wird diese Methode, wenn nur mit genügender Vorsicht angewendet, keine unüberwindlichen Schwierigkeiten verursachen. Vorläufig ist sie nicht zu entbehren, da sie es möglich macht mit vielen Pflanzen zu gleicher Zeit zu arbeiten. Wenn man die geringe Laesion durch das vorsichtige Aufsetzen des Käppchens verursacht, vergleicht mit den von Rothert ${ }^{3}$ ) Fitting ${ }^{2}$ ) und Jost ${ }^{3}$ ) bei ihren Versuchen bewirkten Verwundungen, dann scheint es keineswegs so unerlaubt aus den mit der Käppchenmethode bekommenen Beobachtungen Folgerungen zu ziehen. Es hat sich auszerdem noch gezeigt, dasz es mit der Methode Wilschkes nicht möglich ist die Ergebnisse zu bekommen welche hier beobachtet sind, da es gar nicht dasselbe ist ob man nur eine kleine Zone oder einen viel gröszeren Teil beleuchtet.

Die für diese Versuche benutzten Käppchen sind 5 oder $12 \mathrm{mM}$. lang und von Stanniol verfertigt. Ein Ende ist zugekniffen und für einen guten Lichtabschlusz

1) Rothert 1. c.

2) Fitting, H. Jahrb. f. Wiss. Bot. Bd. 44. 1907. Die Leitung tropistischer Reize u. s. w.

3) Iost. Zeitschr. f. Bot. Bd. 4. 1912. Studien über Geotropismus. 
mit Lacke umgeben. Die dadurch entstehene Verdickung erleichtert das Aufsetzen der Käppchen, so dasz sie fast ohne Reibung um die Spitze gestellt werden können, da sie einen etwas gröszeren Diameter haben als der Keimling; sie ruhen auf der Spitze.

Bei diesen Versuchen musz auszerordentlich viel Sorgfalt auf das Material verwendet werden, denn um zuverlässige Ergebnisse $z \mathrm{u}$ bekommen ist es absolut notwendig nur Pflanzen zu benutzen welche vollkommen gerade gewachsen sind. Das verursachte, dasz fast immer 80 bis $90 \%$ des gezogenen Materials für untauglich erklärt werden muszte.

\section{$\S 21$. Positive und negative in der Basis induzierte Krümmungen.}

Die gröszte Anzahl der Versuche ist mit Käppchen von 4 und $5 \mathrm{mM}$. ausgeführt worden. Ueber diese ist hier zuerst zu berichten. Tabelle 14 gibt eine Uebersicht über die Ergebnisse dieser Versuche. Diese Beobachtungen sind schwierig da insbesondere nach kurzdauernden Beleuchtungen nur schwache und undeutliche Krümmungen erscheinen. Dadurch war es nicht möglich darauf $z u$ achten in welchen Zonen die Krümmung sich befindet. Wohl bekommt man den Eindruck, dasz bei schwachen Krümmungen die höheren Zonen einen geringen Anteil an der Krümmung haben. Bei starken Krümmungen sind aber alle beleuchteten Zonen deutlich gekrümmt.

Die Ergebnisse von Tabelle 14 müssen mit denjenigen von $\S 14$ wo die Spitzenbeleuchtungen besprochen worden sind verglichen werden. Es zeigt sich, dasz e ine grosze Uebereinstimmung $z$ wischen dem Effekt von Beleuchtungen der Spitze und der Basis be$\mathrm{s}$ te $\mathrm{h}$ t. Es ist besonders auffallend dasz im Widerspruch mit den Mitteilungen van der Wolks und Wilschkes die Empfindlichkeit der Basis ziemlich grosz ist. Die 


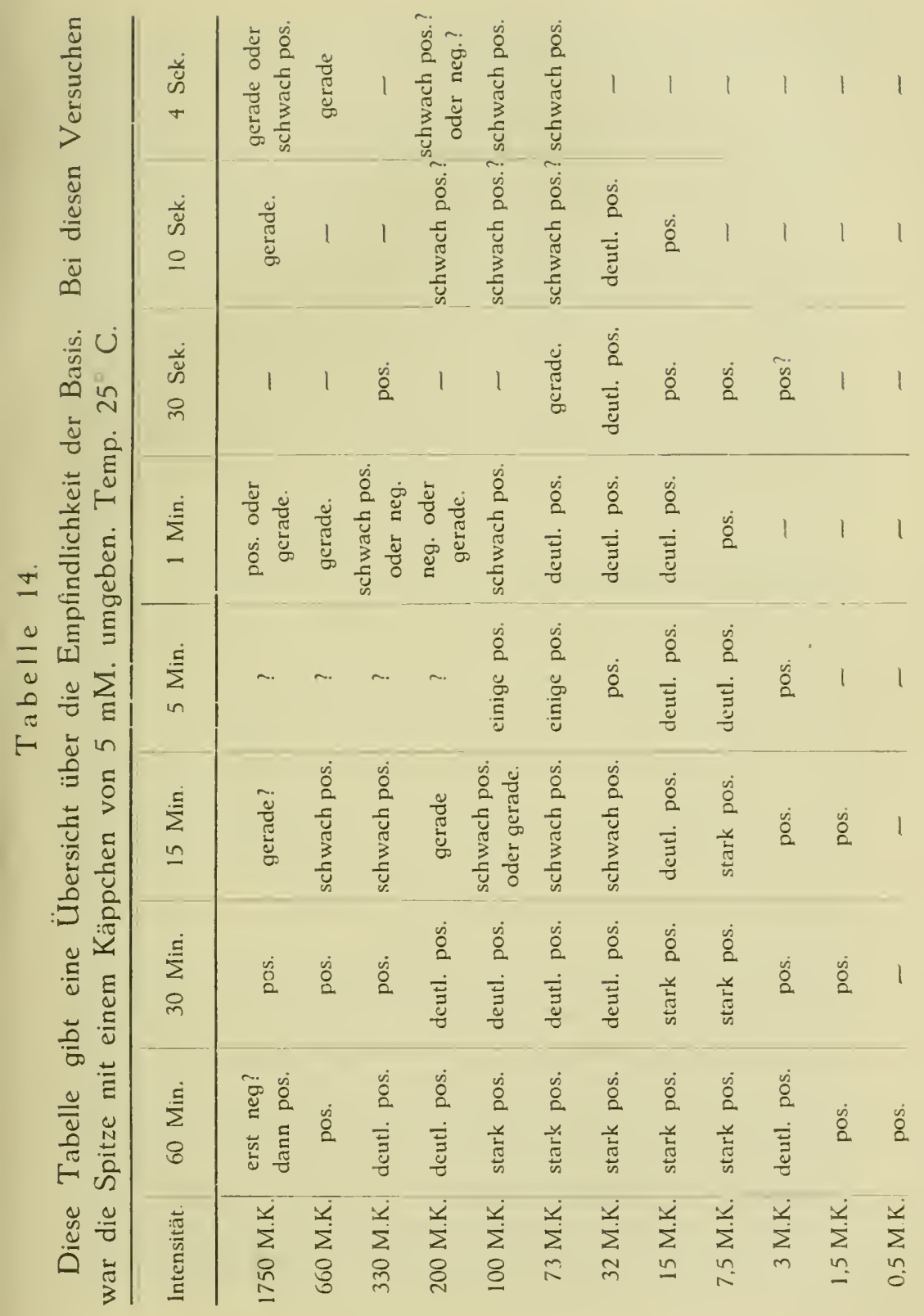


eben sichtbare Krümmung befindet sichnicht bei 20000 M.K.S. sondern bei etwa 100 M.K.S. (vergl. Tafel I Fig. 5.) Damit verschwindet also groszenteils der Unterschied in Empfindlichkeit, welchen frühere Untersucher so stark in den Vordergrund gestellt haben.

Ebenso wie bei Spitzenbeleuchtungen sieht man auch hier bei schwachen Intensitäten bei jeder Reizdauer eine positive Krümmung auftreten. Bei den stärkeren tritt, ähnlich wie das früher ausführlich auseinandergesetzt worden ist bei einer gewissen Reizdauer Abnahme der Stärke der positiven Krümmung auf. $\mathrm{Ob}$ auch negative basale Krümmungen auftreten können ist nicht mit Sicherkeit festgestellt worden. Wohl sind dergleichen Krümmun gen regelmäszig bei bestimmten Lichtmengen beobachtet worden aber die Möglichkeit besteht dasz hierbei auch Kontaktkrümmungen und Nutationen aufgetreten sind. Nutationen haben diese Untersuchung sehr erschwert; bei der relativ hohen Temperatur waren sie hinderlicher als je. (25 à $26^{\circ}$ Cels.) Dennoch schien es erwünscht bei dieser Temperatur die Versuche anzustellen; denn wenn die Krümmungsstärke eine Funktion der Wachstumsgeschwindigkeit ist kann man den besten Erfolg erwarten, wenn die Temperatur so hoch ist, dasz ein schnelleres Wachstum auftritt; so werden die Einzelheiten des Krümmungsprozesses wie vergröszert.

Eine starke Intensität gibt also wie bei den Spitzenkrümmungen bei sehr kurzer Reizdauer eine positive Krümmung, bei etwas längerer kommt unter dem Einflusz der negativen Reaktion eine Abnahme der positiven zustande, sodasz keine (oder negative?) Krümmung auftritt. Bei noch länger fortgesetzten Beleuchtungen wird wieder eine positive Krümmung sichtbar (sogenannte 2. positive Krümmung). Alle Krümmungen welche bei langer Reizdauer erscheinen, sind da bei langer Reizdauer die negative Einflusz geringer ist, viel stärker als diejenigen bei 
kürzeren Reizungen auch wenn mit derselben Energiemenge beleuchtet worden ist. Der Unterschied mit der Spitzenreaktion liegt hauptsächlich darin. dasz bei der Basis die sogenannte 1. positive $\mathrm{Krümmung} \mathrm{so} \mathrm{schwach} \mathrm{ist.} \mathrm{Da} \mathrm{es} \mathrm{sehr} \mathrm{schwierig}$ ist diese erste positive Krümmung zu erzielen (die richtige Energiemenge und gutes Material) ist eine derartige Krümmung photographiert und hier in Tafel I Fig. 5 aufgenommen worden. Bei allen ist die Basis schwach aber deutlich nach links gekrümmt. (bei den am stärksten basal gekrümmten zeigt die Spitze eine schwache entgegengesetzt gerichtete geotropische Aufrichtung.) Bei Beleuchtung während längerer Zeit z. B. einer Stunde ist es möglich bei einer Intensität von $0.5 \mathrm{M}$.K. noch eine deutliche positive Krümmung zu bekommen.

Mit den in Tabelle 14 gegebenen Werten sind die Ergebnisse van der Wolks und v. Guttenbergs keineswegs im Streit. Van der Wolk sah bei etwa 20000 M.K.S. basale Krümmungen. Aus der hier gegebenen Tabelle folgt dasz er dann länger als 5 Minuten gereizt hat. Wie man auch das Produkt von 20000 M.K.S. bekommt, wenn nur die Reizdauer gröszer ist als 5 Minuten, stets entsteht eine deutliche positive Krümmung. Wird 20000 M.K.S. in weniger als 5 Minuten zugeführt, so resultiert entweder gar keine Krümmung oder eine negative. V. Guttenberg bekam bei 40000 M.K.S. in einer Stunde zugeführt, eine starke positive Reaktion; das stimmt auch genau mit den schon mitgeteilten Werten überein. Ueber die Versuche Wilschkes ist wenig mit Sicherheit zu sagen. Es ist sehr wahrscheinlich, dasz bei Beleuchtung einer so kleinen Zone von $2 \mathrm{mM}$. die Krümmungen welche doch schon so schwach sind nicht makroskopisch sichtbar werden. Es ist sicher empfehlenswert diese Krümmungen mit Hilfe eines Mikroskopes eventuell auf einem Klinostaten auszuspüren. 
Ohne die Ergebnisse, welche $\mathrm{W}$ ilschke erreicht hat auch nur im geringsten in Zweifel zu ziehen, musz man doch sagen dasz es vollkomen ungestattet ist mit ihm die Folgerung zu ziehen, dasz alle Zonen dieselbe Empfindlichkeit haben, da 20000 M.K.S. eine Krümmung bei allen verursacht. Dergleichen Folgerungen müssen sich auf eine gedehntere Kenntnis vom Reaktionsvermögen stützen insbesondere bei kurzdauernden Reizungen. Dies geht ganz deutlich aus einigen Versuchen hervor, welche zum Vergleich mit der Methode Wilschkes angestellt worden sind und wobei einer Zone von 2 mM. 20000 M.K.S. in kurzer Zeit zugeführt wurde: die Basis krümmte sich nicht. Man kann also nicht 20000 M.K.S. als Empfindlichkeitsgrenze für die Basis angeben.

Auch für diese basalen Krümmungen gelten die Betrachtungen welche bei Spitzenbeleuchtungen die Unmöglichkeit gezeigt haben den Schwellenwert und die theoretische Reaktionszeit zu bestimmen. Die experimentelle Reaktionszeit ist auch hier vonder Stärke der Krümmung abhängig. Bei Durchbeleuchtung mit 15-32 M.K. ist nach 30 Minuten die Krümmung sichtbar.

Eine schwache Krümmung erscheint aber erst nach $11 / 2$ Stunde. Im Vorbeigehen musz noch darauf hingewiesen werden, dasz die Angabe Wilschkes „Bemerkenswert ist, dasz die Präsentationszeit, ähnlich wie sie Vouk für den negativen Phototropismus bei Wurzeln fand, fast so lang ist wie die Reaktionszeit, wenn man als Reaktionszeit die Zeit vom Ende der Exposition bis zum Eintritte einer merkbaren Krümmung betrachtet" durch die hier mitgeteilten Beobachtungen allen Grund verliert.

Die Versuche mit Käppchen von $12 \mathrm{mM}$. bieten nach dem oben Mitgeteilten wenig Neues. Bei 400 M.K.S. in 4 Sekunden zugeführt tritt eine schwache positive Krümmung auf, bei $1000-2400$ M.K.S. (in weniger als 1 
Minute) besteht eine Neigung $z u$ negativen Krümmungen aber auch hierauf kann nicht viel Wert gelegt werden, da die negative Krümmungen so schwach bleiben und dadurch die Kontaktkrümmungen nicht ausgeschlossen werden können.

Wenn man die Versuche dieses Abschnittes zusammenfaszt, kann man sagen dasz die Erscheinungen bei Beleuchtung der Spitze und Basis eine sehr grosze Uebereinstimmung zeigen, dasz aber die basalen Krümmungen viel weniger deutlich sind. Der grosze Unterschied den man immer zwischen Spitze und Basis gefunden hat, ist dadurch verursacht worden dasz man die 1. positive Krümmung und das Gebiet der negativen Reaktion nicht bemerkt hat. Man bestimmite also die Schwelle für die zweite positive Krümmung. Es ist unmöglich in Zahlen den Unterschied in Empfindlichkeit zwischen Spitze und Basis auszudrücken, da dann ungleichartige Krümmungen verglichen werden müssen.

Es ist ebenso wenig möglich Schwellenwerte zu vergleichen, da es selbst nicht annähernd festzustellen ist, wieweit die kleinste sichtbare Reaktion von dem Schwellenwert entfernt ist. Die Erscheinungen machen es wahrscheinlich, dasz das Perzeptionsvermögen der Basis nicht so ganz abweichend ist von dem der Spitze. Das wird später noch näher besprochen werden. (\$ 46).

§22. Ueber den Einflusz von Beleuchtungen von Spitze und Basis a uf einander,

$\mathrm{Van}$ der Wolk hat den Einflusz untersucht, welchen eine Beleuchtung der Basis auf die Empfindlichkeit der Spitze und eine der Spitze auf diejenige der Basis hat. In beiden Fällen meinte er zeigen zu können, dasz die Empfindlichkeit vergröszert werde. Diese Mitteilung enthält aber keine Data über die Weise, worauf er diese Erscheinung festgestellt hat. 
Es ist also in Ermangelung genügender Tatsachen nicht möglich seine Versuche zu wiederholen. v. Guttenberg hat es versucht; er fand aber dasz eine vorhergehende Beleuchtung der Basis die Spitze für eine Beleuchtung der entgegengesetzten Seite nicht empfindlicher macht, sondern genau den umgekehrten Effekt zu Folge hat. Er schlieszt aus seinen Versuchen nicht zu einer verminderter Empfindlichkeit der Spitze, sondern zu einer akropetalen Reizleitung, wobei der von der Basis zur Spitze geleitete Reiz die entgegengesetzt gerichtete Spitzenkrümmung vermindert. Die im vorigen Abschnitte mitgeteilten Versuche über die Empfindlichkeit der Basis haben Veranlassung gegeben diese Experimente noch ein $\mathrm{Mal}$ zu wiederholen da es möglich schien eine Erklärung für die von $\mathrm{v}$ an der Wolk erzielten Ergebnisse zu finden.

Es ist sicher, dasz Van der Wolk mit groszen Energiemengen gereizt hat.

Nun ist es wahrscheinlich gemacht, dasz diese bei kurzer Reizdauer eine negative basale Krümmung verursachen. Es könnte möglich sein, dasz diese negative basale Krümmung sich mit einer Spitzenkrümmung der anderen Seite zu einer stärkeren Krümmung von Spitze und Basis in der Richtung der Spitzenbeleuchtung summieren könnte. Mit dem Zwecke dieses zu untersuchen ist eine Reihe Versuche angestellt worden. Der Erfolg hat aber nicht befriedigt. Eine starke Krümmung resultierte nie. Wohl ist in vielen Fällen eine kleine Vergröszerung der Spitzenkrümmung beobachtet worden, welche auch etwas früher sichtbar wurde, aber so eklatant wie va n d e r W o lk seine Resultate beschreibt war es nicht. Die Weise, worauf $\mathrm{van}$ der Wolk die Verstärkung der Krümmung gemessen hat, kann nicht glücklich gewählt genannt werden. Er spricht von einer maximalen Krümmung, welche in einer halben Stunde erreicht wird. Obwohl die Temperatur wobei er experimentiert hat sehr hoch ist $\left(27\right.$ a $28^{\circ}$ Cels.). 
so ist es doch unwahrscheinlich, dasz bei Beobachtung der Krümmungsstärke nach einer halben Stunde ein zuverlässiger Resultat zu erwarten wäre. Eben bei dergleichen kurzen Krümmungszeiten, ist der Unterschied in Krüm mungsstärke am geringsten und sind die Beobachtungen der gröszten Gefahr ausgesetzt ungenau zu werden.

Auch die Erscheinung, welche von Guttenberg beschrieben hat, dasz die Spitzenkrümmung nach Vor-

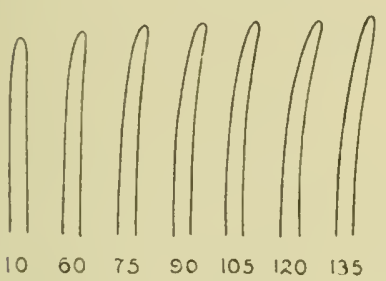

Fig. 11 .

Positiv phototropische Krümmung. Reizstärke 7,2 M. K.S. Temp. $25 \mathrm{C}$.

Die ganze Pflanze ist von rechts beleuchtet. Die Zahlen geben die Anzahl Minuten nach dem Anfang der Beleuchtung. Die Krümmung fand statt auf dem van Harreveldschen Klinostaten.

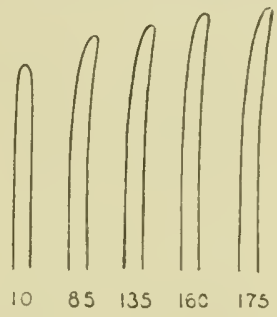

Fig. 12.

Positiv phototropische Krümmung. Reizstärke 7,2 M.K.S. Temp. $25^{3}$ C.

Nur eine Spitzenzone von $1 \mathrm{mM}$. beleuchtet.

Die Zahlen geben die Anzahl Minuten nach dem Anfang der Beleuchtung. Die Krümmung fand statt auf dem van Harreveldschen Klinostaten.

beleuchtung der Basis schwächer sei, ist näher geprüft worden. Es hat sich bei der Wiederholung dieser Versuche gezeigt, dasz es sehr schwierig ist Sicherheit zu bekommen ob die Spitzenkrümmung wirklich in der Spitzenzone geringer ist. v. Guttenberg führte der Basis 40000 M.K.S. in einer Stunde zu, und bekam wie schon oben mitgeteilt worden ist in guter Uebereinstimmung mit den Versuchen des vorigen Paragrafen deutliche positive Krümmungen der Basis. Wird nun auch 
die Spitze von der entgegengesetzten Seite beleuchtet, so wird man hier eine Gegenwirkung der nach der einen Seite gerichteten Spitzenkrümmung mit der entgegengesetzt gerichteten in der Basis induzierten Krümmung erwarten können. v. Guttenberg meint aber dasz auch
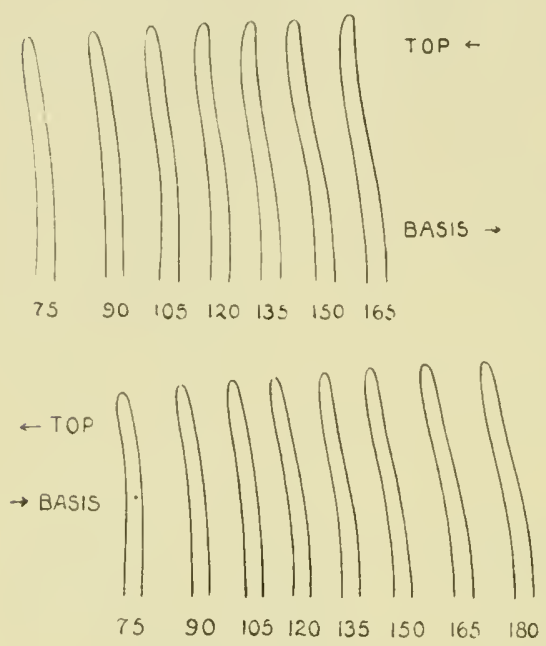

Fig. 13 und 14.

Während die Spitze mit einem Käppchen 15 mM.) verdunkelt ist wird die Basis während einer Stunde mit 11,8 M.K. beleuchtet. Am Ende dieser Beleuchtung wird das Käppchen hinweggenommen und die ganze Pflanze mit 7.2 M.K.S. von der entgegengesetzten Seite gereizt. Temp. $25^{\circ}$ C. Die Zahlen geben die Anzahl Minuten nach dem Anfang der ersten Beleuchtung. Nach dem Ende der Beleuchtung kommen die Pflanzen auf dem van Harreveldschen Klinostaten.

Reizleitung von den basalen Zonen nach der Spitze statt finde; also eine akropetale Reizleitung.

Eine Reihe Beobachtungen auf dem Klinostaten van $\mathrm{Har}$ revelds kann die mit der Lösung dieses Problemes verbundenen Schwierigkeiten näher verdeutlichen.

In Fig. 11 und 12 sind Krümmungen von Pflanzen abgebildet worden, welche ganz oder deren oberste Zone von $1 \mathrm{mM}$. mit 7,2 M.K.S. in 10 Sek. beleuchtet worden ist. $\left(25^{\circ}\right.$ Cels. $)$ Fig. 13 und 14 sind Krümmungen von Pflanzen, wobei zuerst die Basis von der einen Seite während einer Stunde mit 11,8 M.K. beleuchtet worden ist (42480 M.K.S.) und danach die ganze Pflanze von der entgegengesetzten Seite mit 7,2 M.K.S. Diese Abbildungen sind alle mit Hilfe des schon besprochenen 
Projektionszeichenapparates bei rotem Lichte verfertigt worden indem die Pflanzen sich auf dem intermittierenden Klinostaten befanden.

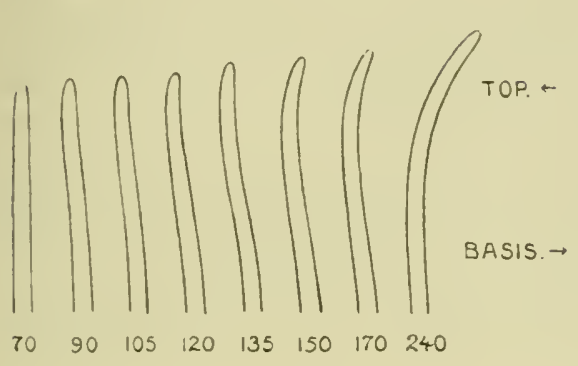

Fig. 15.

Während die Spitze mit einem Käppchen (5 mM.) verdunkelt ist, wird die Basis während einer Stunde mit 34 M.K. beleuchtet.

Am Ende dieser Beleuchtung wird das Käppchen hinweggenommen, und die ganze Pflanze mit 31 M.K.S. (in 15 Sek.) von der entgegengesetzten Seite gereizt. Temp. $25^{\circ} \mathrm{C}$.

Die Zahlen geben die Anzahl Minuten nach dem Anfang der ersten Beleuchtung.

Nach dem Ende der Beleuchtung kommen die Pflanzen auf dem van Harreveldschen Klinostaten.

Spitzenbeleuchtung so stark ist, dasz sie die entgegengesetzt gerichtete basale Krümmung, welche auch stärker ist als diejenige von Fig. 13 und 14 überwinden kann.

Man kann mit v. Guttenberg darin übereinstimmen, dasz die Empfindlichkeit der Spitze sich durch eine basale Vorbeleuchtung nicht geändert hat. Die Versuche geben aber keine Berechtigung $z$ u einer Reizleitung von Basis nach Spitze zu schlieszen. v. Guttenberg hat als Beweis Zeichnungen reproduziert welche 7 Stunden nach dem Anfange des Versuches verfertigt worden sind. Die hier mitgeteilten Beobachtungen zeigen, dasz schon nach zwei oder drei 
Stunden eine dergleiche schwache Spitzenkrümmung in Stärke abgenommen hat.

Van der Wolk hat auch mitgeteilt, dasz durch den Einflusz einer Spitzenbeleuchtung die Basis empfindlicher werde. Abgesehen von der Tatsache dasz nach Rothert Keimlinge ohne Spitze gleich nach der Verletzung unempfindlich für Lichtreizung sind, ist es nicht zu verwundern. dasz die Schwelle für basale Krümmungen unter 20000 M.K.S. gefunden wird. Wie oben gezeigt worden ist, befindet sich diese bei Pfianzen, welche noch eine Spitze besitzen, weit unter 20000 M.K.S. bei ungefähr 100 M.K.S. 


\section{ABSCHNITT IV.}

§23. Parallele unter verschiedenen Winkeln einfallende Strahlen.

Beim Anfang dieser Untersuchungen schien es erwünscht orientiert zu sein über die Wirkung von Lichtstrahlen, welche die Pflanze unter verschiedenen Winkeln treffen. Insbesondere bei längerdauernden Beleuchtungen, wobei während der Krümmung gereizt wird, ist es notwendig $z u$ wissen ob es als eine Quelle von Fehlern betrachtet werden musz, dasz die schräg stehende Spitze unter einem anderen Winkel getroffen wird. Die Versuche welche hierüber angestellt worden sind, werden nicht ausführlich besprochen werden, da sie jetzt nur eine Bestätigung einer vor kurzer Zeit erschienenen Mitteilung $\mathrm{Noacks}{ }^{1}$ ) sind. Seine Resultate weichen in Hinsicht auf die Stärke des Effektes bei kleinen Einfallswinkeln etwas ab, prinzipiell aber sagen sie auch, dasz die Wirkung von Strahlen welche unter verschiedenem Winkel mit dem Vertikal einfallen nicht nach der Sinusregel berechnet werden kann.

Diese Versuche sind mit einem parallelen Lichtbündel angestellt worden welches mittels einer Linse erzielt wurde. Als leuchtender Punkt diente ein durch einen Nernstbrenner beleuchtetes Milchglas, dessen Mitte nur, welche sich im Brennpunkte einer Linse befand, Licht ausstrahlen

j) Noack, K. Die Bedeutung der schiefen Lichtrichtung für die Helioperzeption parallelotroper Organe. Zeitschr f. Bot. Bd. 6. 1914. 
konnte. Der ganze Apparat war mit einem Köcher umgeben um das ungewünschte Licht abzuschlieszen und auf einem drehbaren Brette aufgestellt wodurch er in verschiedene Winkel gestellt werden konnte und zu gleicher Zeit die Richtung in der die Strahlen eine vertikal stehende Pflanze trafen, geändert wurde.

Um die Stärke des Effektes zu bestimmen ist nach der in $\S 8$ angegebenen Weise die Stärke der Krümmung, welche nach anderthalb Stunde erreicht wird, gemessen worden. Die Zahlen von Tabelle 15 sind jede das Mittel von etwa 20 Beobachtungen und geben für die verschiedenen Winkel, welche die Strahlen mit dem Vertikal machen die Krümmungsstärke bei verschiedener Reizdauer an.

Tabelle 15.

Unter verschiedenem Winkel einfallende Strahlen. Die Beleuchtungsstärke ist nicht bestimmt. In der Tabelle ist die Krümmungsstärke nach $1 \frac{1}{2}$ Stunde (in $\mathrm{mM}$. horizontale Spitzenabweichung) bei verschiedenem Einfallswinkel aufgenommen worden. Temp. $23^{\circ} \mathrm{C}$.

\begin{tabular}{|c|c|c|c|c|c|c|c|c|}
\hline \multirow{2}{*}{$\begin{array}{c}\text { Reizdauer } \\
\text { in } \\
\text { Sekunden. }\end{array}$} & \multicolumn{4}{|c|}{ Strahlen von oben. } & \multicolumn{4}{|c|}{ Strahlen von unten } \\
\hline & $15^{\circ}$ & $30^{\circ}$ & $50^{\circ}$ & $70^{\circ}$ & $90^{\circ}$ & $110^{\circ}$ & $130^{\circ}$ & $150^{\circ}$ \\
\hline 25 & 0.4 & 0.3 & 0.5 & 0,5 & 0.3 & 0.3 & 0 & - \\
\hline 35 & 0.7 & 0.7 & 0.9 & 0.8 & 0.6 & 0.4 & 0.2 & - \\
\hline 45 & 0.9 & 0.9 & 1 & 1.1 & 0.8 & 0.8 & 0.3 & - \\
\hline 55 & - & - & 1.5 & 1.6 & 1 & 0.9 & 0.4 & - \\
\hline 65 & - & - & - & - & 1.1 & 1.1 & 0.7 & - \\
\hline 85 & - & - & - & - & - & - & 1.1 & - \\
\hline 105 & - & - & - & - & - & - & 1.3 & - \\
\hline 200 & - & - & - & - & - & - & - & 0.5 \\
\hline 240 & - & - & - & - & - & - & - & 0.7 \\
\hline 300 & - & - & - & - & - & - & - & 1.1 \\
\hline 400 & - & - & - & - & - & - & - & 1.3 \\
\hline
\end{tabular}


Durch graphische Interpolation ist aus diesen Data die Zeit berechnet worden, während welcher in jeder Lage beleuchtet werden musz um nach $1 \frac{1}{2}$ Stunde eine Krümmung von $0,9 \mathrm{mM}$. zu bekommen.

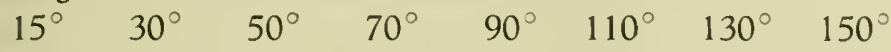
45Sek. 40Sek. 37 Sek. 37 Sek. 50Sek. 55Sek. 75Sek. 270Sek.

Aus diesen Zahlen darf man die Folgerung ziehen, dasz es einen groszen Unterschied macht ob die Strahlen von oben oder von unten kommen. Strahlen welche die Pflanze unter kleinem Winkel $d$, h. von oben treffen, wirken wenigstens ebenso stark wie horizontal einfallende Strahlen, während Strahlen welche unter einem Winkel gröszer als $90^{\circ}$ d. h. von unten einfallen eine ansehnlich schwächere Wirkung haben. No a ck hat ungefähr denselben Resultat bekommen. Er teilt aber mit dasz bei $9^{\circ}$ und $15^{\circ}$ eine gröszere Empfindlichkeit bestehe als bei $90^{\circ}$ und $45^{\circ}$. während hier bei $50-70^{\circ}$ ein Maximum gefunden worden ist.

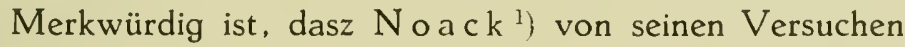
bei $9^{\circ}$ mitteilt ,die Krümmungen bei diesen Versuchen waren naturgemäsz nicht sehr stark". Er nimmt aber ganz aprioristisch an, dasz bei einem kleinen Winkel die Krümmungen weniger stark sein müssen obwohl doch eine kleine Reizmenge schon die Schwelle erreichen würde. Es zeigt sich dasz Noack nicht immer dieselbe Schwelle bestimmt hat und es möchte sehr gut möglich sein dasz dieses die Ursache seiner abweichenden Resultate ist. Es ist kein Versuch angestellt worden den von $\mathrm{Noack}$ erhaltenen Ergebnissen nachzuprüfen. Wenn wirklich wie er es sich denkt ein qualitativer Unterschied besteht in der Wirkung von Lichtstrahlen, welche einen verschiedenen Winkel mit dem Vertikal machen, wird es äuszerst schwierig sein zu entscheiden ob auch die Reaktion quali-

1) Noack l. c. p. 25 . 
tativ ${ }^{1}$ ) anders verlaufen wird und dadurch zustandebringen, dasz die Krümmung bei kleinen Einfallswinkeln nur eine geringe Stärke erreichen kann. Nach Noack würden seine Versuche beweisen, dasz die Richtung der Lichtstrahlen in der Pflanze Einflusz auf die Perzeption habe.

Eine sehr plausibele Erklärung scheint er aber nicht erwogen zu haben. Es ist bekannt, dasz eine Schwellenkrümmung ganz durch die Spitze des Keimlinges verursacht wird, da diese der empfindlichste Teil der Pflanze ist. Die Erklärung der obengenannten Erscheinungen kann nun in der Form der Spitze gefunden werden. Seinekonische Form macht dasz Strahlen, welche die Pflanze unter einem Winkel von $90^{\circ}$ treffen wohl dieganze Basis unter einem Winkel von $90^{\circ}$ beleuchten aber eben den empfindlichsten Teil der Pflanze unter einem Winkel, welcher gröszer ist als $90^{\circ}$. Die günstigste Lage wird diejenige sein, wobei die Strahlen einen möglichst groszen Teil der Spitze unter einem Winkel von $90^{\circ}$ oder fast $90^{\circ}$ treffen. Dieses Resultat ist bei $50-70^{\circ} \mathrm{zu}$ erwarten und wirklich stimmt dieses genau mit den Beobachtungen überein.

Bei dieser Betrachtung ist die Gültigkeit des Sinusgesetzes als selbstredend angenommen worden, da dieses nichts anderes aussagt als dasz die Energiemenge, welche die Pflanze pro Oberflächeneinheit trifft, vom Winkel worunter die Strahlen auf die Pflanze fallen abhängig ist. Das Sinusgesetz hat bei dem Phototropismus keine physiologische Bedeutung. Es ergibt sich logisch aus der Tatsache, dasz die Stärke der Perzeption durch die Ener-

1) Die Beleuchtungsumstände sind bei diesen kleinen Winkeln auch in so weit geändert, dasz je nachdem die Strahlen mehr senkrecht von oben kommen ein gröszerer Teil der Spitzenzellen welche an der entgegengesetzten Seite gelegen sind beleuchtet wird. 
giemenge bestimmt wird. Mit Noacks Folgerung, dasz Licht welches parallel mit der Längenachse die Pflanze trifft perzipiert wird, stimmt diese Betrachtung überein. Es wird dadurch verursacht, dasz die äuszerste Spitze unter einem Winkel von 90 gereizt wird und die konischen Seitenflächen unter einem kleineren Winkel. Eine Krümmung wird aber nicht entstehen können, da die Beleuchtung an allen Seiten gleich stark ist.

Bei Beleuchtungen von unten (Winkel gröszer als 90*) ist natürlich ein dauernd ungünstigerer Effekt zu erwarten da hier nicht nur die Energiemenge pro Oberflächeneinheit mit dem Sinus des Einfallswinkels abnimmt und durch Reflexion noch Licht verloren geht, sondern auch da die Spitze fast gar nicht mehr getroffen wird.

Es ist sehr bedauernswert, dasz diese einfache Erklärung von Noack nicht untersucht worden ist, da man dann gröszeren Wert auf seine Versuche mit Phycomyces legen könnte, wo man eine Gültigkeit des Sinusgesetzes eher erwarten würde, indem er auch hier Abweichungen gefunden hat. Diese Abweichung ist nur bei einem Winkel von ihm festgestellt worden, bei $150^{\circ}$ wo die Empfindlichkeit gröszer sein sollte als bei $90^{\circ}$. Diese einzige Abweichung ist aber nicht mit so groszer Sicherheit festgestellt worden dasz man zu einer Abweichung vom Sinusgesetze schlieszen müszte. Noack teilt mit, dasz er seine Versuche nicht bei konstanter Temperatur angestellt habe; aus der Untersuchung von Fräul. de Vries ${ }^{1}$ ) ist aber hervorgegangen dasz auch die phototropische Perzeptionsprozesse stark abhängig von der Temperatur sind. Bei Abwechslungen von verschiedenen Graden $\left(19-24^{\circ}\right)$ wie bei den Versuchen von $\mathrm{Noack}$ ist es unmöglich genau Schwellenbestimmungen auszuführen. Dabei kommt noch die schon früher besprochene grosze individuelle

1) de Vries, M. 1. c. 
Variabilität bei Phycomyces, welche es fast unmöglich macht sie zu quantitativen Versuchen zu benutzen. Der eine abweichende Wert kann also unter den Umständen, worunter er seine Versuche angestellt hat, nicht die Sicherkeit geben, dasz hier das Sinusgesetz nicht gültig sei. Eine erneute Untersuchung bei konstanter Temperatur wäre sehr erwünscht. 


\section{T E I L I I.}

\section{Mehrseitige Beleuchtungen.}

\section{$\S 24$. Einleitung.}

In diesem Teile werden Untersuchungen besprochen werden welche sich auf die Einwirkung verschiedener Reize auf einander beziehen. Insbesondere wird hier untersucht werden ob bei Zusammenwirkung mehrerer Reize die resultierende Reaktion zu einer Zusammenwirkung verschieden gerichteter einseitiger Reaktionen zurückgebracht werden kann und in welchem Masze neue Erscheinungen auftreten. Begreiflicherweise können die Resultate dieser Versuche sehr verwickelt sein.

Nicht nur bekommt man eine Vereinigung verschieden gerichteter positiver Reaktionen sondern auch von Kombinationen von positiven mit negativen Reaktionsprozessen. Es ist nicht gelungen so weit in die Analyse der Erscheinungen durchzudringen dasz es möglich geworden ist bestimmte Regeln aufzustellen nach welchen verschieden gerichtete einseitige Reize sich zu einer zusammengestellten Reaktion vereinen. Die hier folgenden Versuche werden denn auch nur mitgeteilt werden, weil sie mit genügender Sicherheit zeigen dasz man diese Erscheinungen auf einer $z u$ verwickelten Weise $z u$ untersuchen gepflegt hat, während es nötig und erwünscht ist dasz eine ganze Reihe einfacher Fragen erst zur Lösung gebracht werden. Erst wenn die Erscheinungen bei zweiseitigen entgegengesetzten Beleuchtungen quantitativ analysiert worden sind wird mit einem Studium der viel verwickelteren Rotationsversuche wie sie von $\mathrm{Pringsheim,} \mathrm{Clark} \mathrm{und} \mathrm{mir} \mathrm{ausgeführt}$ worden sind angefangen werden können. 


\section{ABSCHNITT V. \\ Zweiseitige Beleuchtungen.}

\$25. Gleichzeitige Beleuchtung von zwei entgegengesetzten Seiten.

$U_{m}$ die Zusammenwirkung verschieden gerichteter Reize zu untersuchen, ist es erwünscht mit der einfachsten Kombination anzufangen. Diese ist die Beleuchtung einer Pflanze von zwei entgegengesetzten Seiten. Das kann zu gleicher Zeit oder nach einander stattfinden. Zuerst wird die gleichzeitige Beleuchtung von zwei entgegengesetzten Seiten untersucht werden. Die Aufstellung für diese Versuche war sehr einfach. Im Dunkelzimmer im Gewächshause waren in einer Entfernung von zwei Meter zwei Metallfadenlampen von 10 Volt aufgestellt worden, welche auf einer Akkumulatorenbatterie bei konstanter Stromstärke brannten. Ihre Lichtstärke war $5 \mathrm{~K}$. Die geringen Dimensionen des Dunkelzimmers machten es unmöglich die Lampen in gröszerer Entfernung von einander zu stellen, wodurch die Genauigkeit der Wahrnehmungen gröszer gewesen wäre; auch war es durch Mangel an Akkumulatoren nicht möglich diese Versuche mit gröszerer Lichtstärke zu wiederholen. Die Versuche haben bei $23^{\circ}$ Cels. mit Material welches so viel wie möglich bei konstanter Temperatur gezogen war stattgefunden.

Die Pflanzen wurden in einer langen Reihe zwischen den beiden Lampen aufgestellt. Wird nun z. B. während 30 Sek. mit beiden Lampen zu gleicher Zeit beleuchtet so krümmen sich alle Pflanzen und genau in der Mitte 
zwischen den zwei Lichtquellen befindet sich der Punkt, von woher die eine Hälfte nach links, die andere nach rechts gekrümmt ist. Zuerst krümmen sich die Pflanzen, welche sich mehr der Lichtquelle nähern, aber nach etwa zwei Stunden ist keine ungekrümmte Pflanze mehr zu finden. Bei der starken Abnahme der Lichtintensität, welche bei dieser Aufstellung in der Mitte besteht, ist es schwierig zu bestimmen, wie weit eine Pflanze von der Mitte entfernt sein musz um sich zu krümmen. Mit Sicherheit ist konstatiert worden dasz diese Entfernung nicht mehr als 1 cM. beträgt $d$. h. dasz der Unterschied zwischen 152 und 147 M.K.S. noch zu einer Krümmung führt.

In Tabelle 16 sind die Ergebnisse dieser Versuchsreihe für verschiedene Dauer der gleichzeitigen Beleuchtung zusammengebracht worden, Um die Uebersicht zu erleichtern ist hier nur die Reaktion der einen Hälfte der Pflanzen mitgeteilt worden, welche also von der Mitte bis zu einer der Lichtquellen aufgestellt war. Eine positive Krümmung bedeutet, dasz eine Pflanze sich nach der Lichtquelle der sie sich am nächsten befand, hinkrümmte, eine negative. dasz sie sich von dieser hinwegkrümmte.

In Fig 16 sind die Resultate der Tabelle 16 schematisch abgebildet. Diese Figur hat nur den Zweck die Uebersicht zu erleichtern: Die Linien geben gar keine Idee der wirklichen Stärke der Krümmungen.

Tabelle 16.

Gleichzeitige Beleuchtung zweier entgegengesetzien Seiten.

Die Pflanzen befinden sich zwischen zwei Lichtquellen welche auf einer Entfernung von 2 Meter von einander aufgestellt worden sind, und welche eine Lichtstärke haben von $5 \mathrm{~K}$. Alle Pflanzen kamen nach der Beleuchtung auf den Klinostaten. $23^{\circ} \mathrm{C}$. 
Dauer der

Beleuchtung

\section{Sek. von der Mitte}

1 Min. von der Mitte bis zu 50 cM. 36 cM. \pm 2300 M.K.S.

2 Min. schwach positiv bis zu $50 \mathrm{cM}$. $50 \mathrm{cM}$. \pm 2400 M.K.S.

3 Min. schwach positiv bis zu $80 \mathrm{cM}$. $64 \mathrm{cM}$. \pm 2200 M.K.S.

4 Min. schwach positiv von $85 \mathrm{cM}$. bis zu $74 \mathrm{cM}$. deutlich von $80 \mathrm{cM}$. von der Lichtquelle 2350 M.K.S. 74 cM. \pm 2200 M.K.S.

5 Min. von 100 bis zu $90 \mathrm{cM}$. gerade $90 \mathrm{cM}$. \pm 1850 M.K.S. 10 Min. keine deutliche Krümmungen alle schwach negativ?

25 Min. von der Mitte positiv gekrümmt 60 Min. von der Mitte positiv gekrümmt
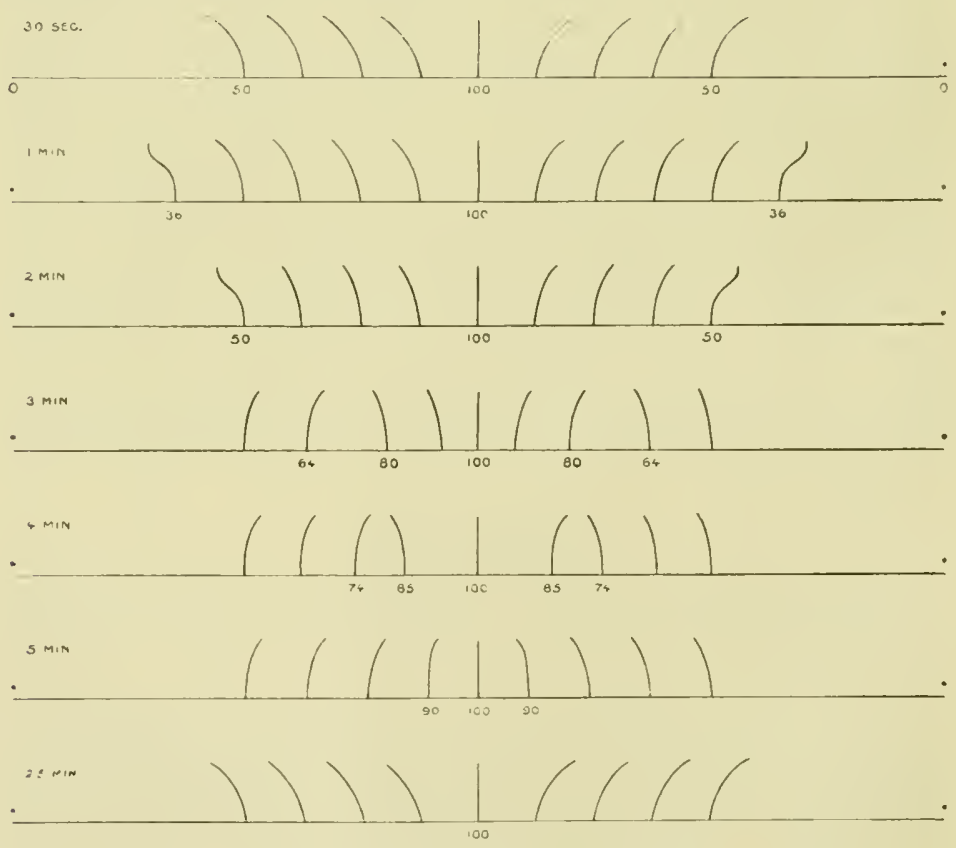

Fig. 16. Schematische Vorstellung der Tabelle 16. 
Die Beobachtungen welche in dieser Tabelle aufgenommen worden sind, sind einer selben Versuchsserie entnommen worden. Wenn man eine Vergleichung macht zwischen den Ergebnissen dieser Tabelle und denjenigen der Tabelle 13 ( $\$ 16$ p. 87) wo bei einer einseitigen Beleuchtung so viel wie möglich unter denselben Umständen gearbeitet worden ist '), dann scheint es erlaubt die Folgerung zu ziehen, dasz eine gleichzeitige $z$ weiseitige Beleuchtung sich wie eine Kombination von $z$ wei entgegengesetzt gerichteten einseitigen Beleuchtungen verhält.

Aehnlich wie bei einseitiger Beleuchtung bei schwachen Energiemengen eine positive, bei stärkeren (etwa 2000 M.K.S.) eine negative Krümmung auftritt, werden auch bei den gleichzeitigen zweiseitigen Beleuchtungen bei stärkeren Lichtmengen negative Krümmungen sichtbar.

Ueberlegt man sich z. B. was man erwarten kann bei einer gleichzeitigen Beleuchtung von zwei entgegengesetzten Seiten von 5 Minuten z. B. in einer Entfernung von $80 \mathrm{cM}$. von der linken Lichtquelle, so wird die linke Seite des Keimlings mit 2350 M.K.S., die rechte mit 1040 M.K.S. beleuchtet sein. 2350 M.K.S. würden als einseitige Beleuchtung eine starke positive Krümmung veranlassen, welche nach zwei Stunden in eine negative Asymmetrie übergeht; 1040 M.K.S. nur eine positive Krümmung. Als Resultat der kombinierten Krümmung tritt, wie aus der Tabelle 16 hervorgeht, eine deutliche negative Spitzenkrümmung hinsichtlich der linken Lichtquelle auf: d. h. die zwei positiven Krümmungen, welche nach links und rechts gerichtet sind äuszern sich nicht und nur die negative

1) Bei den Beobachtungen auf welche sich die Tabellen 4 bis 1 beziehen ist unter ganz anderen Umständen gearbeitet. Die Versuche dieses Abschnittes können nur mit den in $\$ 16$ des ersten Teils besprochenen einseitigen Beleuchtungen verglichen werden. 
Asymmetrie hinsichlich der linken Lichtquelle wird als negative Spitzenkrümmung sichtbar.

Aus den Versuchen mit einseitigen Beleuchtungen war hervorgegangen, dasz bei einer Beleuchtung von 25 Minuten und länger, nie etwas anderes als positive Krümmungen auftreten könnten (vergl. $\S 16$ ). Es ist damit in vollkommener Ulebereinstimmung, dasz bei einer gleichzeitigen Beleuchtung von zwei entgegengesetzten Seiten von 25 Minuten und länger (v. Tab. 16) nur positive Krümmungen auftreten; d. h. dasz die Pflanzen sich krümmen nach der Lichtquelle welche ihnen am nächsten ist. Auch in dieser Hinsicht schlieszt sich die zweiseitige Beleuchtung der einseitigen an.

Die Schwierigkeiten, welche sich bei der Analyse der Erscheinungen bei zweiseitiger Beleuchtung zeigen, beziehen sich insbesondere auf die Frage wie negative Krümmungen entstehen. Aus den hier in Tabelle 16 gegebenen Werten würde man geneigt sein abzuleiten, dasz die negative Reaktion bei ungefähr derselben Energiemenge (2000 2500 M.K.S.) sichtbar wird als bei einseitiger Beleuchtung (etwa 2000 M.K.S.) Es besteht aber eine Schwierigkeit insoweit es nicht gelungen ist mit Sicherheit festzustellen ob nicht auf andere Weise eine scheinbare negative Krümmung entstehen kann.

In Abschnitt I $\S 13$ ist mitgeteilt worden, dasz bei Reizen welche stärker sind als 300 bis 600 M.K.S. die maximale Krümmung schwächer wird. Die Frage ist nun ob wenn z. B. die linke Seite mit mehr als 600 M.K.S., die rechte Seite mit weniger beleuchtet wird, nicht eine Krümmung in der Richtung der kleinsten Energiemenge resultieren könnte, da die induzierte Krümmung an dieser Seite stärker ist. Bei einigen Versuchen, wobei aber die Vorgeschichte der Pflanzen leider unbekannt war, ist bei dergleichen Mengen eine Anweisung gefunden worden. dasz auf diese Weise eine scheinbar negative Krümmung 
entstehen kann. Da es aber bei Versuchen, welche mit diesem Zweck unternommen worden sind nicht gelungen ist diese Erscheinung mit Sicherheit festzustellen musz die Lösung dieses Problemes auf neue Untersuchungen warten. In Hinsicht auf die Erscheinungen, welche in den folgenden Paragrafen besprochen werden müssen, war es erwünscht hier auf diese Möglichkeit hinzuweisen.

Es scheint erlaubt aus den Beobachtungen, welche in diesem Paragrafen mitgesteilt worden sind, die Folgerung zuziehen, dasz bei einer zweiseitigen gleichzeitigen Beleuchtung das Zustandekommen einer Krümmung nicht durch den Unterschied der Energiemengen und ebenso wenig durch ihr Verhältnis bestimmt wird, da die negativen Krümmungen, welche hier auftreten, dann ganz unerklärbar sein würden. Dasz die zwei Reaktionen vollkommen in derselben Weise stattfinden, wie bei einseitigen Beleuchtungen, ist nicht wahrscheinlich da die Beleuchtungsumstände bei einer gleichzeitigen Reizung anders sein müssen als bei einseitigen Beleuchtungen. In der Hauptsache beruht aber die zweiseitige Beleuchtung auf einer Kombination zweier einseitiger.

\section{§26. Zwei Beleuchtungen von entgegen-} gesetzten Seiten nach einander.

Auch diese Versuche sind im Dunkelzimmer des Gewächshauses bei $23^{\circ}$ Cels. ausgeführt worden. Die zweiseitige Beleuchtung wurde auf zwei verschiedene Weisen erzielt. Zunächst indem eine Pflanze erst von einer Seite beleuchtet und danach $180^{\circ}$ gedreht wurde, so dasz die entgegengesetzte Seite ebenso stark beleuchtet wurde; an zweiter Stelle indem die Pflanzen, welche zwischen zwei Lichtquellen aufgestellt waren hintereinander mit diesen 
Lichtquellen gereizt wurden. Bei der ersten Methode (Tab. 17) wurde nur mit 2 bis 3 Pflanzen, welche sich in derselben Entfernung von der Lichtquelle befanden, zu gleicher Zeit gearbeitet, während bei den Versuchen der zweiten Tabelle (Tab. 18) eine Reihe Pflanzen zwischen den Lichtquellen aufgestellt war.

$$
\text { Tabelle } 17 .
$$

$Z$ wei Beleuchtungen von entgegengesetzten Seiten $n$ ach einander.

Intensität der Beleuchtung 5 M.K.

0 bedeutet nicht gekrümmt.

1 bedeutet gekrümmt nach der Lichtquelle der ersten Beleuchtung.

2 bedeutet gekrümmt nach der Lichtquelle der zweiten Beleuchtung.

1 bedeutet erst gekrümmt nach der Lichtquelle der ersten Beleuchtung und später nach derjenigen der zweiten.

\begin{tabular}{r|c|c|c|c}
\hline $\begin{array}{c}\text { Dauer der } \\
\text { ersten } \\
\text { Beleuchtung. }\end{array}$ & \multicolumn{4}{|c}{ Dauer der zweiten Beleuchtung. } \\
\cline { 2 - 5 } & 30 Sek. & 60 Sek. & 180 Sek. & 300 Sek. \\
\hline 30 Sek. & 0 & 2 & 2 & 2 \\
60 Sek. & 1 & 0 & 2 & 2 \\
& 1 & schwach 1 & 2 & 2 \\
180 Sek. & 2 & 2 & & \\
300 Sek. & 1 & 1 & 2 & 2 \\
\end{tabular}

In den Tabellen 17 und 18 sind die Resultate zusammengefaszt worden. Bei diesen zwei Tabellen betrug die Beleuchtungsstärke in einer Entfernung von $1 \mathrm{M}$. von der Lichtquelle 5 M.K. Bei allen Versuchen sind die 
Keimlinge sogleich nach der Beleuchtung auf den Klinostaten gestellt worden. Das hatte den Nachteil, dasz es experimentell schwieriger war das Auftreten einer Krümmung im Sinne der ersten Beleuchtung zu beobachten, so dasz es möglich ist dasz an einigen Stellen wo dieses nicht in den Tabellen aufgenommen worden ist doch äuszerst schwache Spitzenkrümmungen im Sinne der ersten Beleuchtung vorhergegangen sind. Wenn aber eine deutliche Krummung im Sinne der ersten Beleuchtung auftritt, ist das immer in den Tabellen erwähnt worden.

Aus der Tabelle 17 geht hervor, dasz nur wenn die zweite Beleuchtung schwächer ist als die erste, beide Reize in einer Reaktion sich äuszern können. In der Tabelle ist aufgenommen worden, dasz bei einer Beleuchtung von 60 Sekunden von rechts und danach 60 Sekunden von links gar keine Krümmung auftritt: in einigen Fällen ist aber beobachtet worden dasz nach ungefähr $\frac{1}{2}$ Stunde eine schwache Spitzenasymmetrie nach links und eine Stunde später eine nach rechts sichtbar wird, doch in den meisten Fällen sind diese $z u$ schwach um beobachtet $z u$ werden. Das Merkwürdigste in dieser Tabelle ist, dasz eine Beleuchtung von 180 Sekunden von der einen Seite der eine ebenso lange von der entgegengesetzten Seite folgt nur eine Krümmung in der Richtung der zweiten Beleuchtung verursacht. Diese selbe Erscheinung zeigt sich auch bei zwei Beleuchtungen von 300 Sekunden.

Tabelle 18 .

$Z$ wei entgegengesetzt gerichtete Beleuchtungen nach einander.

Bei beiden Beleuchtungen ist gleich lang gereizt worden.

Die Beleuchtungsstärke auf 1 Meter Entfernung von den zwei gleich starken Lichtquellen beträgt 5 M.K. Die Entfernungen beziehen sich auf der Lichtquelle der ersten Beleuchtung. Die Entfernung der zwei Lichtquellen betrug 2 Meter. Temp. $23^{\circ} \mathrm{C}$. 
Alle Pflanzen sind nach der Beleuchtung auf dem Klinostaten um horizontale Achse gedreht worden.

Dauer der Beleuchtung

Entfernung

Krümmung

30 Sek. nach $\frac{1}{2}$ Stunde $100-50$ schwach 1 Beleuchtung nach 2 Stunden 150-98 2 Beleuchtung

85-50 1 Beleuchtung

1 Min. nach $\frac{1}{2}$ Stunde $100-50$ schwach 1 Beleuchtung nach 2 Stunden $140-80 \quad 2$ Beleuchtung $80-50 \quad 1$ Beleuchtung

2 Min. nach $\frac{1}{2}$ Stunde $20-50$ sehr schwach 1 Beleuchtung nach 2 Stunden 170-148 Spitze schwach 1 Beleuchtung 148-50 2 Beleuchtung

3 Min. nach $\frac{1}{2}$ Stunde $85-50$ sehr schwach 1 Beleuchtung nach 2 Stunden $150-50$ 2 Beleuchtung

5 Min. nach 2 Stunden $150-50$ stark 2 Beleuchtung

10 Min. nach 2 Stunden 150-50 stark 2 Beleuchtung
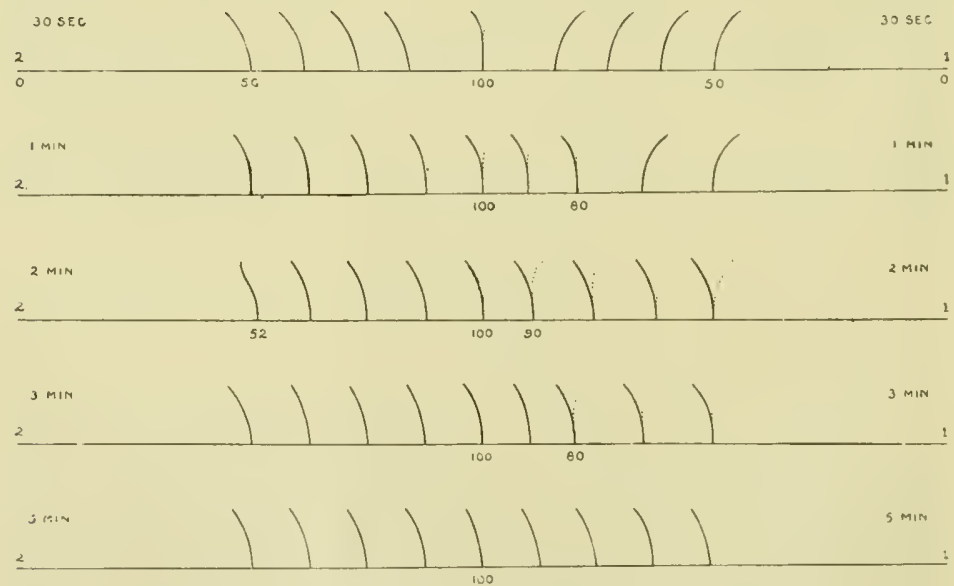

Fig. 17. Schematische Vorstellung der Tabelle 18. 
Bei den Versuchen der Tabelle 18 waren von $50 \mathrm{cM}$. von der linken Lichtquelle bis auf $50 \mathrm{cM}$. von der rechten, also über eine Strecke von 1 Meter eine Reihe Keimlinge aufgestellt worden. In der Fig. 17 sind die Resultate der Tabelle 18 schematisiert dargestellt worden. Diese Figur hat nur den Zweck die Uebersicht zu erleichtern. Die gezogenen Linien geben gar keine Idee in Bezug auf die wirkliche Stärke der Krümmungen. Die gestrichelten Linien zeigen dasz eine Pflanze sich erst in dieser Richtung krümmte und sich erst später in der durch die vollgezogene Linie angegebenen Richtung krümmte. In dieser schematischen Vorstellung ist angenommen worden dasz die erste Beleuchtung von rechts, die zweite von links stattfindet.

Das Ziel dieser Beobachtungen war zu untersuchen in welchem Masze auch nach vorhergehender Beleuchtung der einen Seite eine negative Krümmung hinsichtlich der zweiten Lichtquelle erscheint. Es ist bei dergleichen zweiseitigen Beleuchtungen selbstverständlich unmöglich, festzustellen ob eine Krümmung in der Richtung der Lichtquelle, womit die zweite Beleuchtung stattfindet, als eine negative hinsichtlich der ersten Beleuchtung betrachtet werden musz oder als eine positive hinsichtlich der zweiten Beleuchtung. Hingegen ist es wohl möglich $z \mathrm{u}$ beobachten ob wenn die zweite Beleuchtung mit Lichtmengen stattfindet welche als einseitige Beleuchtung eine positive Krümmung mit nachfolgender negativer Spitzenkrümmung verursachen würden auch noch nach vorhergehender Beleuchtung der anderen Seite diese negative Spitzenkrümmung erscheint. Bei einer Beleuchtung von 2 Minuten von der einen Seite und danach 2 Minuten von der anderen Seite tritt in einer Entfernung von $52 \mathrm{cM}$. von der Lichtquelle der zweiten Beleuchtung erst eine Krümmung auf, welche nach dieser Lichtquelle gerichtet ist und später eine Spitzenasymmetrie nach der Lichtquelle der ersten. Diese letzte wird hier als negative Krümmung hinsichtlich der zweiten 
Beleuchtung betrachtet werden müssen, da bei einer einseitigen Beleuchtung mit dieser Lichtmenge (2500 M.K.S.) gleichfalls zuerst eine positive und später eine negative Krümmung auftritt. Die vorhergehende Beleuchtung von 450 M.K.S. hatte keinen Einflusz auf das Zustandekommen einer negativen Krümmung hinsichtlich der zweiten Beleuchtung. Bei aufeinander folgenden Beleuchtungen von 3 Minuten war es aber nicht möglich negative Krümmungen hinsichtlich der zweiten Beleuchtung zu beobachten. Hier tritt immer nur eine starke Krümmung nach der Lichtquelle der zweiten Beleuchtung auf. Bei zwei aufeinander folgenden Beleuchtungen, welche jede 3 Minuten oder länger dauern zeigen sich also zwei merkwürdige Erscheinungen. Erstens bei Lichtmengen, welche jede für sich eine positive Krümmung geben würden aber über dem Maximum der positiven Reaktion sind, z. B. zwei Beleuchtungen von 900 M.K.S. tritt nur eine Krümmung in der Richtung der zweiten Beleuchtung auf (ungerechnet eine eventuell vorhergehende schwache Spitzenasymmetrie in der Richtung der ersten Beleuchtung.)

Zweitens, wenn die erste Beleuchtung mit einer Energiemenge stattfindet welche eine positive Krümmung geben würde, die zweite aber mit mehr als 2000 M.K.S. wobei also hinsichtlich der Lichtquelle der zweiten Beleuchtung eine positive Krümmung gefolgt von einer negativen Asymmetrie induziert würde, wird nur die Krümmung im Sinne der zweiten Beleuchtung sichtbar. Weder die positive Krümmung im Sinne der ersten Beleuchtung noch die negative hinsichtlich der zweiten zeigen sich.

Es kommt noch hinzu dasz wenn die erste und auch die zweite Beleuchtung stärker sind als 2000 M.K.S. und jede für sich also eine positive Krümmung, der eine negative Spitzenasymmetrie folgt, geben würde doch nur eine Krümmung gerichtet nach der Lichtquelle der zweiten Beleuchtung erscheint. 
Tabelle 19.

Aufeinander folgende Beleuchtung von zwei entgegengesetzten Seiten.

Intensität beider Beleuchtungen 16 M.K.

1 bedeutet gekrümmt nach der Lichtquelle der ersten Beleuchtung.

2 bedeutet gekrümmt nach der Lichtquelle der zweiten Beleuchtung.

2 bedeutet erst gekrümmt nach der Lichtquelle der ersten später nach derjenigen der zweiten.

? bedeutet schwach.

Dauer der

Dauer der zweiten Beleuchtung

ersten

Beleuchtung. 10 Sek. 30 Sek. 60 Sek. 90 Sek. 180 Sek. 600 Sek.

\begin{tabular}{r|l|l|l|l|l|l}
\hline 30 Sek. & 1 & 0 & 2 & 2 & 2 & 2 \\
\hline 60 Sek. & 1 & 1 & 2 & 2 & 2 & 2 \\
90 Sek. & 1 & 1 & 2 & 2 & 2 & 2 \\
& & 1 & 1 & 1 & 1 & 1 \\
180 Sek. & 1 & 2 & 2 & 2 & 2 & 2 \\
300 Sek. & 1 & 1 & 1 & 1 & 1 & 2 \\
& 2 & 2 & 2 & 2 & 2 & 2 \\
600 Sek. & 1 & 1 & 1 & 1 & 1 & $1 ?$ \\
& 2 & 2 & 2 & 2 & 2 & 2
\end{tabular}

Die Tabelle 19 ist mit der Tabelle $17 \mathrm{zu}$ vergleichen. Auch hier ist die auf einander folgende Beleuchtung erreicht indem die Pflanzen am Ende der ersten Beleuchtung $180^{\circ}$ gedreht wurden. Als Lichtquelle ist hier aber eine Nernstlampe benutzt worden, sodasz die Resultate 
nicht quantitativ mit den Tabellen 17 und 18 verglichen werden können. Bei diesen Beobachtungen hat man daran zu denken, dasz sie nicht auf dem Klinostaten kontrolliert worden sind. Es liegt aber keine Veranlassung vor an den in der Tabelle angegebenen Krümmungen im Sinne der zweiten Beleuchtung zu zweifeln da die Krümmungen im Sinne der ersten Beleuchtung sehr schwach und diejenigen in der Richtung der zweiten Beleuchtung sehr stark waren und $z u$ früh auftraten um als geotropische Gegenkrümmungen betrachtet werden zu können. Die Ergebnisse dieser Tabelle schlieszen sich ganz denen der Tabellen 17 und 18 an. Nur treten die nach der Lichtquelle der ersten Beleuchtung gerichteten Krümmungen deutlicher auf, wahrscheinlich durch die stärkere Intensität und durch die leichtere Sichtbarkeit einer schwachen Krümmung, wenn die Pflanzen nicht auf dem Klinostaten rotieren.

Aus der Vergleichung dieser Tabelle mit der vorhergehenden geht hervor dasz das geschieden auftreten der zwei induzierten Krümmungsneigungen nicht nur von der Intensität der Beleuchtung sondern auch von der Zeit abhängt, welche zwischen dem Anfange der ersten und dem der zweiten Beleuchtung verläuft. Da diese Beobachtungen nicht auf dem Klinostaten kontrolliert worden sind, kann Tabelle 19 keine Data verschaffen über die Frage ob auch noch negative Krümmungen hinsichtlich der zweiten Beleuchtung sichtbar werden.

Die Erscheinungen, welche bei auf einander folgenden Beleuchtungen auftreten, zeigen sich also ziemlich kompliziert. Um einzelne wichtige Sachen näher zu beleuchten, können hier noch einige Versuche besprochen werden bei welchen zwischen den zwei Reizungen eine gewisse Zeit verläuft, indem der Reiz selber in kurzer Zeit zugeführt worden ist. (Tab. 20).

Führt man 105 M.K.S. d. i. eine Menge welche eine positive Krümmung gibt, einseitig $z u$ und sogleich danach 


\section{Tabelle 20.}

105 M.K.S. (7 Sek.) sogleich danach die entgegengesetzte Seite 105 M.K.S. (7 Sek.)

Zeit zwischen den 2 Reizen. 1 Min.

Keine Krümmung

2 Min. erst Spitze 1 dann Spitze 2

4 Min. erst 1 dann schwach 2

8 Min. erst 1 dann stark 2

an der entgegengesetzten Seite dieselbe Menge, dann entsteht keine sichtbare Reaktion. Wie aber aus der Tabelle hervorgeht, werden die Krümmungen geschieden sichtbar, wenn zwei Minuten zwischen dem Zuführen der zwei Reize verlaufen; zunächst eine Krümmung im Sinne der ersten Beleuchtung und später eine im Sinne der zweiten.

Die Erscheinungen bei Gegenwirkung einer groszen Energiemenge welche eine deutliche negative Spitzen krümmung verursacht und einer schwachen, welche eine positive gibt, gehen noch deutlicher hervor aus einem Versuche wobei 4500 M.K.S. mit 500 M.K.S. kombiniert worden sind. 4500 M.K.S. allein (in 10 Sekunden zugeführt) gibt eine positive Krümmung gefolgt von einer deutlichen negativen Spitzenkrümmung; 500 M.K.S. allein eine starke positive Krümmung. Wird nun erst 500 M.K.S. und 5 Minuten später von der entgegengesetzten Seite 4500 M.K.S. zugeführt, so tritt nur die positive Krümmung von 4500 M.K.S. auf, also eine Krümmung im Sinne der zweiten Beleuchtung. Wird aber erst 4500 M.K.S. und 5 Minuten später 500 M.K.S. zugeführt, so tritt zuerst eine Krümmung im Sinne der ersten Beleuchtung auf und später eine in der Richtung der zweiten.

Von dieser letzten Krümmung ist also nicht festzustellen ob es eine positive durch 500 M.K.S. verursachte Krümmung ist oder eine negative hinsichtlich der 4500 M.K.S. oder ein Zusammenwirken beider.

Die hier mitgeteilten Beobachtungen ermöglichen es 
noch nicht diese Erscheinungen ganz befriedigend zu analysieren. Dafür ist eine gröszere Reihe Beobachtungen nötig, wobei es erwünscht wäre dem ganzen Krümmungsverlauf in der in $\S 4$ beschriebenen Weise auf dem intermittierenden Klinostaten zu folgen.

In diesem Augenblicke kann nur festgestellt werden, dasz bei aufeinander folgender Beleuchtung einer Pflanze von zwei entgegengesetzten Seiten die durch die zweite Beleuchtung induzierte positive Krümmung sich stärker äuszern kann als die durch die erste Beleuchtung induzierte positive Krümmung. Eine negative Krümmung hinsichtlich der zweiten Beleuchtung wird aber durch eine vorhergehende Beleuchtung der anderen Seite von gewisser Dauer unterdrückt.

Ueber dergleichen zweiseitige aufeinanderfolgende Beleuchtungen sind auch von $\mathrm{Clark}$ Versuche mitgeteilt worden. (1. c. Fig. 7). Seine Ergebnisse sind nicht mit den hier gegebenen im Widerspruch. Nur teilt er nicht das Auftreten von Krümmungen im Sinne der ersten Beleuchtung mit. Es liegt aber keine Veranlassung vor $\mathrm{Clark} z \mathrm{u}$ folgen, wenn er von einer Tendenz zu negativen Krümmungen spricht. Wie schon viele Male auseinander gesetzt worden ist, ist es unmöglich festzustellen ob eine Krümmung gerichtet nach der Lichtquelle der zweiten Beleuchtung positiv hinsichtlich der zweiten oder negativ hinsichtlich der ersten Reizung ist. Es ist aber wahrscheinlich, dasz in vielen Fällen die Krümmung nach der Lichtquelle der zweiten Beleuchtung nur von der von dieser Seite zugeführten Energiemenge verursacht wird.

§27. Gleichzeitige Beleuchtung von zwei entgegengesetzten Seiten, der eine einseitige folgt oder vorhergeht.

Im vorigen Paragraphen sind Beleuchtungen besprochen 
worden, welche gleichzeitig oder ganz nach einander stattfanden. Es ist auch möglich, dasz sie nur für einen Teil gleichzeitig stattfinden. Die Fälle welche davon hier besprochen werden müssen sind eine gleichzeitige gleich starke Beleuchtung von zwei entgegengesetzten Seiten, der eine einseitige folgt oder hervorgeht. Die zwei Tabellen. welche diese Beobachtungen enthalten sind mit einander vollkommen zu vergleichen (dieselbe Lichtquelle und Vorgeschichte) wahrscheinlich auch mit den schon besprochenen einseitigen und zweiseitigen Beleuchtungen wobei Glühlampen von 10 Volt als Lichtquelle benutzt worden sind.

In der Mitte zwischen den Lichtquellen in einer Entfernung von $90 \mathrm{cM}$. von links bis auf $90 \mathrm{cM}$. von rechts wurde ein Gefäsz von $20 \mathrm{cM}$. Länge mit Keimlingen aufgestellt. Es wurde also nur die sich in der Mitte des Behälters befindende Pflanze von beiden Seiten ebenso stark beleuchtet. Da aber alle Keimlinge auf dieselbe Weise reagierten kann die Reaktion des sich genau in der Mitte befindenden Keimlings mit Sicherheit festgestellt werden. Alle Pflanzen kamen nach der Beleuchtung auf den Klinostaten.

Die Tabellen 21 und 22 sind so eingerichtet worden dasz man sie leicht mit einander vergleichen kann. Die sehr auffallenden Unterschiede müssen dadurch verursacht werden, dasz bei der Tabelle 21 die einseitige Beleuch tung am Ende, bei Tabelle 22 vor dem Anfange der zweiseitigen Beleuchtung stattfand; d. h. bei der Tabelle 21 fangen die zwei Beleuchtungen gleichzeitig an, bei der Tabelle 22 ist eine der Seiten, welche gleichzeitig nachbeleuchtet werden schon einseitig vorbeleuchtet worden. Dieser Unterschied äuszert sich insbesondere darin, dasz in der Tabelle 22 eine Tendenz besteht dasz die Krümmungen gesondert auftreten, wobei die zuerst induzierte Krümmung auch zuerst sichtbar wird und nach einiger Zeit in eine entgegengesetzt gerichtete Krümmung übergeht. 
Tabelle 21 .

Eine $z$ weiseitige Beleuchtung der eine einseitige folgt,

Bei der zweiseitigen Beleuchtung ist ein Gefäsz mit Keimlingen $z$ wischen den gleich starken Lichtquellen $(5$ H.K.) aufgestellt. In der Mitte beträgt die Beleuchtungsstärke 5 M.K.

$\mathbf{k}=$ gekrümmt nach der Lichtquelle womit nicht nachbeleuchtet wird (kurzdauernde Beleuchtung).

$l=$ gekrümmt nach der Lichtquelle womit auch nachbeleuchtet wird (langdauernde Beleuchtung).

$\begin{aligned} & \mathbf{k} \\ & \mathbf{k}\end{aligned}=$ erst gekrümmt nach der Lichtquelle der langdauernden später nach derjenigen der kurzdauernden Beleuchtung.

Dauer der Einseitige Nachbeleuchtung.

zweiseitigen Bel.

30 Sek.

1 Min.

2 Min.

4 Min.

8 Min.

10 Sek. deutl. $l$ deutl. $l$ deutl. $l$ deutl. $l$

$l$

mit Spitze k

30 Sek. deutl. $l$

deutl. $l$

deutl. $l$ deutl. $l$

mit Spitze k

1 Min. schwach $l \quad l \quad$ deutl. $l$

deutl. $l$

mit Spitze k mit Spitze k

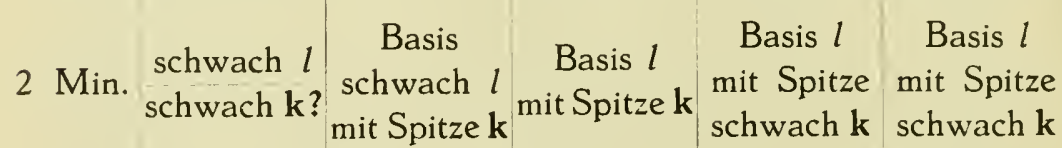

3 Min. $\begin{array}{lc}\text { schwach } l & l \\ \text { schwach } \mathbf{k} & \text { schwach } \mathbf{k}\end{array}$

\begin{tabular}{ccc|c}
5 Min. & $l$ & Basis $l$ & Basis $l$ \\
& $\mathbf{k} ?$ & Spitze $\mathbf{k}$ ? & $\begin{array}{l}\text { Spitze } \\
\text { gerade }\end{array}$
\end{tabular} 
Tabelle 22.

Eine $z$ weiseitige Beleuchtung der eine einseitige vorhergeht.

Bei der zweiseitigen Beleuchtung ist ein Gefäsz mit Keimlingen zwischen den gleich starken Lichtquellen ( 5 H.K.) aufgestellt. In der Mitte beträgt die Beleuchtungsstärke 5 M.K.

$\mathbf{k}=$ gekrümmt nach der Lichtquelle womit nicht vorbeleuchtet wird (kurzdauernde Beleuchtung).

$l=$ gekrümmt nach der Lichtquelle womit erst vorbeleuchtet wird (langdauernde Beleuchtung).

$l$
$\mathbf{k}$ später nach derjenigen der kurzdauernden Beleuchtung.

\begin{tabular}{|c|c|c|c|c|c|}
\hline \multirow{2}{*}{$\begin{array}{c}\text { Dauer der } \\
\text { zweiseitigen } \\
\text { Bel. }\end{array}$} & \multicolumn{5}{|c|}{ Einseitige Vorbeleuchtung. } \\
\hline & 30 Sek. & 1 Min. & 2 Min. & 4 Min. & 8 Min. \\
\hline 10 Sek. & $l$ & $l$ & $l$ & $l$ & $\frac{l}{\text { Spitze }} \mathbf{k}$ \\
\hline 30 Sek. & $l$ & $l$ & $\begin{array}{c}l \\
\text { Spitze } \mathbf{k}\end{array}$ & $\begin{array}{c}l \\
\text { Spitze } \mathbf{k}\end{array}$ & $\begin{array}{l}l \\
\mathrm{k}\end{array}$ \\
\hline 1 Min. & $l$ & $\frac{l}{\text { Spitze } \mathbf{k}}$ & $\begin{array}{l}\text { schwach } l \\
\text { Spitze k }\end{array}$ & $\frac{l}{\text { Spitze } \mathbf{k}}$ & $\begin{array}{l}l \\
\mathbf{k}\end{array}$ \\
\hline 2 Min. & $\begin{array}{l}\mathrm{l} \\
\mathrm{k} ?\end{array}$ & $\begin{array}{l}\text { schwach } \\
\text { Spitze k }\end{array}$ & $\begin{array}{l}\text { schwach } l \\
\text { Spitze } \mathbf{k}\end{array}$ & $\frac{l}{\text { stark k }}$ & $\frac{l}{\text { stark } \mathbf{k}}$ \\
\hline 3 Min. & $\begin{array}{l}l \\
\mathbf{k}\end{array}$ & $\begin{array}{c}\text { gerade } \\
\text { Spitze k }\end{array}$ & $\begin{array}{c}\text { schwach } l \\
\mathbf{k}\end{array}$ & $\begin{array}{c}l \\
\text { stark } \mathbf{k}\end{array}$ & $\frac{l}{\text { stark k }}$ \\
\hline 5 Min. & $\frac{l ?}{\text { Spitze } \mathrm{k}}$ & $\begin{array}{c}\text { gerade } \\
\text { Spitze k }\end{array}$ & $\frac{\text { schwach } l}{\mathbf{k}}$ & $\begin{array}{c}l \\
\text { stark k }\end{array}$ & $\frac{l}{\text { stark k }}$ \\
\hline
\end{tabular}


Bei einer einseitigen Vorbeleuchtung von 60 Sekunden tritt immer erst eine Krümmung im Sinne der längerdauerden Beleuchtung auf und später eine im Sinne der kürzerdauernden schwächeren, welche auch später angefangen hat. Bei allen längeren einseitigen Vorbeleuchtungen der Tabelle 22 tritt immer die Krümmung im Sinne der kürzerdauernden Beleuchtungen auf. Dieser Unterschied zwischen den Tabellen 21 und 22 ist sehr gut $z u$ verstehen, wenn man sich die Data, welche in den vorhergehenden Paragraphen erhalten sind, erinnert. Wie bei einer aufeinanderfolgenden Beleuchtung von zwei entgegengesetzten Seiten die zweite Beleuchtung sich immer äuszert, auch wenn sie schwächer ist als die erste, so auch hier; aber auch darin besteht die Uebereinstimmung dasz es bezüglich dieser Krümmung im Sinne der kürzerdauernden Beleuchtung ganz unmöglich ist festzustellen ob sie vielleicht für einen 'Teil auf einer negativen Krümmung hinsichtlich per längerdauernden Beleuchtung beruht.

Er ist schwieriger einige in der Tabelle 21 gefundene Werte verständlich zu machen. Auch hier treten Krümmungen im Sinne der kürzerdauernden Beleuchtung auf, fast immer sind es äuszerst schwache Spitzenasymmetrieen. Es ist hier nicht möglich zu bestimmen ob diese Krümmungen als positive durch die kürzerdauernde Beleuchtung oder als negative durch die längerdauernde verursacht werden. Gegen diese letzte Möglichkeit spricht das Auftreten dieser Krümmungen bei relativ kleinen Energiemengen der längerdauernden Beleuchtung, kleiner als 2000 M.K.S. welche als einseitiger Reiz nie zu einer negativen Krümmung führen könnten.

Wenn man sieht dasz sowohl in der Tabelle 21 als in der Tabelle 22 bei einer zweiseitigen Beleuchtung, welche zwei Minuten dauert, Krümmungen im Sinne der kürzerdauernden Beleuchtung auftreten dann möchte man erwarten 
dasz auch bei einer zweiseitigen gleichzeitigen Beleuchtung bei Beleuchtung der entgegengesetzten Seiten mit diesen selben Energiemengen eine Spitzenkrümmung im Sinne der schwächeren Beleuchtung auftreten würde. Schon in $\S 25$ ist diese Möglichkeit besprochen worden und mitgeteilt dasz das Vorkommen dieser Krümmungen auf Grund der bisher bekommenen Data nicht verneint und nicht bestätigt werden kann. Wenn sie wirklich vorkommen, wenn es also möglich ist durch Zusammenwirkung einer Lichtmenge welche eine Krümmung gibt welche über die gröszte maximale Krümmungsstärke hin ist und einer welche maximal oder fast maximal ist, eine Krümmung nach der schwächsten Beleuchtung zu bekommen dann würden sich diese Erscheinungen über zweiseitige Beleuchtung auf befriedigende Weise erklären lassen. Man wird aber ausführlichere Untersuchungen abwarten müssen um diese Probleme zu einer Lösung zu bringen. Es musz hier noch eine Erscheinung besprochen werden. In der Tabelle 21 kommen bei einer Dauer der gleichzeitigen Beleuchtung von 3 und 5 Minuten und bei einer einseitigen Nachbeleuchtung von 2, 4 und 8 Minuten keine negative Krümmungen hinsichtlich der längerdauernden Beleuchtung vor. Auch für diese Erscheinung ist eine Parallele bei den auf einander folgenden Beleuchtungen von zwei entgegengesetzten Seiten zu finden. Hier kommt eine negative Krümmung hinsichtlich der zweiten Beleuchtung nach einer vorhergehenden Beleuchtung von 3 Minuten und länger der entgegengesetzten Seite nicht mehr vor. Es ist sehr wahrscheinlich, dasz obwohl die zwei Beleuchtungen in der Tabelle 21 teils ineinander fallen, dennoch das nicht mehr Auftreten einer negativen Krümmung hinsichtlich der längerdauernden Beleuchtung auf derselben Ursache beruht, wie wenn die Beleuchtungen ganz nach einander stattfinden.

In diesem Augenblicke ist es natürlich noch nicht mög- 
lich mit den wenigen erhaltenen Data diese Prozesse ganz zu analysieren. Es ist aber sehr wahrscheinlich, wie oben zu zeigen versucht worden ist, dasz all diese Kombinationen zweiseitiger Beleuchtungen als Zusammenwirkung zweier einseitiger betrachtet werden müssen. 


\section{ABSCHNITT VI. \\ Allseitige Beleuchtungen.}

\section{$\S 28$. Einleitung.}

Ehe zur Beschreibung der Versuche und zu der Betrachtung der erhaltenen Resultate übergegangen werden kann musz hier erst umschrieben werden was man unter einer allseitigen Beleuchtung zu verstehen hat. Obwohl dieser Ausdruck nicht ganz deutlich und auch nicht ganz genau ist so wird er doch da er bei diesen Versuchen immer für dieselbe Beleuchtungsweise benutzt worden ist, keine Veranlassung zu Miszverständnissen geben können. Unter allseitiger Beleuchtung hat man nicht eine gleichzeitige Beleuchtung aller Seiten einer Pflanze zu verstehen sondern eine aufeinanderfolgende Beleuchtung indem sie um ihre eigene Achse vor einer feststehenden Lichtquelle gedreht wird. Es ist also nicht was Pfeffer unter einer diffusen Beleuchtung versteht, sondern genau derselbe Begriff womit auch $\mathrm{Pring}$ sheim und $\mathrm{Clark}$ gearbeite haben. Versuche mit gleichzeitiger Beleuchtung aller Seiten, sind nicht ausgeführt worden. ${ }^{1)}$ Ebensowenig sind die Pflanzen je von oben beleuchtet worden.

1) Es hat sich gezeigt, dasz ein prinzipieller Unterschied zwischen einer diffusen und einer allseitiger aufeinanderfolgender Beleuchtung nicht besteht. 
$\S 29$. Methode.

Alle Versuche haben stattgefunden im Dunkelzimmer des Gewächshauses bei $23^{\circ}$ Cels., indem als Lichtquelle eine Nernstprojektionslampe benutzt worden ist. Die allseitige Beleuchtung wurde durch einen speziell dazu gebauten Apparat hergestellt. Es ist eine Art Multiklinostat, wobei ein durch ein Gewicht getriebenes Uhrwerk eine Hauptachse drehen läszt. Ebenso wie beim bekannten Pfefferschen Klinostaten wird die Bewegung durch einen Flügel reguliert. Die Bewegung der Hauptachse wird auf 20 vertikal gestellte Nebenachsen übergebracht. Auf jeder dieser Nebenachsen befindet sich ein Tischchen, worauf ein Topf mit einer Pflanze gestellt werden kann. Alle Keimlinge drehen also mit derselben Geschwindigkeit. Durch Aenderung des Flügelregulators und der Grösze des Gewichtes ist die Umdrehungszeit zwischen 4 Sekunden und 4 Minuten zu variieren. Die Tischchen, worauf sich die Töpfe befinden, sind so gestellt worden, dasz bei einer Entfernung des Apparates von der Lichtquelle von 1 Meter. die Pflanzen, welche in den hinteren Reihen stehen, keinen Schatten von den vorderen bekommen können. Die Pflanzen werden immer genau eine ganze Anzahl Drehungen beleuchtet. Dieses ist durch eine an der Hauptachse sich befindende elektrische Kontaktvorrichtung erreicht. Wenn diese einen Strom schlieszt und dadurch ein Zeichen gegeben wird, wird mit der Beleuchtung angefangen oder dieselbe beendet. Bei einer Versuchsreihe wurde durch den Strom in dem Augenblicke, da dieser durch die Kontaktvorrichtung geschlossen wurde, automatisch der Lichtverschlusz geöffnet oder geschlossen. Es ist übrigens durch das Einschieben eines Sperrkegels möglich gemacht, dasz der Apparat in dem Augenblicke hält, worauf das Zeichen gegeben wird, dasz die 0 Lage erreicht ist. Auf diese Weise ist zu Stande gebracht, dasz wenn nach einer 
Rotation die. Beleuchtung einseitig fortgesetzt wird, alle Pflanzen von einer bestimmten Seite, senkrecht auf der Nutationsebene, beleuchtet werden. Die Drehung ist physiologisch und physisch kontrolliert worden; auch unter den günstigsten Umständen waŕ sie nicht ganz regelmäszig; die Ungenauigkeiten waren aber zu klein um bei den längstdauernden Beleuchtungen womit hier experimentiert wurde zu Krümmungen zu führen. Da der Apparat nicht sogleich mit der ganzen Geschwindigkeit drehte, wurde mit der Beleuchtung erst angefangen, wenn eine konstante Geschwindigkeit erhalten war. In jedem der 20 Töpfe befand sich genau in der Mitte ein Keimling. Es ist also bei dieser Experimentierweise eine grosze Kultur nötig, nicht so sehr weil auf diese Weise so viel Keimlinge benutzt werden, als weil eine sehr grosze Anzahl von Töpfen bearbeitet werden musz (etwa 200 pro Tag) um immer einen Vorrat benutzbares Material zu haben. Die Methode, mittels welcher der Einflusz einer allseitigen Vorbeleuchtung untersucht worden ist, stimmt prinzipiell ganz mit derjenigen Pringsheims überein, welcher auch Clark gefolgt ist. Die hier benutzte Methode weicht aber in einigen Punkten von derjenigen $\mathrm{Pr}$ ing sheims ab. Pringsheim und Clark planzten viele Keimlinge in einen Topf. Das hat die Beschwerde, dasz die Pflanzen nicht mehr um ihre eigene Achse drehen, wodurch sie sich nicht immer in derselben Entfernung von der Lichtquelle befinden und ungleichmäszig beleuchtet werden. Sie müssen auch notwendig in einanders Schatten kommen. Bei dem hier benutzten Multiklinostaten wo jeder Keimling um seine eigene Achse dreht ist diesen Fehlern vorgebeugt worden. Pringsheim klagt über die schlechten Resultate bei seinen Versuchen (l. c. 2 Mitt. p. 438). „Eine andere gröszere Schwierigkeit lag darin, dasz das Material das bisher so schön übereinstimmende Werte gegeben hatte sich bei diesen neuen Versuchen 
als auszerordentlich launisch erwies, so dasz auch unter Zuhilfenahme mehrerer Einzelbestimmungen keine ganz befriedigende Uebereinstimmung erhalten werden konnte."

Clark erwähnt diese Beschwerden nicht. Wichtiger ist der Unterschied in der Weise, worauf bei ihnen und bei diesen Versuchen einseitig nachbeleuchtet worden ist. Sie haben stets nachbeleuchtet mit derselben Intensität womit auch die Vorbeleuchtung stattfand. Pringsheim zweifelte ob die von ihm benutzte Methode wohl die richtige sei. "Vielleicht wäre es doch besser gewesen die Präsentationszeiten bei einer höheren Lichtintensität zu messen als sie der Vorbeleuchtung diente."

Bei den Versuchen, welche in diesem Abschnitte betrachtet werden, hat die Nachbeleuchtung in möglichst kurzer Zeit stattgefunden.

Das hat sich ais ein Vorteil erwiesen.

$1^{0}$. da es nur auf diese Weise möglich ist die Nachbeleuchtung immer auf dieselbe Weise zuzuführen; dadurch ist erreicht, dasz die Resultate der verschiedenen Intensitäten womit vorbeleuchtet wurde bei einer gleichförmigen Nachbeleuchtung verglichen werden.

$2^{0}$. da es sich gezeigt hat, dasz der Zustand, welcher am Ende der Vorbeleuchtung besteht, sich sehr schnell ändert, so dasz es einen Unterschied machen musz ob die Nachbeleuchtung mit einer selben Energiemenge in kurzer oder in langer Zeit stattfindet.

$3^{0}$. da eine Anpassung an eine bestimmte Intensität nicht besteht, weshalb es keinen $Z$ weck hat die Vorbeleuchtung und die Nachbeleuchtung mit derselben Intensität geschehen $z$ u lassen.

$4^{0}$. da wenn mit sehr schwacher Intensität vorbeleuchtet wird es bei der einseitigen Nachbeleuchtung mit derselben Intensität unmöglich sein würde negative Krümmungen zu bekommen, indem gerade das Studieren der negativen Erscheinungen das Wichtigste ist. 
Es ist möglich gemacht worden die einseitige Beleuchtung auch mit anderen Intensitäten als womit die allseitige Vorbeleuchtung stattfand gleich nach dem Ende der Rotation anfangen zu lassen. Das ist in der einfachsten Weise erreicht durch Benutzung von Milch- und Mattglasscheiben welche vor dem Lichtverschlusse befestigt werden konnten. Die Verwechslung dieser Scheiben kostete nicht mehr Zeit als 5 Sekunden.

Im Anfange haben bei jedem Versuche immer Kontrollbeobachtungen stattgefunden, indem einige wenige Pflanzen vor der einseitigen Nachbeleuchtung weggenommen und in das Dunkel gestellt wurden. Als es sich aber gezeigt hatte, dasz jede Sekunde welche zwischen Vor- und Nachbeleuchtung gespart werden konnte eine gröszere Genauigkeit der Resultate möglich machte, sind diese Kontrollversuche unterblieben. Im allgemeinen waren die Resultate sehr regelmäszig d.h. alle Pflanzen eines Versuches reagierten auf dieselbe Weise, so dasz das Unterlassen dieser Kontrollversuche nicht schwer $z u$ wiegen hat. In einigen Fällen, welche später noch zu erwähnen sind, war die Reaktion nicht bei allen Pflanzen dieselbe; einige reagierten positiv, andere negativ oder gar nicht. Diese Beobachtungen sind mit einen ? versehen. Durch Vergleichung mit den Resultaten bei zweiseitigen Beleuchtungen ist Veranlassung da, zu denken dasz in diesen Fällen nicht eine unregelmäszige Drehung des Klinostaten die Ursache dieser Erscheinung ist, sondern die speziellen Umstände der Beleuchtung. Bei den Versuchen, wobei die Stärke der Reaktion gemessen wurde, (Tabelle 28 und 32) ist dem Einflusse der Temperatur besondere Sorge gewidmet. Indem der ganze Apparat in einem groszen Heukasten eingeschlossen wurde war es möglich die Temperatur während des Versuches auf $0,1^{\circ}$ C. konstant zu halten. Bei dieser Serie waren die Pflanzen im Dunkelzimmer bei konstanter Temperatur gezogen worden und eine Stunde 
vor der Beleuchtung in den Apparat gebracht bei derselben noch genauer regulierten Temperatur. Bei den anderen Versuchen betrugen die Temperaturwechslungen nicht mehr als $1^{\circ}$ Cels.

§30. Eine allseitige Beleuchtung, der eine einseitige folgt.

Ueber den Einflusz einer allseitigen Beleuchtung sind viele Versuche angestellt worden, wovon hier nur zwei Serien mitgeteilt werden. Die eine gibt eine qualitative Uebersicht, wie unter dem Einflusse einer allseitigen Vorbeleuchtung das Reaktionsvermögen auf eine einseitige Nachbeleuchtung sowohl für die positive als für die negative Reaktion geändert wird. Die zweite Serie gibt mit gröszerer Genauigkeit, wie das Reaktionsvermögen für die positive Krümmung durch Vorbeleuchtung geringer wird.

Diesen Versuchen mit allseitiger Vorbeleuchtung ist sehr viel Zeit und Mühe gespendet worden. Es hat sich aber gezeigt, dasz das Problem, welches durch diese Versuche $z \mathfrak{u}$ lösen war, ein Scheinproblem ist, dasz es jedenfalls eine viel zu komplizierte und unnötig schwierige Experimentierweise notwendig machte. Es ist eine weit verbreitete Idee, dasz die Planzen unter dem Einflusse des Lichtes eine Aenderung ihrer Empfindlichkeit erleiden würden. Dieses würde sich in einer Abnahme des Reaktions vermögens vorbeleuchteter Pflanzen äuszern. Das schwächere Reaktionsvermögen wurde einer geänderten Stimmung der Pflanze zugeschrieben. Wie sich aber aus der Besprechung der erhaltenen Resultate und der Vergleichung mit den im vorigen Abschnitte studierten zweiseitigen Beleuchtungen ergeben wird, macht es keinen prinzipiellen Unterschied ob eine Pflanze allseitig oder zweiseitig vorbeleuchtet worden ist. Eine zweiseitige Beleuchtung kann 
aber viel genauer untersucht werden, da hier die Lichtmengen, welche den beiden Seiten zugeführt worden sind, vollkommen bekannt sind und da mit viel gröszerer Sicherheit untersucht werden kann, ob bei Kombination dieser zwei einseitigen Beleuchtungen neue Erscheinungen auftreten und ob wirklich Veranlassung da ist von einer Lichtstimmung der Pflanzen zu reden. Nur bei genügender Kenntnis von der Weise wie entgegengesetzt gerichtete Reize zusammenwirken, würde die Analyse der viel verwickelteren Prozesse, welche in diesem Abschnitte besprochen werden, zu Ende geführt werden können. Jetzt ist es nur möglich darauf hinzuweisen, dasz dieselben Prinzipien, welche der Kombination einseitiger Beleuchtungen zu zweiseitigen zu Grunde liegen, bei den allseitigen Beleuchtungen zurückgefunden werden können.

Als Intensitäten für die allseitigen Vorbeleuchtungen sind aus experimentellen Gründen gewählt worden 5,5 M.K., 12,1 M.K., 25 M.K., 100 M.K. und 450 M.K. Die Nachbeleuchtung hat stattgefunden mit 9 verschiedenen zwischen 22 M.K.S. und 27000 M.K.S. wechselnden Energiemengen. Da diese Energiemengen bei allen Tabellen auf dieselbe Weise erhalten sind, ist es gestattet die Resultate dieser Tabellen mit einander $z u$ vergleichen. In der Tabelle 23 ist der Resultat der Energiemengen, womit die einseitige Beleuchtung immer stattgefunden hat, aufgenommen. Von 22 M.K.S. bis 1000 M.K.S. tritt eine starke positive Krümmung auf, bei 4500 M.K.S. eine positive Krümmung, welche in eine negative übergeht, und bei 13500 M.K.S. und 27000 M.K.S. eine deutliche negative Spitzenkrümmung. Bei den Tabellen 24 bis 27 musz daran gedacht werden, dasz die Beobachtungen nicht alle auf dem Klinostaten kontrolliert worden sind. Es ist also nicht ausgeschlossen, dasz dort wo zunächst eine deutliche positive Krümmung beobachtet worden ist und später eine entgegengesetzt gerichtete, diese negative 
teilweise auf der Gegenwirkung der Schwerkraft beruhen kann. Diese Beobachtungen sind später nicht auf dem Klinostaten wiederholt worden, da auf Grund der Versuchsprotokolle (Zeit worauf die negative Reaktion sichtbar wird und Stärke der vorhergehenden Krümmung) die Möglichkeit als ausgeschlossen betrachtet werden musz, dasz es nur geotropische Gegenkrümmungen gewesen sein sollten. Einige wichtige Punkte, wie die negativen Krümmungen in der Tabelle 25 bei 3 Minuten Vorbeleuchtung und in der Tabelle 26 bei 100 Sekunden sind wohl auf dem Klinostaten kontrolliert worden.

\section{Zeichenerklärung.}

+ alle Pflanzen deutlich positiv gekrümmt.

++ alle Pflanzen stark positiv gekrümmt.

0 alle Pflanzen nicht gekrümmt.

- alle Pflanzen deutlich negativ gekrümmt.

+ ? wenige Pflanzen schwach positiv gekrümmt.

- ? wenige Pflanzen schwach negativ gekrümmt.

Zwei Zeichen untereinander bedeutet dasz die Reaktion nach einer Stunde mit dem ersten nach 2 Stunden mit dem zweiten Zeichen übereinstimmt.

$$
\text { Tabelle } 23 .
$$

$\mathrm{Nicht}$ vorbeleuchtet

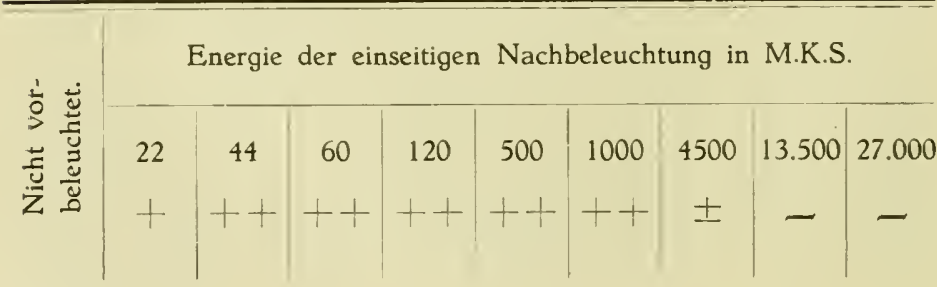


Tabelle 24.

Intensität der allseitigen Vorbeleuchtung 5.5 M.K.

\begin{tabular}{|c|c|c|c|c|c|c|c|c|c|}
\hline \multirow{3}{*}{ 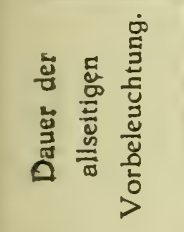 } & \multicolumn{9}{|c|}{ Energie der einseitigen Nachbeleuchtung in M.K.S. } \\
\hline & $4 \times 5$ & $8 \times 5.5$ & $5 \times 12$ & $10 \times 12$ & $5 \times 100$ & $10 \times 10$ & $10 \times 45$ & $30 \times 45$ & $60 \times 450$ \\
\hline & 22 & 44 & 60 & 120 & 500 & 1000 & 4500 & 13.500 & 27.000 \\
\hline 10 Sek. & + & ++ & ++ & ++ & $+t$ & ++ & \pm & - & - \\
\hline 100 Sek. & & 0 & $+?$ & + & ++ & $+t$ & \pm & - & - \\
\hline $3 \mathrm{Min}$. & & & $+?$ & + & ++ & $+\frac{1}{1}$ & \pm & - & - \\
\hline $5 \mathrm{Min}$. & & 0 & 0 & + & ++ & ++ & + & 一? & $-?$ \\
\hline 20 Min. & & & & + & ++ & ++ & + & + & + ? \\
\hline 1 Stunde & & & 0 & + & ++ & ++ & ++ & + & 0 \\
\hline
\end{tabular}

Tabelle 25 .

Intensität der allseitigen Vorbeleuchtung 5,5 M.K.

\begin{tabular}{c|c|c|c|c|c|c|c|c|c}
\hline \multirow{2}{*}{$\begin{array}{c}\text { Dauer der } \\
\text { allseitigen } \\
\text { Vorbeleuchtung. }\end{array}$} & \multicolumn{7}{|c|}{ Energie der einseitigen } & Nachbeleuchtung in M.K.S. \\
\cline { 2 - 8 } & 22 & 44 & 60 & 120 & 500 & 1000 & 4500 & 13.500 & 27.000 \\
\hline 10 Sek. & + & + & ++ & ++ & ++ & ++ & \pm & $\pm ?$ & - \\
36 Sek. & 0 & $+?$ & + & + & ++ & ++ & - & $\pm ?$ & - \\
100 Sek. & 0 & 0 & 0 & $+?$ & ++ & ++ & $\pm ?$ & - & - \\
3 Min. & & & 0 & $?$ & $?$ & \pm & $\pm ?$ & $-?$ & $-?$ \\
5 Min. & & & 0 & 0 & 0 & + & + & $\pm ?$ & $-?$ \\
20 Min. & & & & 0 & + & ++ & ++ & ++ & ++ \\
1 Stunde & & & & $+?$ & ++ & ++ & ++ & ++ & ++
\end{tabular}


Tabelle 26.

Intensität der allseitigen Vorbeleuchtung 25 M.K.

Dauer der allseitigen

Vorbeleuchtung.
Energie der einseitigen Nachbeleuchtung in M.K.S.

22

$44 \quad 60$

120

5001000

4500

\begin{tabular}{|c|c|c|c|c|c|c|c|c|}
\hline 10 Sek. & 0 & $+?$ & + & + & + & \pm & - & - \\
\hline 36 Sek. & & & 0 & $+?$ & $+?$ & - & - & - \\
\hline 100 Sek. & & & 0 & ? & $?$ & -? & -? & - \\
\hline $3 \mathrm{Min}$. & & & 0 & 0 & 0 & -? & - & - \\
\hline $5 \mathrm{Min}$. & & & & 0 & 0 & $+?$ & + & - \\
\hline $20 \mathrm{Min}$. & & & & & & ++ & ++ & +1 \\
\hline 1 Stunde & & & 0 & - & ++ & ++ & +1 & + + \\
\hline
\end{tabular}

Tabelle 27.

Intensität der allseitigen Vorbeleuchtung 100 M.K.

\begin{tabular}{|c|c|c|c|c|c|c|c|c|c|}
\hline \multirow{2}{*}{$\begin{array}{c}\text { Dauer der } \\
\text { allseitigen } \\
\text { Vorbeleuch } \\
\quad \text { tung. }\end{array}$} & \multicolumn{9}{|c|}{ Energie der einseitigen Nachbeleuchtung in M.K.S. } \\
\hline & 22 & 44 & 60 & 120 & 500 & 1000 & 4500 & 13.500 & 27.000 \\
\hline 10 Sek. & 0 & 0 & 0 & $+?$ & + & + & - & - & - \\
\hline 36 Sek. & & & 0 & 0 & 0 & 0 & - & - & - \\
\hline 100 Sek. & & & 0 & 0 & 0 & 0 & - ? & - ? & $-?$ \\
\hline 5 Min. & & & & 0 & 0 & 0 & 0 & $+?$ & ++ \\
\hline 20 Min. & & & & & & 0 & + & + & ++ \\
\hline 1 Stunde & & & & & & + & +1 & ++ & $1++$ \\
\hline
\end{tabular}


Es ist insbesondere die Betrachtung der Tabelle 24 wo die Intensität der allseitigen Vorbeleuchtung am schwächsten ist, dasz man sich am leichtesten über dem Einflusse einer allseitigen Vorbeleuchtung orientieren kann. Wenn man die sechs ersten vertikalen Kolumnen dieser Tabelle betrachtet, wobei die Stärke der einseitigen Nachbeleuchtung von $22 \mathrm{zu} 1000$ M.K.S beträgt, dann sieht man, dasz nach einer Vorbeleuchtung von 100 Sekunden schon mit 60 M.K.S. nachbeleuchtet werden musz um eine Krümmung zu bekommen indem nach 10 Sekunden Vorbeleuchtung noch 22 M.K.S. genügten. Bei noch längerdauernden Vorbeleuchtungen braucht nicht viel mehr Energie zugeführt zu werden. Auch nach Rotation während einer Stunde bei einer Intensität von 5,5 M.K. gibt 120 M.K.S. eine deutliche positive Krümmung. Wenn man nun auch bei den Tabellen 25 und 26 die 6 ersten vertikalen Kolumnen betrachtet, dann zeigt es sich, dasz auch hier eine selbe Erscheinung auftritt. Es wird hier immer schwieriger eine positive Krümmung zu bekommen. Die Erscheinung ist aber viel stärker und teils durch andere Prozesse verdeckt. In der Tabelle 25 sieht man, dasz bei einer Intensität von 12,1 M.K. nach einer Vorbeleuchtung von 100 Sekunden erst 500 M.K.S. eine deutliche positive Krümmung geben. Nach einer Vorbeleuchtung von 3 Minuten ist es gar nicht mehr möglich eine deutliche positive Krümmung zu bekommen. Bei den noch stärkeren Intensitäten von $25 \mathrm{M}$.K. und 100 M.K. tritt dieses Stadium worin gar keine deutliche positive Krümmungen zu bekommen sind, noch früher auf: bei 25 M.K. bei einer Dauer der allseitigen Vorbeleuchtung von 100 Sekunden und bei 100 M.K. von 36 Sekunden. In all diesen Tabellen kann man also die Erscheinung zurückfinden, dasz je nachdem die Pflanzen länger oder stärker vorbeleuchtet werden auch mehr Energie zugetührt werden musz um eine positive Krümmung zu bekommen. Um diese Erscheinung noch sorg- 
fältiger festzustellen, kann hier eine Versuchsreihe besprochen werden, wobei versucht worden ist diese Abnahme der Empfindlichkeit quantitativ zu bestimmen. Dafür ist die Stärke der resultierenden positiven Krümmung bei verschiedener Stärke der einseitigen Nachbeleuchtung $1 \frac{1}{2}$ Stunde nach dem Anfange der Reizung gemessen worden. Bei diesen Beobachtungen wird also die Stärke der erreichten Krümmung in mM. (horizontale Abweichung der Spitze) ausgedrückt (vergl. §8) statt durch ein + oder ein ++ Zeichen wie das in den Tabellen 24 bis 27 geschehen ist.

Wie im Abschnitte IV bei der Betrachtung des Einflusses der Lichtrichtung ist auch hier aus diesen Zahlen die Energiemenge berechnet worden, welche nötig ist um nach $1 \frac{1}{2}$ Stunde eine Krümmung von $1 \mathrm{mM}$. zu bekommen. Diese Bestimmungen haben bei einer Intensität der Vorbeleuchtung von 30 M.K. stattgefunden. Die Reizdauer der einseitigen Nachbeleuchtung ist so kurz wie möglich gewählt worden. Die Tabelle 28 gibt die Resultate.

$$
\text { Tabelle } 28 .
$$

Bestimmung der Energiemenge der Nachbeleuchtung, welche nach $1 \frac{1}{2}$ Stunde eine Krümmung von $1 \mathrm{mM}$. verursacht, wenn während verschiedener Zeitdauer allseitig vorbeleuchtet wird mit $30 \mathrm{M} . \mathrm{K}$ Temp. $23^{\circ} \mathrm{C}$.

Dauer der allseitigen Vorbeleuchtung mit 30 M.K.

Energie der Nachbeleuchtung.

20 Sek.

30 Sek.

1 Min.

2 Min.

4 Min.

15 Min.

30 Min.

4 Stunden
200 M.K.S.

365 M.K.S. 800 M.K.S.

?

2500 M.K.S.

1200 M.K.S.

800 M.K.S.

600 M.K.S. 
Wenn man vorläufig nur die vier ersten dieser Zahlen betrachtet dann zeigt sich auch hier wieder die Abnahme der Empfindlichkeit durch die Vorbeleuchtung. Nach 2 Minuten Vorbeleuchtung ist gar keine positive Krümmung mehr zu bekommen.

In dieser Beobachtungsreihe ist also die schon bekannte Tatsache bestätigt worden, dasz vorbeleuchtete Pflanzen weniger empfindlich sind als völlig im Dunkeln gewachsene. Auch Pringsheim war zu diesem Resultat gekommen. Es ist aber fraglich ob es wohl erwünscht ist von einer geringeren Empfindlichkeit vorbeleuchteter Pflanzen zu reden da das eine Aenderung des Perzeptionsvermögens bedeuten würde. $U_{m}$ die Erscheinungen, welche bei allseitiger Beleuchtung auftreten zu verstehen, musz man sie mit einer zweiseitigen Beleuchtung vergleichen. Man hat dazu das Recht da eine allseitige Beleuchtung als eine Reihe von einseitigen Reizungen betrachtet werden kann. Es ist natürlich nicht zu erwarten, dasz der Resultat derselbe sein wird, wenn mit einer Intensität von $5,5 \mathrm{M} . \mathrm{K}$. z.B. während 10 Sekunden allseitig gereizt wird oder wenn das zweiseitig stattfindet. Bei einer allseitigen Beleuchtung werden allen Seiten der Pflanze zusammen $10 \quad 5,5=55$ M.K.S. zugeführt. Um eine derartige Energiemenge zweiseitig zuzuführen würde man entweder mit derselben Intensität beide Seiten 5 Sekunden oder und, das ist sicher richtiger, während derselben Zeit mit der halben Lichtstärke beleuchten müssen. Will man also untersuchen in wie weit eine allseitige Rotation bei 5,5 M.K. dasselbe Resultat hat als eine zweiseitige Beleuchtung. dann wird man sie mit einer zweizeitigen gleichseitigen Beleuchtung mit der halben Lichtstärke 2,75 M.K. vergleichen müssen. Nun ist bei der zweiseitigen Beleuchtung eine Tabelle (Tab. 21 p. 131) für eine Intensität von 5 M.K. gegeben worden. Es wird sich zeigen, dasz diese wirklich sehr gut mit der hier gegebenen Tabelle für eine allseitige Beleuchtung bei 
12,1 M.K. (Tabelle 25, p. 144) verglichen werden kann.

In der Tabelle 21 sieht man in der ersten vertikalen Kolumne, dasz nach einer zweiseitigen Vorbeleuchtung von 30 Sekunden bei einer einseitigen Nachbeleuchtung während 30 Sekunden, d. i. mit 150 M.K.S., eine deutliche positive Krümmung auftritt; nach einer Vorbeleuchtung von 2 Minuten können keine deutliche Krümmungen mehr $z u$ stande kommen. Bei diesen zweiseitigen Beleuchtungen wird die sogenannte Unempfindlichkeit dadurch verursacht, dasz die entgegengesetzt gerichtete Krümmung überwunden werden musz. Es ist gar nicht wunderbar, dasz der Ueberschusz welcher einer der Seiten gegeben werden musz um eine Krümmung nach dieser Seite zu bekommen gröszer sein musz je nachdem die Reizung der anderen Seite stärker ist.

Die Uebereinstimmung zwischen den Tabellen 21 und 25 ist aber noch gröszer. Wie schon besprochen wurde, treten bei der zweiseitigen Beleuchtung von 2 und 3 Minuten schon bei schwachen einseitigen Nachbeleuchtungen sehr schwache Krümmungen auf, welche von der Lichtquelle womit nachbeleuchtet worden ist hinweggerichtet sind. Etwas derartiges findet man auch in der Tabelle 25 4. horizontale Kolumne. Bei 1000 M.K.S. tritt hier nach der positiven eine schwache negative Krümmung auf, indem auch bei 500 M.K.S. und 120 M.K.S. eine Tendenz zur negativen Reaktion besteht. Die Krümmungen sind hier nicht deutlich; einige Pflanzen eines selben Versuches krümmen sich schwach positiv aber immer sind einige wenige da welche sich von der Lichtquelle hinweg krümmen. Diese selbe Erscheinung sieht man auch in der Tabelle 26 für $25 \mathrm{M}$.K. nach einer Vorbeleuchtung, welche 100 Sekunden dauert, bei Nachbeleuchtung mit 500 und 1000 M.K.S. Clark nennt dies das Empfindlicherwerden für die negative Reaktion. Dieser Name ist ebenso wenig zutreffend wie das schon besprochene Unempfindlicherwerden für die positive Reaktion. 
Aehnlich wie bei einer zweiseitigen Vorbeleuchtung können die hier genannten Erscheinungen erklärt werden, wenn man die Vorbeleuchtung als eine Kombination einseitiger Beleuchtungen auffaszt deren eine während längerer Zeit fortgesetzt wird. Es ist wohl zu verstehen, dasz die Energie der Vorbeleuchtung sich mit der an derselben Seite bei der Nachbeleuchtung zugeführte Energie summiert und so weniger Energie einseitig zugeführt zu werden braucht als ohne Vorbeleuchtung um eine negative Krümmung zu induzieren. Neben dieser echten negativen Krümmung kommt ähnlich wie bei zweiseitiger Vorbeleuchtung $\S 28$ noch eine scheinbare negative Krümmung vor, welche dadurch entsteht, dasz die längerdauernde Beleuchtung einen schwächeren positiven Effekt hat als die kürzerdauernde.

Die Abnahme der Empfindlichkeit für die positive Reaktion und die Zunahme der Empfindlichkeit für die negative als Folge einer Vorbeleuchtung sind also zwei Prozesse, welcheleichterklärt werden können, wenn die allseitige Vorbeleuchtung als ein Aufeinanderfolgenvoneinseitigen betrachtet wird und die Nachbeleuchtung als eine Fortsetzung der Reizung einer der vorbeleuchteten Seiten.

In der Tabelle 24 (p. 144) ist von diesem sogenannten Empfindlicherwerden für die negative Reaktion nichts zu spüren; die Ursache musz wahrscheinlich in der schwachen Intensität gesucht werden. Es ist aber nicht ausgeschlossen, dasz die Erscheinung doch auch hier auftritt dasz aber zufälligerweise gerade die guten Lichtmengen nicht untersucht worden sind. Man könnte sie hier erwarten bei 6 bis 10 Minuten Vorbeleuchten und eben in diesem Gebiete sind keine Versuche angestellt worden. Es ist aber auch möglich, dasz bei dieser schwachen Intensität 
die Erscheinung gar nicht mehr vorkommt. Ein Analogon könnte man hierfür finden in der Tatsache, dasz auch bei schwachen einseitigen Beleuchtungen keine negative Krümmungen auftreten.

Eine dritte Erscheinung zeigt sich, wenn man die drei letzten vertikalen Kolumnen der Tabellen 23-26 betrachtet. Bei kurzer Dauer der Vorbeleuchtung treten bei diesen Energiemengen immer negative Krümmungen auf. Bei längerer Vorbeleuchtung geht aber dieses Vermögen verloren. Diese selbe Erscheinung ist auch bei einer zweiseitigen Beleuchtung mit nachfolgender einseitigen gefunden (Tab. 21) ebenso bei einer aufeinanderfolgenden Beleuchtung von zwei entgegen gesetzten Seiten. ( $\S 26$ ). Es zeigt sich in all diesen Fällen dasz, wenn eine der Seiten vorbeleuchtet worden ist, das Vermögen der anderen Seite um negative Kr ümmungen zu Stande $z u$ bringen abnimmt.

Eine vierte Erscheinung zeigt sich schlieszlich in der Zunahme der Empfindlichkeit für positive Krümmungen nach langer Dauer der Vorbeleuchtung. In der Tabelle 25 wird nach einer Vorbeleuchtung von 5 Minuten bei 1000 M.K.S. eine positive Krümmung sichtbar nach 20 Minuten bei 500 M.K.S. und nach einer Stunde bei etwa 120 M.K.S. So ist auch in den Tabellen 26 und 27 nach 5 Minuten schon wieder die Möglichkeit positive Krümmungen zu bekommen zurückgekehrt. Je länger auch hier die Vorbeleuchtung dauert desto stärker sind die positiven Krümmungen und bei desto geringerer Energiemenge treten sie auf. In der Tabelle 28 ist das quantitativ festgestellt worden. Hier musz nach einer Vorbeleuchtung von 4 Minuten, 2500 M.K.S. nach einer von 4 Stunden nur 600 M.K.S. zugeführt werden um eine Krümmung von $1 \mathrm{mM}$ nach $1 \frac{1}{2}$ Stunde zu bekommen.

Die Tabelle 21 für zweiseitige Beleuchtung mit nachfolgender einseitiger geht nur bis zu einer Beleuchtung 
von 5 Minuten. Auch hier ist aber festgestellt worden, dasz die positiven Krümmungen der zwei letzten vertikalen Kolumnen ( $l$ ) nach einer Vorbeleuchtung von 5 Minuten viel stärker sind als nach einer von 3 Minuten. Dieses Stärkerwerden der positiven Krümmungen wird wahrscheinlich zusammenhangen mit der sub 3 konstatierten Abnahme des negativen Reaktionsvermögens. Man findet es auch zurück in der gleichzeitigen Beleuchtung von zwei entgegengesetzten Seiten ( $(25)$. Hier traten bei Beleuchtungen von 8 und 10 Minuten keine positive Krümmungen auf, wohl bei Beleuchtungen von 25 Minuten und länger. Zum Schlusz kann noch daran erinnert werden, dasz auch bei einseitigen Beleuchtungen bei Ueberschreitung einer gewissen Reizdauer keine negativen Krümmungen mehr auftreten. (vergl. § 14).

Neben den schon besprochenen Beobachtungen musz hier noch der Resultat einer Rotation bei 350 M.K. mitgeteilt werden. Bei Vorbeleuchtungen von 10, 36 und 100 Sekunden treten gar keine deutliche Krümmungen auf. Wird aber während 20 Minuten bei dieser Intensität rotiert, dann treten bei einer Nachbeleuchtung von 12000 M.K.S. positive Krümmungen auf, also auch hier dieselben Erscheinungen, welche schon ausführlich besprochen worden sind. Wenn man die hier erhaltenen Ergebnisse mit den Untersuchungen $\mathrm{Pr}$ ingsheims und Clarks vergleicht, dann ergibt es sich dasz es nicht möglich ist die hier erhaltenen Zahlen mit denen Pringsheims zu vergleichen. Pringsheim hat aber ganz richtig festgestellt, dasz bei vorbeleuchteten Pflanzen mit einer gröszeren Energiemenge gereizt werden musz um eine positive Krümmung $z \mathrm{u}$ bekommen als bei im Dunklen gezogenen.

Clarks Figur 2 gibt eine Uebersicht seiner Versuche, welche sich alle auf eine Intensität van $16 \mathrm{M}$.K. beziehen. Seine Beobachtungen stimmen sehr gut mit den hier mitgeteilten überein. Er hat auch die hier sub 2, 3 und 4 
besprochenen Erscheinungen beobachtet. Es ist aber aus den hier mitgeteilten Versuchen hervorgegangen, dasz seine Beschwerden gegen die von Pringsheim behauptete geringere Empfindlichkeit vorbeleuchteter Pflanzen ungegründet sind.

Bei Clarks Figur musz man natürlich immer daran denken, dasz er nicht vorhergesehen hat, dasz geotropische Gegenkrümmungen den Eindruck einer negativen Reaktion geben können. Auszerdem ist er gewohnt bei einer positiven Krümmung mit nachfolgender entgegengesetzt gerichteter nur diese negative Krümmung zu erwähnen. Es musz wahrscheinlich diesem Umstande zugeschrieben werden, dasz er die negative Reaktion über ein gröszeres Gebiet beobachtet hat. Seine Versuche sind in soweit abweichend, dasz er die Energie der einseitigen Nachbeleuchtung nicht in kurzer Zeit zuführt. Wenn er eine schwächere Intensität $z$. B. die der Tabelle 24 5,5 M.K. benutzt hätte würde das zu stärker abweichenden Resul taten geführt haben. Da hier aber keine Versuche mit einer Nachbeleuchtung, welche in langer Zeit zugeführt wird, ausgeführt worden sind, ist es nicht möglich zu sagen welche Unterschiede das verursacht hätte.

\section{$\S 31$. Eine allseitige Beleuchtung der eine} einseitige vorhergeht.

Pringsheim hat mitgeteilt, dasz wenn nach einer einseitigen Beleuchtung allseitig Licht zugeführt wird, negative Krümmungen hinsichtlich der einseitigen Vorbeleuchtung entstehen. Clark hat das in einer groszen Anzahl Versuche bestätigt. Es ist nicht nötig hier eine ausführliche Auseinandersetzung über die Ergebnisse dieser Beleuchtungsweise zu geben. Eine Reihe in der Tabelle 29 zusammen gebrachten Versuche macht es möglich diese Erscheinung zu vergleichen mit den zweiseitigen Beleuchtungen, welche 
Tabelle 29.

Eine einseitige beleuchtung der eine allseitige folgt.

Intensität der Vor - und Nachbeleuchtung.

+ bedeutet Krümmung nach der Lichtquelle der ersten einseitigen Beleuchtung.

- bedeutet Krümmung nach der entgegengesetzten Seite.

\begin{tabular}{|c|c|c|c|c|}
\hline \multirow{2}{*}{$\begin{array}{c}\text { Dauer der } \\
\text { einseitigen } \\
\text { Vorbeleuchtung. }\end{array}$} & \multicolumn{4}{|c|}{ Dauer der allseitigen Nachbeleuchtung. } \\
\hline & 35 Sek. & 100 Sek. & 300 Sek. & 600 Sek. \\
\hline 30 Sek. & ++ & \pm & 一 & $\overline{0}$ \\
\hline 60 Sek. & ++ & $\pm ?$ & $\pm ? ?$ & - \\
\hline 180 Sek. & + & Spitze- & \pm & 一 \\
\hline 300 Sek. & \pm & $\pm ?$ & $\pm ?$ & - \\
\hline
\end{tabular}

einer einseitigen folgen, welche schon in $\S 27$ besprochen worden sind. Ebenso wie dort (Tabelle 22 p. 132) zuerst eine Krümmung in Sinne der längerdauernden und später eine im Sinne der kürzerdauernden und auch später anfangenden Beleuchtung sichtbar wird, so tritt auch hier meistens zuerst eine Krümmung im Sinne der einseitigen Vorbeleuchtung auf und erst später eine in der entgegengesetzten Richtung. Bei all diesen Versuchen sind Kontrollbestimmungen auf dem Klinostaten verrichtet worden. Wegen der eklatanten Ulebereinstimmung mit den Resultaten einer zweiseitigen Nachbeleuchtung sind diese Versuche eine angenehme Befestigung dasz eine allseitige Beleuchtung als eine allseitige Reizung aufgefaszt werden musz wovon jeder Reiz gesondert von der Pflanze per- 
zipiert wird und eine Reaktion verursacht. Ebenso wie es in der Tabelle 22 nicht möglich war in allen Fällen zu bestimmen, wie eine Krümmung in entgegengesetzter Richtung hinsichtlich der einseitigen Beleuchtung aufgefaszt werden muszte, als positive hinsichtlich der kürzerdauernden Reizung oder als negative hinsichtlich der einseitig vorbeleuchteten Seite oder als Kombination beider, ist es auch hier nicht möglich diese Grenze zu ziehen. Mit vollkommener Sicherheit kann aber festgestellt werden, dasz auch bei Lichtmengen, wobei von einer negativen Reaktion nicht die Rede sein kann diese Erscheinungen auftreten. (vergl. \$33).

Clark hat seine Resultate in den Figuren 4 und 5 zusammengefaszt. Wenn man diese mit den hier in der Tabelle 28 gegebenen vergleicht und in Anmerkung nimmt dass Clark gewohnt ist

$1^{0}$. eine vorhergehende positive Krümmung nicht $z u$ erwähnen,

$2^{0}$. keine Kontrollversuche auf dem Klinostaten anzustellen,

$3^{0}$. jede Krümmung, welche der einseitigen Vorbeleuchtung entgegengesetzt gerichtet ist negativ zu nennen, dann ist die Ulebereinstimmung sehr befriedigend. 


\section{ABSCHNITT VII.}

\section{Das Abklingen.}

§32. Das Abklingen einer allseitigen Beleuchtung.

Die allseitige Vorbeleuchtung macht es möglich eine Erscheinung zu untersuchen, welche gewöhnlich das Abklingen einer Excitation genannt wird. Wenn man eine Pflanze während kürzerer oder längerer Zeit allseitig reizt, wird eine gewisse Unempfindlichkeit erreicht. Es ist nun möglich zu untersuchen, wie lange die Unempfindlichkeit bestehen bleibt und wie schnell sie erlischt. Dazu müssen die Pflanzen nach der Rotation eine gewisse Zeit im Dunkeln gelassen werden. Die geringere Empfindlichkeit, welche in diesen Augenblicken dann noch besteht, kann durch Feststellung der Reaktionsstärke auf einen Reiz von bestimmter Grösze bestimmt werden. Auf die theoretische Möglichkeit in dieser Weise das Abklingen einer Excitation zu bestimmen ist schon von $\mathrm{Pring}$ sheim hingewiesen worden.

Diese Versuche sind genau auf dieselbe Weise und mit derselben Methodik verrichtet als die, welche im vorigen Abschnitte besprochen worden sind. Gewählt ist die Untersuchung der Abklingungsweise einer Vorbeleuchtung mit einer Intensität von 25 M.K. während 100 Sekunden und während 20 Minuten.

Wie aus der Tabelle 26 hervorgeht ist nach einer Rotation van 100 Sekunden mit einer Intensität von 25 
M.K. keine positive Reaktion mehr zu bekommen. Es war interessant zu wissen ob das Reaktionsvermögen für positive Krümmungen wieder zurückkehren würde und wie das geschehen würde. Die Tabelle 39 enthält die hierfür nötigen Data. In der linken Kolumne ist die Zeit zwischen allseitiger Vor- und einseitiger Nachbeleuchtung angegeben worden. Die Energiemengen der einseitigen Nachbeleuchtung sind dieselben wie in den Tabellen 23 bis 27.

Zum Vergleich ist in der untersten horizontalen Kolumne die Reaktion auf die einseitige Beleuchtung, wenn die Pflanzen gar nicht vorbeleuchtet worden sind, aufgenommen worden. Die erste horizontale Kolumne ist aus der Tabelle 26 der dritten horizontalen Reihe übergenommen.

$$
\text { Tabelle } 30 .
$$

Abklingen einer allseitigen Vorbeleuchtung.

Während 100 Sek. vorbeleuchtet mit einer Intensität von 25 M.K.

\begin{tabular}{|c|c|c|c|c|c|c|c|c|c|}
\hline \multirow{2}{*}{$\begin{array}{l}\text { Zeit zwischen } \\
\text { Vor- und } \\
\text { Nachbeleuch- } \\
\text { tung. }\end{array}$} & \multicolumn{9}{|c|}{ Energie der einseitigen Nachbeleuchtung in M.K.S. } \\
\hline & 22 & 44 & 125 & 250 & 500 & 1000 & 4500 & 13.500 & 27.000 \\
\hline sogleich & & & & 0 & $?$ & ? & $-?$ & - & - \\
\hline 1 Min. & & & & & $+?$ & $\begin{array}{l}+? \\
0\end{array}$ & - & - & 一 \\
\hline 5 Min. & & & + & + & ++ & ++ & ++ & $\pm ?$ & - \\
\hline 20 Min. & & + & ++ & ++ & ++ & ++ & ++ & $\begin{array}{c}\text { +schwach } \\
0\end{array}$ & $-?$ \\
\hline 1 Stunde & + & ++ & ++ & ++ & ++ & ++ & + & 0 & $-?$ \\
\hline $\begin{array}{l}\text { Nicht } \\
\text { vorbeleuchtet }\end{array}$ & t- & ++ & ++ & ++ & ++ & ++ & \pm & - & - \\
\hline
\end{tabular}


Wenn nur eine Minute $z$ wischen dem Ende der allseitigen Vorbeleuchtung und dem Anfange der einseitigen Nachbeleuchtung verlaufen ist, ist es schon möglich eine schwache positive Reaktion zu bekommen. Allmählich kommt die Empfindlichkeit wieder zurück, sodasz nach einer Stunde fast das ursprüngliche Reaktionsvermögen wieder hergestellt ist.

Bei den starken Energiemengen, welche eine negative Krümmung verursachen, ist noch etwas Merkwürdiges zu bemerken. Bei einer einseitigen Nachbeleuchtung mit 4500 M.K.S. treten, wenn sogleich nachbeleuchtet wird, negative Krümmungen auf. Nach 5 Minuten Warten zwischen Vor- und Nachbeleuchtung werden nicht mehr diese sondern positive sichtbar. Auch bei Nachbeleuchtung mit 13500 M.K.S. und 27000 M.K.S. ist dies, wenn auch schwächer, zu beobachten. Um die Ursache dieser Erscheinung zu verstehen, musz man sich erinnern, das in der Tabelle 26 bei einer längeren Vorbeleuchtung als 3 Minuten, z. B. von 5 Minuten keine negative Krümmungen mehr erreicht werden. Es scheint erlaubt $z u$ sein zwischen diesen zwei Erscheinungen Zusammenhang zu suchen, sodasz man sich denken kann, dasz das nicht mehr Auftreten negativer Krümmungen bei dieser starken einseitigen Nachbeleuchtung die Folge eines Prozesses ist, welche abhängig ist von der Energiemenge womit vorbeleuchtet worden ist, welcher aber eine gewisse Zeit eingewirkt haben musz um diese Erscheinung zu verursachen (vergl. § 38).

Aus der Tabelle 30 geht hervor, dasz, wenn zwischen Vor- und Nachbeleuchtung eine Stunde verlaufen ist, die Möglichkeit negative Krümmungen $z u$ bekommen wieder einigermaszen hergestellt ist. Für die positive Krümmung ist die Pflanze nach dieser Zeit ganz in den ursprünglichen Zustand zurückgekommen aber wie aus dem Vergleich mit der untersten horizontalen Kolumne hervorgeht noch nicht ganz für die negative Reaktion. 
Tabelle 31 .

Abklingen einer allseitigen Vorbeleuchtung.

Während 20 Minuten allseitig vorbeleuchtet mit einer Intensität von $25 \mathrm{M} . \mathrm{K}$.

\begin{tabular}{|c|c|c|c|c|c|c|c|c|c|}
\hline \multirow{2}{*}{$\begin{array}{l}\text { Zeit zwischen } \\
\text { Vor- und } \\
\text { Nachbeleuch- } \\
\text { tung. }\end{array}$} & \multicolumn{9}{|c|}{ Energie der einseitigen Nachbeleuchtung in M.K.S, } \\
\hline & 22 & 44 & 125 & 250 & 500 & 1000 & 4500 & 13.500 & 27.000 \\
\hline sogleich & & & & 0 & + & ++ & ++ & ++ & ++ \\
\hline 1 Min. & & & & $+?$ & + & $+t$ & ++ & ++ & ++ \\
\hline 5 Min. & & & $+?$ & + & ++ & $+t$ & ++ & ++ & + \\
\hline 20 Min. & & $+?$ & ++ & ++ & ++ & ++ & ++ & + & $\pm ?$ \\
\hline 1 Stunde & $+?$ & + & ++ & ++ & ++ & ++ & $\begin{array}{c}++ \\
+\end{array}$ & $\frac{ \pm}{0} ?$ & $-?$ \\
\hline $\begin{array}{c}\text { Nicht } \\
\text { vorbeleuchtet }\end{array}$ & + & ++ & ++ & ++ & ++ & ++ & \pm & - & - \\
\hline
\end{tabular}

Die Tabelle 31 gibt das Abklingen einer allseitigen Vorbeleuchtung mit derselben Intensität (25 M.K.) welche 20 Minuten gedauert hat. Wenn sogleich einseitig nachbeleuchtet wird, ist eine negative Reaktion nicht $z u$ bekommen, sondern treten von 500 M.K.S. bis zu 27000 M.K.S. starke positive Krümmungen auf. Auch hier sieht man, wenn längere Zeit zwischen der allseitigen Vorbeleuchtung und der einseitigen Nachbeleuchtung verläuft, die Empfindlichkeit für positive Krümmungen zurückkehren; damit geht zusammen die Möglichkeit für das Auftreten einer negativen Reaktion bei starken Nachbeleuchtungen. Nach einer Stunde ist fast die ursprüngliche Empfindlichkeit wieder hergestellt; 22 M.K.S. geben eine schwache 
positive Krümmung und 27000 M.K.S. geben wieder eine negative Reaktion.

Wie die Abnahme der Empfindichkeit durch Vorbeleuchtung in der Tabelle 28 quantitativ festgelegt worden ist, sind auch über der Rückkehr der Empfindlichkeit einige quantitative Bestimmungen verrichtet worden. Diese Werte beziehen sich auf eine allseitige Vorbeleuchtung von 73 M.K. während 60 Sek. Bei einer einseitigen Nachbeleuchtung, welche unmittelbar folgt, ist keine positive Reaktion zu bekommen. Auch hier ist wie in der Tabelle 28 die Energiemenge bestimmt worden, womit einseitig nachbeleuchtet werden musz um nach $1 \frac{1}{2}$ Stunde eine Krümmung von $1 \mathrm{mM}$. zu bekommen.

$$
\text { Tabelle } 32 \text {. }
$$

Abklingen einer allseitigen Vorbeleuchtung mit einer Intensität von 73 M.K. während 60 Sek. eine positive Krümmung von $1 \mathrm{mM}$. gibt.

\begin{tabular}{r|r} 
folgt sogleich & keine Reaktion. \\
nach 12 Sek. & keine Reaktion. \\
30 Sek. & 1540 M.K.S. \\
2 Min. & 730 M.K.S. \\
8 Min. & 104 M.K.S. \\
30 Min. & 30 M.K.S. \\
60 Min. & 25 M.K.S.
\end{tabular}

Diese Tabelle stützt die schon mitgeteilten Beobachtungen.

\$33. Eine einseitige Beleuchtung der eine einseitige der entgegengesetzten Seite nicht sogleich folgt.

Nach Analogie des im vorigen Paragrafen besprochenen Abklingens einer allseitigen Vorbeleuchtung kann man die 
Frage stellen, welchen Einflusz es hat wenn zwischen zwei entgegengesetzt gerichteten gleich starken Beleuchtungen eine gewisse Zeit verläuft. Tabelle 33 zeigt die schon früher besprochene Erscheinung, dasz je nachdem zwischen zwei Beleuchtungen längere Zeit verläuft, die Möglichkeit zunimmt, dasz jede der Reizungen sich in einer Krümmung äuszert. Nur dann wird diese Erscheinung verständlich sein, wenn man annimmt dasz beide Krümmungsprozesse fast unabhängig von einander stattfinden.

$$
\text { Tabelle } 33 .
$$

105 M.K.S. (7 Sek.) sogleich danach die entgegengesetzte Seite 105 M.K.S. (7 Sek.)

Zeit zwischen den 2 Reizen 1 Min.

keine Krümmung

keine Krümmung

2 Min. erst Spitze 1 dann

Spitze 2

4 Min. erst 1 dann schwach 2

8 Min. erst 1 dann stark 2

§34. Eine einseitige Beleuchtung der eine allseitige nicht sogleich folgt.

Im Paragrafen 31 ist besprochen worden, dasz wenn einer einseitigen Beleuchtung eine allseitige folgt, meistens zuerst eine Krümmung im Sinne der einseitigen Beleuchtung sichtbar wird indem später eine entgegengesetzt gerichtete folgt. Aus der Tabelle 34 geht hervor, dasz wenn zwischen dem Zuführen der einseitigen und der allseitigen Beleuchtung eine gewisse Zeit verläuft, diese Erscheinung noch stärker hervortritt. Wird $z$. B. erst mit 125 M.K.S. einseitig beleuchtet und gleich danach 250 M.K.S. allseitig zugeführt, dann tritt allein eine Krümmung im Sinne der einseitigen Vorbeleuchtung auf. 
Tabelle 34 .

bedeutet gekrümmt nach der Lichtquelle der einseitigen Vorbeleuchtung.

- bedeutet gekrümmt nach der entgegengesetzten Seite.

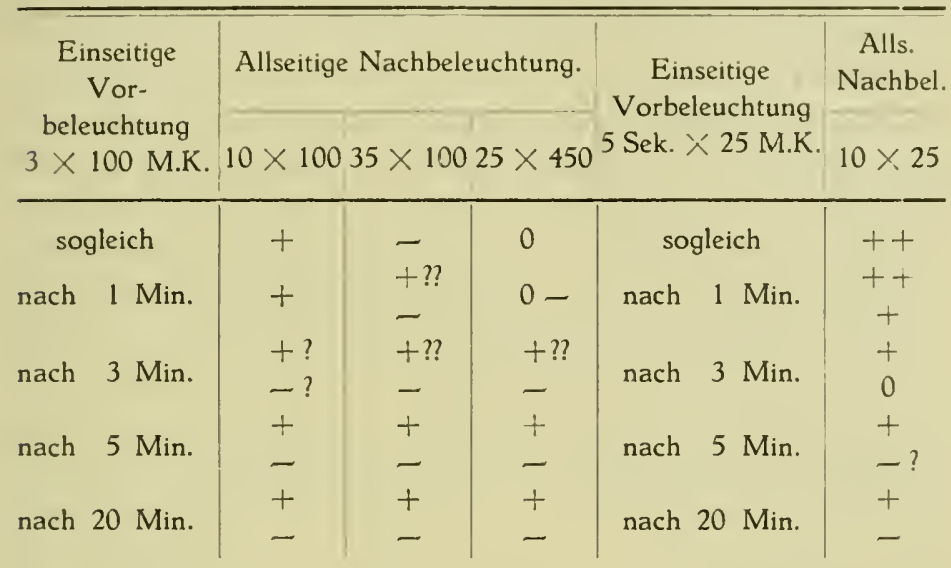

Je nachdem längere Zeit zwischen den zwei Beleuchtungen verläuft, nimmt die Möglichkeit zu dasz auch die entgegengesetzt gerichtete Krümmung sichtbar wird. So geht aus der Tabelle 34 hervor, dasz bei einer Ruhezeit von 20 Minuten die beiden Krümmungen geschieden auftreten. Bei diesen Energiemengen kann von einer negativen Reaktion nicht die Rede sein. Man kann hier also mit Sicherheit schlieszen, dasz die Krümmung, welche der einseitigen. Vorbeleuchtung entgegengesetzt gerichtet ist, als eine positive Krümmung betrachtet werden musz, welche durch die bei der allseitigen Beleuchtung an der nicht vorbeleuchteten Seite zugeführte Energiemenge verursacht wird. Auch hier zeigt es sich wieder ganz deutlich, dasz die allseitige Beleuchtung als eine Kombination einseitiger Reizreaktionen betrachtet werden musz. Die weiter noch in der Tabelle 34 mitgeteilten Werte zeigen für gröszere Energiemengen dieselbe Erscheinung. 


\section{$\S 35$. Zusammenfassung.}

Bei all den Versuchen, welche in diesem Abschnitte beschrieben worden sind, hat es sich gezeigt, dasz es einen groszen Unterschied macht ob zwei Beleuchtungen unmittelbar aufeinander folgen oder dasz eine gewisse Zeit dazwischen verläuft.

Wenn zwischen zwei Beleuchtungen von entgegengesetzten Seiten einige Zeit verstrichen ist, äuszern sie sich jede in einer Krümmung. ( $(33)$. Ist die zweite Beleuchtung eine allseitige, so wird doch eine Scheidung der zwei Beleuchtungen auftreten d. h. bei der allseitigen Beleuchtung heben die ebensostarken entgegengesetzt gerichteten Reize einander nicht auf. Je längere Zeit zwischen Vor - und Nachbeleuchtung verläuft, desto stärker äuszern sich die Krümmungen jede für sich. Das ist verständlich wenn man in Anmerkung nimmt dasz ein Reiz viel früher angefangen hat als der andere denn dann ist das nur eine Ausbreitung des Vermögens zwei entgegengesetzt gerichteter Krümmungen, sich jede stärker in einer Krümmung zu äuszern, je nachdem die Zeit, welche zwischen dem Anfange ihrer Induktion verläuft, gröszer ist.

Es ist nicht wahrscheinlich, dasz diese Erscheinung beruht auf einer Aenderung der Empfindlichkeit in der Seite, welche durch die einseitige Beleuchtung vorbeleuchtet worden ist. (vergl. § 37).

Wohl ist es möglich, dasz wenn lange Zeit zwischen einer einseitigen Vor - und einer allseitigen Nachbeleuchtung verläuft, die Krümmung schon eine Spitzenasymmetrie verursacht hat und diese eine Aenderung der Beleuchtungsumstände zu Folge hat. Bei der Studierung der von Clark mitgeteilten Erscheinung, dasz auch durch einseitige geotropische Reizung mit nachfolgender allseitigen Beleuchtung eine Reaktion sichtbar wird, welche dem geotropischen Reize entgegengesetzt gerichtet ist, wird man dieses in 
Betracht ziehen müssen. Auch die Versuche über das Abklingen einer allseitigen Vorbeleuchtung haben auf das, selbe Prinzip gewiesen, dasz zwei Krümmungen einander weniger entgegenwirken je nachdem längere Zeit dazwischen verlaufen ist. Hier wird die Summation mit der Energiemenge, welche an derselben Seite zugeführt worden ist bei der allseitigen Beleuchtung, ziemlich leicht stattfinden können wenn nur kurze Zeit seit der allseitigen Vorbeleuchtung verstrichen ist. In dem Masze wie die Zeit zwischen Vor- und Nachbeleuchtung länger ist, wird diese Summation geringer werden und zu gleicher Zeit die Gegenwirkung der Krümmung der anderen Seite weniger Einflusz haben. Da hier aber der Anfang aller Induktionen gleichzeitig ist, wird die Energiemenge, welche an der nicht nachbeleuchteten Seite zugeführt wurde, sich nicht in einer Krümmung äuszern, und nur der Krümmung der anderen Seite entgegenwirken können. 


\section{T E I L I I I. \\ Theoretische Betrachtungen.}

§36. Die Kompliziertheit der Reizprozesse.

Man ist gewohnt die Reizerscheinungen als Auslösungsvorgänge zu betrachten; da diese aber ebensogut bei nicht vitalen als bei vitalen Prozessen auftreten, hat man auf diese Weise die Reizreaktionen zwar einer umfangreichen Gruppe von Erscheinungen untergeordnet, sich aber einer befriedigenden Erklärung nicht genähert. Man ist tief durchdrungen von der auszerordentlichen Kompliziertheit der Reizprozesse. Dadurch ist man dazu gekommen zu versuchen die tropistischen Reizprozesse in einfachere $z u$ teilen. So hat man bei der photo- und geotropischen Reizreaktion drei Teilprozesse unterschieden. Es sind die perzeptorische, die duktorische und die Krümmungsphase. Es ist nicht $z u$ verneinen, dasz in vielen Fällen diese Unterscheidung berechtigt scheint. Das ist dann der Fall wenn die Krümmung in einem anderen Teile der Pflanze stattfindet als der Perzeptionsprozesz. Es ist aber erwünscht, darauf hinzuweisen, dasz wenn derselbe Teil der Pflanze, welcher den Reiz perzipiert auch die Krümmung ausführt, die logische Notwendigkeit einer dergleichen Einteilung fehlt. Man ist aber gewohnt in diesem Falle anzuführen dasz doch immer einige Zeit verläuft bevor die Krümmung auch des gereizten Teiles sichtbar wird und man stellt sich vor dasz in dieser sogenanten Latenzzeit die duktorischen Prozesse stattfänden. Die im expe- 
rimentellen Teil mitgeteilten Resultate weisen darauf hin, dasz man mit dergleichen aprioristischen Betrachtungen sehr vorsichtig sein soll. Zwar ist es nicht möglich das Bestehen einer duktorischen Phase zu leugnen, doch hat es sich gezeigt, dasz wenn sie besteht sie sicher kleiner sein musz als von den meisten Untersuchern bisher angenommen wurde.

Die Vorstellung, welche man sich bisher von der tropistischen Reaktion gemacht hat, beruht fast ganz auf Hypothesen. Man denkt sich eine Kette von Auslösungsprozessen wovon jeder Prozesz den folgenden auslöst. Selbst geht man so weit anzunehmen, dasz auszerdem jede Reizreaktion neben dem in der Kette folgenden noch eine zweite auslösen würde, welche bestrebt wäre den ursprünglichen Zustand wieder herzustellen. Dergleichen Vorstellungen haben den Nachteil, dasz sie die Beobachtungen und Versuche immer mehr oder weniger in eine bestimmte Richtung wenden. Es könnte darum besser scheinen in diesem Augenblicke eine abwartende Haltung anzunehmen, ins besondere da neue Untersuchungen immer mehr darauf hinweisen dasz die Kompliziertheit der Reizprozesse nicht so grosz ist als man sich vorgestellt hat. Die Möglichkeit wird man offen lassen müssen, dasz der tropistische Reizprozesz insoweit weniger kompliziert gedacht werden kann, dasz in dem Falle wo die Krümmung auf derselben Stelle stattfindet wie die Perzeption eine duktorische Phase fehlen kann.

Die mitgeteilten Versuche haben aber auf eine ganz andere Weise von Komplikationen hingewiesen. Neben einander können im Koleoptyle eines Avenakeimlinges mehrere Reaktionen verlaufen. Positive und negative Reaktionen nach allen Seiten gerichtet kommen in allen Teilen des Keimlinges in der Spitze wie in der Basis mehr oder weniger unabhängig von einander zustande. Dadurch wird die Analyse der phototropischen Reizprozesse auszer- 
ordentlich erschwert. Ob man dazu berechtigt ist die Reaktion einer einzigen Zelle als einheitlich zu betrachten und die des Keimlinges als Resultante der gesammten Zellreaktionen, oder dasz diese Vorstellung ebenso falsch ist als die dasz der Keimling einheitlich reagiert wird durch künftige Untersuchungen entschieden werden müssen.

\section{§37. Die Energiehypothese und die} Produktregel.

Fröschel ${ }^{1}$ ) und Blaauw ${ }^{2}$ ) haben gefunden, dasz bei Lichtreizung für den Schwellenwert der Reaktion das Produkt aus Lichtstärke und Beleuchtungsdauer konstant ist, d. h. dasz um eine eben merkbare Krümmung zu veranlassen es gleichgültig ist ob man längere oder kürzere Zeit mit schwacher oder mit starker Intensität reizt, wenn nur dafür gesorgt wird, dasz das Produkt von Lichtstärke und Reizdauer einen bestimmten Wert beträgt.

Frau Rutten-Pekelharing ${ }^{3}$ ) und Maillefer ${ }^{4}$ ) haben ein analoges Gesetz für Reizung mit Zentrifugalkraft festgestellt. Die einfache Beziehung, welche zwischen Kraft (Lichtstärke) und Reizdauer bestehen musz um einen minimum Effekt zu bekommen wird hier immer Produktregel genannt werden. Die Erklärung dieser Produktregel wird gefunden in der Hypothese, dasz die Stärke der Perzeption bestimmt

1) Fröschel, P. Untersuchung über die heliotropische Präsentationszeit. Sitz. ber. d. Kais. Akad. der Wiss. Wien Math. naturw. Kl. le Abt. Bd. CXVII 1908. Bd. CXVIII. 1909.

2) Blaauw, A. H. Die Perzeption des Lichtes. Recueil des Trav. Botan. Néerlandais. Vol. V. 1909.

3) Rutten-Pekelharing, C. J. Untersuchungen über die Perzeption des Schwerkraftreizes. Recueil des Trav. Botan. Néerl. Vol. VII. 1910.

4) Maillefer, A. Étude sur le géotropisme, Bull. d. 1. soc. vaud. 5me série Vol. XLV 1909. Vol. XLVI. 1910. 
wird durch das Produkt von Kraft (Lichtstärke) und Reizdauer. Beim Phototropismus hat dieses Produkt eine bestimmte Bedeutung. Es ist die zugeführte Energiemenge. Nimmt man an, das die Wirkung der Zentrifugalkraft kinetisch ist, dann wird hier eine gewisse Arbeit verrichtet. Es ist erwünscht diese Hypothese über die Art der Perzeption von der Produktregel unterscheiden zú können; darum wird sie hier als Energiehy pothese bezeichnet werden. Neben einander stehen also Produktregel und Energiehypothese; die Energiehypothese gibt eine Beziehung zwischen Reizstärke und Perzeption, die Produktregel zwischen Reizstärke und Reaktion. Die Wichtigkeit der Unterscheidung besteht darin, dasz die Gültigkeit der Energiehypothese unbeschränkt ist indem die Grenzen der Gültigkeit der Produktregel näher betrachtet werden müssen. Bei der Besprechung des Schwellenwertes ( $\$ 12)$ hat es sich gezeigt, dasz Blaauw nicht mit der wirklichen Schwelle gearbeitet hat. Mit Sicherheit konnte festgestellt werden, dasz schon nach Reizung mit 1 M.K.S. eine äuszerst schwache Reaktion sichtbar wird und nicht wie er meinte bei 20 M.K.S. die kleinste sichtbare Krümmung auftritt. Da also Blaauw mit einer Reaktion von bestimm ter meszbaren Stärke gearbeit hat, musz man sich fragen ob die Produktregel zufälligerweise für diese von ihm als Indikator gewählte Krümmungsstärke gültig ist oder dasz diese Regel für jede Krümmungsstärke gilt. In diesem Falle würde man eine grosze Ausbreitung der Produktregel bekommen und sie so definieren müssen um einen bestimmten Effekt zu bekommen ist es gleichgültig ob eine Energiemenge in kurzer oder langer Zeit zugeführt wird. Auf Grund von Versuchen mit groszen Energiemengen (§ 15) wobei negative Krümmungen auftreten, ist mit Sicherheit festzustellen, dasz das nicht der Fall ist, dasz die Produktregel nur eine beschränkte Gültigkeit hat.

Vor grosze Schwierigkeiten kommt man zu stehen, wenn 
man untersuchen will ob für die Energiemengen, welche starke positive Krümmungen verursachen die Produktregel gültig ist. Es versteht sich, dasz beim Vergleich von Krümmungen, durch Reizung mit einer selben Energiemenge während Zeiten welche sehr verschieden sind entstanden, die resultierenden Krümmungen als ungleichartige betrachtet werden müssen. (Vergl. $\S 9$ ). Wie schon früher auseinandergesetzt worden ist, besteht noch kein Mittel ungleichartige Krümmungen mit einander zu vergleichen. Man kann sich das am leichtesten verständlich machen, wenn man sich vorstellt welche Krümmungen man bekommt wenn 80 M.K.S. in 1 Sek. oder in 4 Stunden zugeführt werden. Die erste Krümmung wird schon nach 30 Minuten sichtbar und nach einer Stunde deutlich zu beobachten sein, die zweite musz viel später auftreten da nach 30 Minuten erst 10 M.K.S. nach einer Stunde nur 20 M.K.S. zugeführt worden sind und die dazu gehörigen Krümmungen eine gröszere experimentelle Reaktionszeit besitzen. An zweiter Stelle ist es experimentell nicht möglich einen Reiz während langer Zeit mit derselben Stärke zuzuführen. Die Ursache dessen ist, dasz die Pflanze zich während der Reizung krümmt. Aus den im Abschnitt IV mitgeteilten Resultaten von Versuchen, wobei das Licht unter verschiedenen Winkeln einfiel, ist hervorgegangen, dasz sobald die Spitze sich gekrümmt hat die Beleuchtung unter ganz abweichenden Verhältnissen stattfindet.

Die Frage ist ob die obengenannten Beschwerden nicht in Kraft waren bei den Untersuchungen van Blaauw und Fröschel. Es ist sehr wahrscheinlich dasz das wohl der Fall war. Mit Sicherheit ist festgestellt worden, dasz wenn mit einer Intensität van $1 / 1000$ M.K. beleuchtet wird, schon nach 170 Minuten $\left(16^{\circ} \mathrm{C}\right.$.) eine schwache Spitzenasymmetrie den Anfang der Krümmung andeutet. Um 20 M.K.S. mit dieser Intensität zuzuführen würde 
man 20000 Sekunden, das ist mehr als $5 \frac{1}{2}$ Stunde. reizen müssen. Während der ganzen Zeit, dasz die Spitze asymmetrisch ist sind die Beleuchtungsumstände geändert und kann sowohl Autotropismus wie geotropische Aufrichtung das Stärkerwerden der Krümmung entgegenarbeiten. Keiner der beiden Untersucher hat die Stärke der Krümmung beachtet, indem es auf Grund des obengesagten für unwahrscheinlich gehalten werden musz, dasz eine selbe Stärke bei langer und kurzer Dauer erreicht werden könnte. Es liegt also keine Veranlassung vor zu meinen, dasz die Gültigkeit der Produktregel selbst in diesem Falle streng bewiesen sei. Es wird aber äuszerst schwierig sein die Versuche so zu wiederholen, dasz es möglich ist Abweichungen von der Produktregel festzustellen. Jetzt kann nur zu einer ziemlich groszen Gültigkeit sowohl bei Schwerkraft als bei Lichtreizung geschlossen werden.

Die Produktregel hat eine merkwürdige Bestätigung gefunden bei der Untersuchung groszer Energiemengen. Im Abschnitte II $\S 15$ ist festgestellt worden, dasz auch für das Auftreten der negativen Reaktion die Produktregel gültig ist. Es ist sehr merkwürdig, dasz es gar keinen Unterschied macht ob solche grosze Energiemengen in wenigen Sekunden oder in vielen Minuten zugeführt werden. Es hat sich aber auch gezeigt, dasz eine negative Krummung nicht mehr auftritt wenn die Energiemenge nicht innerhalb einer bestimmten Zeit zugeführt wird, (etwa 25 Minuten). Nur bis zu diesem Wert von etwa 25 Minuten ist die Produktregel gültig. Es ist wohl einigermaszen begreiflich, dasz eine derartige Zeitgrenze für das Auftreten der negativen Krümmung bestehen musz, denn wenn eine grosze Energiemenge in langer Zeit zugeführt wird, musz die Aenderung, welche in der Pflanze beim Anfange entstanden ist, schon Veranlassung zu einer positiven Krümmung gegeben haben sodasz eine Zusammen- 
wirkung aller primairen Aenderungen zu einer negativen Reaktion nicht mehr stattfinden kann. Es ging aus den Versuchen hervor dasz diese Zeitgrenze etwa 25 Minuten beträgt. Nun tritt nach dieser selben Zeit auch die stärkere sichtbare Krümmungsreaktion auf. Das bringt uns dazu $z u$ denken dasz vielleicht eine gewisse Beziehung besteht zwischen dem nicht mehr Summieren der primairen Aenderungen und dem Auftreten der Krümmung. In diesem Falle würde das nicht mehr Zustandekommen der negativen Reaktion bei schwachen Intensitäten verursacht werden durch das stärkere Auftreten der Krümmung bevor die Energiemenge welche negative Krümmung veranlaszt zugeführt worden ist.

Das zweite Problem, welches gelöst werden musz, ist die Frage warum bei Beleuchtungen welche länger dauern als die welche negative Krümmung verursachen keine negative sondern nur positive Krümmungen auftreten. In $\S 17$ ist auseinandergesetzt worden dasz für das nicht mehr Auftreten der negativen Reaktion die Produktregel in viel geringerem Masze gilt. Mit Sicherheit konnte festgestellt werden dasz bei Intensitäten kleiner als 450 M.K. das Gebiet wo die negative Reaktion sichtbar ist immer kleiner wird. Bei gröszeren Intensitäten zeigten die Versuche auch, dasz das nicht mehr Auftreten der negativen Reaktion nicht bei einer konstanten Energiemenge stattfindet. Da aber diese Beobachtungen nicht ganz mit denen bei kleineren Intensitäten verglichen werden können (Abweichungen in Temperatur und Zusammensetzung des Lichtes) wird über die Möglichkeit dasz bei diesen das nicht mehr Auftreten der negativen Reaktion wohl von einer konstanten Energiemenge abhängig ist kein Urteil ausgesprochen werden.

$\mathrm{Clark}$ hat sich vorgestellt, dasz um diese sogenannte zweite positive Krümmung zu bekommen eine Erregungshöhe erreicht werden müsse, welche gröszer sei als die 
für die negative Reaktion. Nach seiner Auffassung würde also ein qualitativer Unterschied bestehen zwischen der ersten und der zweiten positiven Krümmung. In $\S 17$ sind die Gründe auseinandergesetzt worden warum diese Auffassung sehr unwahrscheinlich ist. Es zeigte sich, dasz die Beobachtungen vielmehr darauf hinweisen, dasz die negativen Erscheinungen auf die positiven superponiert sind und dasz ein Unterschied zwischen erster und zweiter positiver Krümmung nicht gemacht werden kann.

Pringsheim ${ }^{1}$ ) hat eine theoretische Vorstellung gegeben von den Prozessen, aie durch den Einflusz der Lichtreizung in der Pflanze stattfinden. $\mathrm{Da}$ seine Auffassungen sich im Laufe seiner Untersuchungen geändert haben ist es erwünscht darauf hinzuweisen dasz hier die Vorstellung besprochen wird welche er auf p. 459 u.f. der zweiten Mitteilung gegeben hat. Er denkt sich das Zustandekommen der negativen Reaktion abhängig von einer bestimmten Erregungsstärke. Nun tritt aber schon vom Anfange der Beleuchtung eine Aenderung der Lichtstimmung auf, welche verursacht dasz die Erregung abnimmt. „Es wird also nach einiger Zeit derselbe Reiz der im Beginne bei niedriger Siimmung, eine starke Erregung bewirkt hat an Wirkung verlieren, die Erregung wird sinken und kann sekundär unter das für negative Reaktion nötige Masz heruntergehen". Auf diese Weise versucht Pringsheim zu erklären, dasz bei einer starken Intensität bei kurzer Reizdauer negative, bei längerer positive Krümmung auftritt. Auch er nimmt also keinen prinzipiellen Unterschied zwischen erster und zweiter positiven Krïmmung an. Die Vorstellung ist sehr anziehend. Die grosze Beschwerde ist aber, dasz man sich in diesem Augen-

1) Pringsheim, E. Studien zur heliotropischen Stimmung und Präsentationszeit. Cohn's Beiträge zur Biologie der Pflanzen. Bd. IX. 1909. 
blicke noch gar keine Vorstellung davon machen kann wovon das Zustandekommen der negativen Krümmung abhängt und es noch nicht festgestellt is ob das nicht mehr Auftreten der negativen Reaktion bei stärkeren Intensitäten als 450 M.K.S. wirklich nicht bei einer konstanten Energiemenge stattfindet. Die Möglichkeit kann aber nicht geleugnet werden, dasz wie Pringsheim sich vorstellt eine Stimmungsänderung stattfindet; diese müszte dann aber bei stärkeren Intensitäten früher auftreten als bei schwächeren. (vergl. $\S 17$ ). Die Gültigkeit der Produktregel für die negative Reaktion ist nicht im Gegensatz zu Pringsheims Meinung dasz die Stimmungsänderung sogleich nach dem Beginne der Reizung anfange; nur ist es notwendig anzunehmen dasz der Betrag, womit die Erregung durch den Stimmungsprozesz abnimmt ebenfalls abhängig ist von der zugeführten Energiemenge.

In Zusammenhang mit der Frage wovon das Auftreten der negativen Reaktion abhängig ist, ist es erwünscht hier noch eine Erscheinung in Erinnerung zu bringen welche bei der Untersuchung zweiseitiger und allseitiger Beleuchtungen erhalten ist. In $\S 26$ hat es sich gezeigt, dasz wenn erst während einiger Zeit eine der Seiten eines Keimlings beleuchtet wird, bei einer Beleuchtung der entgegengesetzten Seite keine negative Krümmung erzielt werden kann. Auch bei einer zweiseitigen Beleuchtung mit folgender einseitiger $\S 27$ und bei einer allseitigen Beleuchtung mit folgender einseitiger ( $\$ 30$ sub 3 und 4) ist festgestellt worden, dasz die Möglichkeit negative Krümmungen $z u$ bekommen verschwinden kann. Wie dort ausführlich auseinandergesetzt worden ist, kommt die Pflanze bei kurzer zweiseitiger oder allseitiger Vorbeleuchtung in ein Stadium worin bei schwacher einseitiger Reizung positive, bei stärkerer negative Krümmungen erhalten werden. Hat die Vorbeleuchtung länger gedauert so kommt das Stadium worin nur negative Krümmungen 
$z u$ erzielen sind. Bei noch längerdauernden Vorbeleuchtungen treten aber nach allen einseitigen Reizen nur positive Krümmungen auf. Die Energiemenge wobei dann die positive Krümmung auftritt ist aber immer kleiner als die Energiemenge welche bei einer gewöhnlichen einseitigen Beleuchtung die „zweite positive Krümmung” veranlassen würde; auch wenn man bei der Energiemenge der einseitigen Nachbeleuchtung die schon bei der Vorbeleuchtung dieser Seite zugeführte Energiemenge in Anmerkung nimmt. Das musz dadurch verursacht werden dasz bei der Vorbeleuchtung der entgegengesetzten Seite Energie zugeführt worden ist. Ebenso wie das für die einseitige Beleuchtung auseinandergesetzt worden ist besteht auch bei den allseitigen Vorbeleuchtungen eine gewisse Beziehung zwischen Intensität und Reizdauer tür die zweite positive Krümmung. Auch hier tritt das Verlorengehen der Möglichkeit um negative Krümmungen zu erzielen schneller auf je nachdem die Intensität der Vorbeleuchtung stärker ist. Bei einer schwachen Intensität bleibt sie selbst so gering, dasz es immer möglich ist eine negative Krümmung durch starke einseitige Nachbeleuchtung zu bekommen. (vergl. Tab. $24-27$ ).

Die Untersuchung der Abklingprozesse ( $\$ 32$ ) hat gezeigt dasz dieselbe Erscheinung - das Verlorengehen der Möglichkeit um negative Krümmungen zu bekommen auch auftritt, wenn nach einer Vorbeleuchtung, welche zu dem Stadium geführt hat worauf eine einseitige Nachbeleuchtung keine positive Reaktion gibt und nur negative Krümmungen erhalten werden, eine Ruhepause kommt; es ist nicht unmöglich, dasz der Prozesz (der „Stimmungsprozesz") welcher das Verlorengehen der Möglichkeit um negative Krümmungen $z u$ erzielen verursacht hier nach dem Ende der Vorbeleuchtung weiter geht und zu Folge hat, dasz wenn einige Zeit später einseitig nachbeleuchtet wird wohl positive Krümmungen zu erzielen sind. Über 
die Art dieses sogenannten "Stimmungsprozesses" ist noch nichts zu sagen. Nur kann hier an eine interessante Beobachtung Pringsheims bei Keimlingen von Panicum erinnert werden, wobei die Perzeption und die Reaktion in verschiedenen Zonen lokalisiert sind. Hier zeigte sich dasz auch durch Vorbeleuchtung der Reaktionszone (Pflanzen mit umhüllter Spitze) eine Verkürzung der Reaktionszeit erhalten wurde. (p. 2881 Mitteil.) Pringsheim sagt „Das Ergebnis läst eine verschiedenartige Deutung zu. Scheinbar die einfachste ist die, dasz heliotropische Reizbarkeit und Umstimmungsfähigkeit verschieden lokalisiert sind und dasz letztere an die Krümmungsfähigkeit gebunden ist, die das Hypokotyl allein besitzt . . . ." Er fügt hinzu "Ebensogut kann eine Leitung des Stimmungsreizes in akropetaler Richtung nach der Koleoptyle stattfinden." Diese Beobachtung weist jedenfalls auf die Möglichkeit hin, dasz der sogenannte „Stimmungsprozesz” keine Empfindlichkeitsänderung verursacht, sondern in irgend einer Weise mit den späteren Phasen des Krümmungsprozesses zusammenhängt. $\mathrm{Ob}$ es wirklich erlaubt ist die hieroben besprochenen Erscheinungen mit einander in Zusammenhang $z \mathbf{u}$ bringen wird durch künftige Untersuchungen entschieden werden müssen.

Als Resultat der Besprechung der Gültigkeit der Produktregel für die negative Reaktion kann festgestellt werden, dasz nur bei Beleuchtungszeiten kleiner als 25 Minuten der negative Effekt bei derselben Energiemenge auftritt. Bei längeren Beleuchtungen ist die Regel nicht mehr gültig. Ueber das Ende der negativen Reaktion ist noch nichts mit Sicherheit zu sagen.

Es ist nun die Frage ob wenn die Produktregel nicht mehr gültig ist dennoch die Energiehypothese in Kraft sein kann. Bisher ist noch keine Veranlassung da anzunehmen dasz eine Grenze besteht für die Gültigkeit der Hypothese, dasz der primaire Prozesz, der in der Pflanze 
unter dem Einflusz des Lichtes stattfindet, bestimmt wird durch die zugeführte Energiemenge.

Neben dieser Fundamentalhypothese kann man noch eine zweite Voraussetzung machen, dasz nähmlich eine bestimmte Energiemenge immer eine gleichgrosze primaire Aenderung zustandebringt. Mit Pringsheim kann man sich denken dasz diese primaire Aenderung direkt proportional ist mit der zugeführten Energiemenge. Wenn sich also ergibt, dasz eine selbe Energiemenge in langer Zeit zugeführt nicht mehr denselben Effekt hat als in kurzer Zeit indem doch die primairen Aenderungen ebensogrosz sind, würde man schlieszen müssen, dasz das durch Prozesse ("Stimmungsprozesse") welche neben der Perzeption stattfinden verursacht wird.

Schon vor der Untersuchung Blaauws und Fröschels

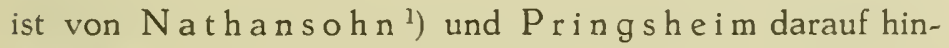
gewiesen worden, dasz die Stärke der Perzeptionsprozesse bestimmt wird durch die Energiemenge welche zugeführt wird. Sie waren dazu gekommen durch Resultate, welche sie bei intermittierender Lichtreizung erhalten hatten. Ihre Untersuchungen sind in diesem Augenblicke eine Stütze für die Energiehypothese ${ }^{2}$ ).

\section{$\S 38$. Das Abklingen.}

Es ist kein Zufall, dasz Nathansohn und Pringsheim bei ihrer Ưntersuchung mit intermittierenden Lichtreizen wohl die Energiehypothese gefunden haben, nicht aber zur Produktregel gekommen sind, die die Abhängigkeit des Effektes von der Reizstärke bestimmt. Mit groszer Wahr-

1) Nathansohn, A. und Pringsheim, E., Uber die Summation intermittierender Lichtreize. Jahrb. f. Wiss. Bot. Bd. 45. 1908.

2) Vergl. Hiley, W. E. On the Value of Different Degrees of Centrifugal Force as Geotropic Stimuli. Annals of Bot. Vol. XXVII, 1913. 
scheinlichkeit kann man die Ursache davon suchen in der Vorstellung über den tropistischen Reaktionsprozesz welche diese Untersucher mit vielen anderen teilen. Hier ist insbesondere die Vorstellung wichtig, dasz eine Erregung welche unter dem Einflusse eines-photo- oder geotropischen Reizes entstanden ist abklinge.

Pfeffer hat von einer Gegenreaktion gesprochen um anzugeben, dasz nach der Reizung die ursprüngliche Empfindlichkeit wieder erreicht wird. Dabei setzte er voraus, dasz bei einer Pflanze, welche schon gereizt worden ist, ein später kommender Reiz eine gröszere Stärke haben musz um eine Reaktion zu veranlassen als bei einer vorher nicht gereizten Pflanze. Die Rückkehr der ursprünglichen Empfindlichkeit kommt zustande durch das Abklingen der Erregung.

$\mathrm{N}$ athansohn und Pringsheim haben hierüber sehr interessante Betrachtungen geliefert. Das wichtigste ist im folgenden zusammengefaszt worden (1. c. p. 171). "Insgesamt haben wir uns also den heliotropischen Reizvorgang als eine Kette von Erregungserscheinungen zu denken von denen jede den Reiz für die folgende darstellt. . . . Damit ist aber die Reizkette noch nicht charakterisiert. Jedes einzelne ihrer Glieder löst nicht nur das folgende aus, das zur sichtbaren Reaktion, dem Ende der Kette führt, sondern noch ein zweites Glied die Gegenreaktion, welche das bestreben hat den auslösenden Erregungsvorgang zu beseitigen."

Von all diesen Prozessen ist nur das letzte Glied, der Krümmungsprozesz wahrnehmbar. Von diesem ist bekannt, dasz eine Krümmung wenn sie wenigstens nicht dadurch fixiert wird, dasz der gekrümmte Teil der Pflanze nicht mehr wächst, nach einiger Zeit zurückgeht. (Autotropismus, Rektipetalität).

Indirekt kann hieraus abgeleitet werden, dasz die bei der Perzeption induzierte Aenderung in diesem Augenblick 
nicht mehr besteht, dasz also ein Abklingen stattgefunden haben musz.

Die Abklingprozesse, welche in dieser Untersuchung studiert worden sind, beruhten alle auf demselben Prinzip. dasz zwei Krümmungen einander welliger entgegenarbeiten je nachdem längere Zeit zwischen dem Anfang ihrer Induktionen verlaufen ist. Das ist verständlich wenn man in Anmerkung nimmt, dasz das Augenblick worauf die Induktion anfängt auch den Augenblick bestimmt worauf die Reaktion beginnt. Man hat hier also nicht den Prozesz den man Abklingen einer Erregung zu nennen gewohnt ist. Dabei müszte durch einen vorhergehenden Reiz eine Abnahme der Empfindlichkeit auftreten, sodasz ein später hinzukommender Reiz eine schwächere primaire Aenderung veranlassen würde. Diese geringere Empfindlichkeit könnte dann wieder verschwinden und dadurch der ursprüngliche Zustand wieder hergestellt werden. Über die Vorkommen eines dergleichen Prozesses sind bisjetzt noch keine Anweisungen gefunden worden. Bei Versuchen wobei während langer Zeit mit groszer Lichtstärke vorbeleuchtet wurde z. B. bei einer allseitigen Vorbeleuchtung von 4 Stunden mit einer Lichtstärke von $30 \mathrm{M}$.K. trat noch gar keine Abnahme der Möglichkeit um positive Krümmungen zu erzielen auf. Eine Ermüdung kommt nicht vor.

Beim Phototropismus sind überhaupt keine Beobachtungen bekannt, welche etwas lehren über das Abklingen der primairen Aenderung, die unter dem Einflusse der Reizung in der Pflanze entsteht. Darum is es erwünscht einige Untersuchungen, welche hierüber beim Geotropismus Material geliefert haben näher $z u$ betrachten.

Zielinski ${ }^{1}$ ) hat angegeben wie er sich den Zusammenhang zwischen Erregung und Krümmungsstärke denkt.

1, Zielinski, T. Über die gegenseitige Abhängigkeit geotropischer Reizmomente. Zeitschr. f. Bot. Bd. 3. 1911. 
Er stellt sich vor dasz ein geotropischer Reiz von der Dauer der Präsentationszeit eine Erregung veranlaszt, die in dem Augenblicke, wo die Reaktion anfängt d. h. also am Ende der Reaktionszeit sich noch auf einer gewisser Höhe befinden musz um eine Krümmungsreaktion auszulösen.

Die Erregung, welche am Ende der Präsentationszeit besteht, würde durch Abklingprozesse während der Reaktionszeit abnehmen.

Dasselbe Prinzip findet man in den Vorstellung Fittings ${ }^{1}$ ) und vieler anderen zurück. Die Krümmungsstärke würde also abhängig sein von der Erregung, die am Ende der Reaktionszeit besteht und diese würde durch den Einflusz der Abklingprozesse immer geringer sein als die ursprünglich induzierte. Eine grosze Schwierigkeit für diese Auffassung liegt in der Gültigkeit der Produktregel. Noch gröszer ist diese wenn man annimmt, dasz auch während der Beleuchtung ein Abklingen stattfindet.

Fitting hat bei der Erklärung seiner Resultate über die Gültigkeit der Sinusregel bei dem Geotropismus eben dieses Abklingen benutzt um verständlich $z u$ machen, dasz ein Reiz von längerer Dauer stärker wirkt als einer von kürzerer Dauer auch wenn das Produkt $i \times t$ konstant ist. Er fand bei intermittierender Reizung in Winkeln von $90^{\circ}$ und $15^{\circ}$, wobei die Reizdauer in diesen Lagen respektivisch 2 und 10 Minuten betragen muszte damit die Pflanzen sich nicht krümmten (Relaxationsindex 1:5), dasz die Sinusregel ein anderes Verhältnis der Reizzeiten erforderte. Er schrieb diese Abweichung dem Abklingen des kurzdauernden Reizes in der Lage von 90 , während der viel längeren Dauer der anderen Reizung zu. Er setzte dabei voraus dasz das Abklingen nur während der Ruhezeit stattfinde. Dieses Ergebnis ist erstens nicht in Ueberein-

1) Fitting, H. Untersuchungen über den geotropischen Reizvorgang. Jahrb. f. Wiss. Bot. Bd. 41. 1905. 
stimmung mit Fittings eigenen Versuchen, der ebenfalls bei Vicia faba bei intermittierender Zuführung eines Reizes von der Totaldauer der Präsentationszeit bei dem selben Verhältnis von Reiz zu Ruhezeit wie oben (1:5) keine Verlängerung der Präsentationszeit bekam obwohl diese hätte resultieren müssen wenn wirklich in der Ruheperiode ein Abklingen stattgefunden hätte. Zweitens ist es hypothetisch, dasz das Abklingen nur während der Ruheperiode stattfindet, wenn nicht gereizt wird. Darin weicht Fitting $\mathrm{ab}$ von $\mathrm{N}$ ath ansohn und Pringsheim, die auf Grund von Resultaten welche sie bei intermittierender Lichtreizung erhalten hatten dazu schlieszen dasz das Abklingen während der Reizung und während der Ruheperiode auf dieselbe Weise stattfindet. Die $\mathrm{Ab}$ weich ung welche Fitting von der Sinusregel fand läszt sich also nicht durch Abklingen erklären und musz wahrscheinlich anderen Ursachen zugeschrieben werden (vergl. $\S 45$ und Fräul. Risz ${ }^{1}$ ).)

Fittings Untersuchung ist eigentlich die einzige. worin die Abklingsprozesse systematisch untersucht worden sind. Das von $\mathrm{OhnO}^{2}$ ) untersuchte Abklingen der Reaktionsprozeșe unter abnormen Umständen kann auszer Betracht gelassen werden, da aus dieser Untersuchung keine Folgerungen $\mathrm{zu}$ ziehen sind über das Abklingen unter normalen Umständen.

Fitting hat mit Hilfe eines intermittierenden Klinostaten die Relaxationszeit für geotropische Reizung bestimmt. Er definiert diese als „die Zeitdauer, die nötig ist bis die durch eine Reizung von kürzerer Dauer als die Präsentationszeit ausgelósten und auf die Reaktion hinzielenden

1) Risz, M. M. Über den Einfluss allseitig und in der Längsrichtung wirkender Schwerkraft auf Wurzeln. Jahrb. f. Wiss. Bot. 1913.

") Ohno, N. Über das Abklingen von geotropischen und heliotropischen Reizvorgängen. Bd. 45. 1908. 
Vorgänge nach Beseitigung des Reizanlasses gerade so weit ausklingen, dasz bei intermittierender Wiederholung gleicher Reizungen eine geotropische Krümmung nicht mehr eintritt." Er fand, dasz das Verhältnis von Reizdauer zu Relaxationszeit von der absoluten Dauer dieser Zeiten unabhängig ist.

Zielinski hat darum vorgestellt dieses Verhältnis von Reizdauer zu Relaxationszeit Relaxationsindex zu nennen. Bei der Besprechung von Fit $\mathrm{t}$ ing s Untersuchungen musz daran gedacht werden dasz die Produktregel damals noch unbekannt war. Da Fitting die Relaxationszeiten nur in der für die Schwerkraft optimalen Reizlage untersucht hat ist die Intensität der Kraft bei seinen Versuchen konstant. Dadurch ist die Präsentationszeit die Variable, wovon die Stärke des Krümmungsprozesses abhängig ist. Es wäre also besser unter $\mathrm{Relaxationsindex} \mathrm{nicht}$ mit Zielinski zu verstehen das Verhältnis von Reizdauer und Relaxationszeit da in diesem Falle dieser Name nur Bedeutung haben würde bei Reizung mit Schwerkraft in der optimalen Reizlage, sondern das Verhält nis von dem Produkt von Intensität und Reizdauer zu der Relaxationszeit. Bei Lichtreizung ist das also das Verhältnis der zugeführten Energiemenge zu der Relaxationszeit. Zi elins ki hat darauf hingewiesen, dasz der Relaxationsindex gröszer gefunden wird wenn die Pflanzen sich in der Ruhezeit nicht in der vertikalen Lage befinden sondern wenn sie um die horizontale Achse eines Klinostaten rotieren. Fräul. Risz hat das bestätigt; sie hat gezeigt, dasz die Kraft welche in der vertikalen Ruhelage in der Längsrichtung wirkt eine hemmende Wirkung auf die einseitige tropistische Reizung ausübt. $\mathrm{Fitting}$ hat gefunden dasz bei einer intermittierenden Reizung (Verhältnis $1: 6$ ) wobei die optimale Reizlage und die vertikale Ruhelage $\left(90^{\circ}\right.$ und $\left.0^{\circ}\right)$ kombiniert werden die Totaldauer der Reizzeiten unter diesen Umständen 
länger sein musz als die Präsentationszeit bei ununterbrochener Reizung beträgt. Daraus darf man nun nicht zu einem ziemlich schnellen Abklingen schlieszen, da ein ganz anderer Resultat erhalten sein würde, wenn nicht in den Ruhezeiten die Kraft, die in der Längsrichtung wirkt dem Zustandekommen einer Krümmung entgegengearbeitet hätte.

Es ist also nicht gestattet aus den Versuchen Fittings zu einem schnellen Abklingen das mit der Gültigkeit der Produktregel nicht $z u$ vereinigen wäre $z u$ schlieszen. Vielmehr geht es auch aus Beobachtungen Fittings selbst hervor, dasz sehr kleine Unregelmäszigkeiten bei der Drehung auf dem Klinostaten sich summieren können. Van $\mathrm{Harreveld}{ }^{\text {)}}$ ) fand, dasz selbst äuszerst winzige Reizungen allmählig $z u$ einer Krümmung Veranlassung geben. Darum ist es auch schwierig zu entscheiden ob die von $Z_{i}$ elinski angegebenen Zahlen für den Relaxationsindex Wert haben. Es läszt sich denken, dasz wenn die Versuchen nur genügend lange fortgesetzt werden ein noch gröszerer Index gefunden werden könnte.

$\mathrm{Maillefer}{ }^{2}$ ) hat zu zeigen versucht dasz jede geotropische Induktion eine Krümmung zu Folge hat. Wenn das wirklich richtig wäre, würde daraus folgen dasz man bei der Untersuchung dieser Relaxationserscheinungen eigentlich untersucht, wie lange Zeit zwischen zwei Induktionen verlaufen musz damit die Krümmungen sich noch zu einem makroskopisch wahrnehmbaren Effekt summieren können.

Pringsheim hat auch den Widerstreit gesehen, der gelegen ist in der sehr groszen Gültigkeit der Produktregel

1) Van Harreveld, Ph. Die Unzulänglichkeit der heutigen Klinostaten für reizphysiologische Untersuchungen. Recueil des trav. Bot. Neérlandais Vol. III. 1906.

$\Rightarrow$ Maillefer, A. Nouvelle étude experimentale sur le géotropisme. Bull. Soc. Vaudoise Vol. XLVIII, 1912. 
und dem Abklingen das den Effekt vermindern musz. Er findet die wahrscheinlichtste Erklärung der Produktregel „dasz der physiologische Effekt proportional mit jedem der zwei Faktoren (Zeit und Intensität) anwächst. Wenn nun die Erregung bis zu einer bestimmten Grenze proportional mit der Zeit zunimmt dann musz bei einem Reiz von der Dauer der Präsentationszeit die Gegenreaktion nicht in Anmerkung kommen. Die Erregung kann aber nicht andauernd geradlinig steigen, weil sonst ein Reiz von noch so geringer Intensität schlieszlich die maximale Reaktion hervorrufen müszte, was er erfahrungsgemäsz nicht tut. (2. Mitteil p. 456) Vielmehr entspricht jedem auch dem schwächsten Reizanlasse eine gewisse nicht überschreitbare und daher früher oder später konstant werdende Erregungshöhe, sodasz wir also nicht um die Forderung herum können, dasz die Gegenreaktion sofort erwachen müsse." Aus diesem Zitat ersieht man, dasz Pringsheim meint dasz nur wenn das Abklingen sehr langsam stattfinde die Produktregel zu verstehen sei. Aus seiner Auseinandersetzung geht hervor, dasz er ein Abklingen insbesondere darum annimmt da zu einer bestimmten Intensität eine bestimmte Erregungshöhe gehört. Es ist aber nicht ganz deutlich auf welchen Beobachtungen diese Auffassung sich gründet. Auch in der Untersuchung $\mathrm{Nath}$ a n sohns und $\mathrm{Pr}$ ing shei m s wird angenommen, dasz zu jeder Intensität eine bestimmte Erregungshöhe gehört (1. c. p. 171). Hier wird als Beweis angeführt, dasz Pflanzen welche von der Seite beleuchtet werden sich in einen bestimmten Winkel mit der Richtung des Lichtes stellen. Dieser Grenzwinkel würde durch die kombinierte Wirkung der Schwerkraft und des Lichtes bestimmt werden. Solange aber nicht bewiesen ist, dasz bei Reizung mit der Schwerkraft die Erregung eine konstante Höhe erreicht, scheint es nicht gestattet $\mathrm{N}$ athansohn und Pringsheim zu folgen und aus dem Konstantbleiben 
des Abweichungswinkels bei der Zusammenwirkung von Schwerkraft und Licht etwas abzuleiten über das Erreichen einer konstanten phototropischen Erregungshöhe. Man könnte sich vielleicht auch vorstellen dasz die Stärke welche die Krümmung erreicht wenn die Schwerkraft ihr nicht entgegenarbeitet einen Beweis für das Erreichen einer konstanten Erregungshöhe liefern könnte. Es zeigt sich aber, dasz auch dies nicht der Fall ist. Bei Schwerkraftreizung hat schon Fr. Darwin') gefunden dasz die Krümmung dauernd an Stärke zunimmt. Er fixierte die Spitze eines Keimlinges von Setaria in horizontaler Lage so dasz dieser empfindliche Teil fortdauernd gereizt wurde und eine immer gröszer werdende Krümmung der basalen Zonen verursachte. Auch bei dem Phototropismus zeigt es sich möglich, dasz mit der Dauer der Reizung die Krümmung fortwährend an Stärke zunimmt. Man findet hierüber einzelne Data in Teil I $\S 17$ indem die Fig. 4 auf Tafel I einen Avenakeimling vorstellt der 45 Minuten gereizt worden ist und sich auf dem Klinostaten so stark gekrümmt hat dasz er fast einen Kreis formt. Aus diesen Versuchen kann über die Stärke der Erregung nichts abgeleitet werden. Ein Beweis für oder gegen die Hypothese dasz die Erregung für jede Intensität eine konstante Höhe erreicht, ist also $\mathrm{nicht} z \mathrm{u}$ finden. Damit entsinkt der Forderung Pringsheims der Grund dasz ein Abklingen stattfinden müsze.

Aus den obigen Betrachtungen ergibt sich, dasz bisher keine Tatsachen bekannt sind über das Abklingen der Erregung die unter dem Einflusse eines geo- oder phototropischen Reizes entstanden ist. Mit Sicherheit ist von der Aenderung, welche in der Pflanzezu Folge der Reizung stattgefunden hat nur $z$ u sagen, dasz sie nicht unverändert bestehen

1) Darwin, Fr. Annals of Bot. 13. 1899. 
bleibt. Das geht z. B. hieraus hervor, dasz bei einem Reize der länger als 25 Minuten dauert keine Summation der primairen Aenderungen zu einer negativen Krümmung mehr stattfinden kann. Wie schon besprochen ist wird das wahrscheinlich dadurch verursacht, dasz die Krümmung nach etwa derselben Zeit stärker auftritt. In diesem Falle verschwindet wohl die primaire Aenderung doch nicht ohne einen Effekt verursacht zu haben. Dieses Verschwinden der Excitation ist denn auch nicht im Streit mit der Möglichkeit, dasz äuszerst schwache Reize sich nach einiger Zeit in einer Krümmung äuszern können. Es is t fraglich ob es wirklich ein Abklingen in dem Sinne gibt dasz die primaire Excitation verschwindet ohne $z u$ dem Effekt mitgewirkt $z u$ haben. (Siehe die Zusammenfassung).

\section{$\S 39$. Webersches Gesetz.}

Man hat sich vielfach die Frage gestellt ob auch bei Pflanzen eine ähnliche Beziehung zwischen Reiz und Reaktion zu finden sei als beim Menschen zwischen Reiz und Empfinding. Von mehreren Seiten ist dagegen Einwand erhoben worden, dasz man auf diese Weise die Gültigkeit eines psychischen Gesetzes zu finden versuchte damit man auch bei den Pflanzen von einer Empfindung reden könnte Dagegen ist darauf hingewiesen worden dasz das Webersche Gesetz bei den Pflanzen eine rein physiologische Bedeutung haben kann. Massa $\mathrm{t}^{\mathrm{l}}$ ) hat versucht es bei phototropischer Reizung von Sporangienträger von Phycomyces zurück zu finden. Nathansohn, Pringsheim und $\mathrm{Bla}$ a uw haben bezweifelt ob seine Folgerungen gestattet sind.

1) Massart, J. Recherches sur les organismes inférieures. La loi de Weber verifiée pour l'héliotropisme du champignon.

Bull. de l'Acad. roy. de Belgique. 1888 (3 sér. Bd. 16.) 
Auf Grund der Versuche welche in $\S 25$ über gleichzeitige Beleuchtung von zwei entgegengesetzten Seiten mitgeteilt worden sind, kann festgestellt werden dasz eine Pflanze zwei gleichzeitige Reize von entgegengesetzten Seiten jeden für sich perzipiert. Die Vorstellung, dasz ein Avenakeimling nur den Unterschied oder das Verhältnis der Reize empfinden würde ist ungegründet. Wenn man hier einmal die Beziehung welche das Webersche Gesetz fordert finden sollte dann könnte man mit Sicherheit sagen, dasz die Beziehung hier durch die einander entgegenarbeitenden Krümmungsprozesse bestimmt wird. Die hier mitgeteilten Data liefern kein genügendes Material über die Gültigkeit des Weberschen Gesetzes bei diesen $z$ weiseitigen Beleuchtungen ein Urteil auszusprechen.

\section{$\S 40$. Stim mung.}

Es ist erwünscht hier kurz zusammenzufassen, was diese Untersuchungen über das Stimmungsproblem Neues gebracht haben. Man hat sich gewöhnt von der Lichtstimmung einer Pflanze zu reden, wenn die Weise worauf eine Pflanze auf einen Reiz antwortet durch den Einflusz den ein vorhergehender Reiz ausgeübt hat geändert wird. Im experimentellen Teile ist so viel wie möglich vermieden worden das Wort Stimmung zu benutzen, da die Unbestimmtheit dieses Ausdrucks leicht zu Verwirrung Veranlassung geben kann. Pfeffer hat sie ganz allgemein definiert als die jeweilige Receptions- und Actionsfähigkeit.

Es hat sich nun gezeigt, dasz die Reaktion eines Avenakoleoptyls als die Resultante einer groszen Anzahl selbständig perzipierender und mehr oder weniger unabhängig von einander reagierender Teile aufgefaszt werden musz. $\mathrm{Da}$ nun Perzeption und Reaktion dieser Teile jede für sich durch verschiedene Umstände beeinfluszt werden kann und auch die Teilreaktionen einander mehr oder weniger 
entgegenwirken können, hat man zwei Erscheinungen von einander zu scheiden.

Eine zeigt sich ins besondere bei mehrseitigen Beleuchtungen und beruht auf der Zusammenwirkung verschiedener Reaktionsprozesse, wobei die entgegengesetzt gerichteten Krümmungsneigungen das Zustandekommen von Krümmungen zu verhindern versuchen. Wenn man in diesem Falle von einer Stimmungsänderung einer vorbeleuchteten Pflanze spricht, so musz man sich Rechenschaft geben dasz unter Stimmung nicht verstanden wird eine Empfindlichkeitszustand der in dem ganzen Organ auf dieselbe Weise anwesend zu sein braucht. Darum ist es auch nicht erwünscht in diesem Falle von einer hoch oder niedrig gestimmten Pflanze zu reden.

Pringsheim versteht unter Stimmung "den inneren Zustand, der die Lage der Cardinalpunkte bei einer Pflanzenart bestimmt": Cardinalpunkte sind nach ihm die Schwelle, das Optimum, die Indifferenzzone und die negative Schwelle. Einen derartigen Stimmungsprozesz die sich also auf die Perzeption oder Reaktion jeder Teilreaktion bezieht meint Pringsheim in der Erscheinung gefunden zu haben, dasz nach fortgesetzter Beleuchtung nicht mehr eine negative sondern eine sogenannte zweite positive Krümmung auftritt. In $\S 37$ ist eine ausführliche Auseinandersetzung der Data zu finden welche diese Untersuchung sowohl bei einseitigen als bei mehrseitigen Beleuchtungen geliefert hat. Die Erklärung, die Bla a uw für diese Erscheinung gegeben hat, ist mit Recht von Pringsheim (3. Mitteilung) bestritten worden. Es ist aber in diesem Augenblicke nicht möglich diese Erscheinung genauer zu analysieren. Schon in $\S 37$ ist darauf hingewiesen worden, dasz es in Bezug auf diesen Stimmungsprozesz noch unbekannt ist ob er eine Verminderung des Perzeptionsvermögens oder eine Aenderung in den späteren Stadien des Reaktionsprozesses verursacht. 
$\S 41$. Die negative Krümmung.

Von zahlreichen Untersuchern sind negative Krümmungen konstatiert worden. N. J. C. M ülle ${ }^{1}$ ) hat zuerst die Hypothese ausgesprochen, dasz bei jeder Pflanze positive und negative Krümmungen zu erzielen seien. Das hat sich immer mehr bestätigt. Oltmanns ${ }^{2}$ ), Figdor ${ }^{3}$ ), $\mathrm{Blaauw}, \mathrm{Vouk}{ }^{4}$ ) und $\mathrm{Clark}$ zeigten das für verschiedene Pflanzen. Aus den hier mitgeteilten Versuchen mit zweiseitiger Beleuchtung kann man ableiten, dasz eine negative Krümmung etwas ganz anderes ist als eine positive, denn es ist nicht möglich dasz eine negative Krümmung sich einfach mit einer positiven der entgegengesetzten Seite $z u$ einem stärkeren Effekt summiert. Merkwürdig ist die in $\S 13$ beschriebene Erscheinung, dasz in Folge einer einseitigen Beleuchtung von bestimmter Stärke eine negative Krümmung nach einer positiven auftritt. Es wäre nämlich bemerkenswert, dasz eine positive und eine negative Reaktion durch einselbes Organ zu gleicher Zeit perzipiert wird. Wenn auch diese Möglichkeit nicht auszuschlieszen ist, so kann dennoch in Erinnerung gebracht werden, dasz man sich davon auch eine andere Vorstellung machen kann.

Es läszt sich denken, dasz die Pflanze nicht überall gleich stark gereizt wird, da einige Teile weniger empfindlich sind, oder unter ungünstigen Verhältnissen beleuchtet werden. So werden die an der Seite gelegenen Teile und der schräg nach oben zulaufende Teil der Spitze

$\left.{ }^{1}\right)$ Müller, N. J. C. Botan. Unters. Leipzig. 1877.

2) Oltmanns, F. Uleber positiven und negativen Heliotropismus. Flora Vol. 83. 1897.

3) Figdor, W. Experimentelle Studien über die heliotropische Empfindlichkeit der Pflanzen. Wiesner Festschrift. 1908.

${ }^{4}$ ) Vouk, V. Zur Kenntnis des Phototropismus der Wurzeln. Sitz. ber. Ak. Wien M. N. Kl. Bd. 121. 1912. 
unter einem kleineren Winkel als $90^{\circ}$ gereizt werden. Nach dem Sinusgesetze werden sie weniger Licht empfangen als die Teile, welche unter einem Winkel von $90^{\circ}$ getroffen werden. Von diesen Teilen werden die stärkst beleuchteten eine negative, die schwächer gereizten eine positive Reaktion veranlassen können. Durch ihre $\mathrm{Zu}$ sammenwirkung kommt eine positive Krümmung zustande, welcher eine negative entgegenarbeitet, die sie zum Schlusz überwindet. Da es sich gezeigt hat dasz ein Koleoptyl nicht einheitlich den Reiz perzipiert, kann gegen diese letzte Vorstellung kein prinzipieller Einwand erhoben werden. Vielleicht wird es durch Beleuchtung eines kleinen Zellcomplexes oder eines einzelligen Organes möglich sein zu entscheiden ob wenigstens die Reaktion einer einzigen Zelle einheitlich ist oder ob auch hier eine positive und eine negative Reaktion zu gleicher Zeit entstehen können, wovon jede sich in einer Krümmung äuszert.

\section{$\S$ 42. Die Reizschwelle.}

Bei der Untersuchung reizphysiologischer Reaktionen hat man sich gewöhnt Schwellenbestimmungen auszuführen. Wenn die Reaktion, wie bei den hier untersuchten phototropischen Prozessen von der Energiemenge abhängig ist, dann könnte man einen Unterschied machen zwischen einer Schwelle für die Energiemenge, einer für die Lichtintensität und einer für die Reizzeit. Es musz aber erst untersucht werden ob Veranlassung vorliegt diese Schwellenwerte einzuführen.

Fitting hat die Zeitschwelle untersucht. Er versuchte für einen geotropischen Reiz die Perzeptionszeit festzustellen. Diese definierte er als ,diejenige minimale Zeitdauer, die dazu erforderlich ist, damit die Pflanze einen Reizanlasz perzipiert." Es ist ihm nicht gelungen diese Zeitschwelle bei intermittierender Reizung zu bestimmen. Nathansohn, 
Pringsheim und $\mathrm{Bla}$ a $\mathrm{u}$ finden das Bestehen einer Zeitschwelle fraglich. B la a u w äuszert sich sehr entschieden „einen absolute Zeit oder Intensitätsschwelle ist nicht ze erwarten". Experimentell hat Fröschel festgestellt, dasz bei einer Reizdauer von 1/2000 Sekunde ein phototropischer Reiz perzipiert wird, und es ist nicht einzusehen warum diese Zeit nicht willkürlich abgekürzt werden könnte.

Viele Untersucher haben versucht die phototropische Intensitätsschwelle zu bestimmen. Aus den Beobachtungen Wiesners, Figdors, Oltmanns', v. Guttenbergs und Richters ${ }^{1}$ ) geht hervor bei welchen äuszerst schwachen Intensitäten noch eine phototropische Krümmung $z u$ erhalten ist. Experimentell ist es auch hier nicht gelungen die Schwelle zu bestimmen, da das Zustandekommen eines Effektes sehr abhängig von allen Umständen sein wird, welche Einflusz haben auf die Stärke der Perzeption wie die Temperatur und die Reinheit der Luft. Es musz auszerdem noch von Einflusz sein ob die Pflanzen auf einem Klinostaten der Gegenwirkung der Schwerkraft entzogen sind oder dasz der Krümmung vom Anfange an entgegengearbeitet wird, wie lange die Beleuchtung fortgesetzt wird und wie schwach die Krümmungen sind welche wahrgenommen werden.

Das schwierigste Problem ist die Frage ob man eine Energieschwelle annehmen soll. Vorausgesetzt musz werden, dasz für das Auge ein Schwellenwert für die kleinste wahrnehmbare Krümmung besteht. Es ist aber die Frage ob auch für die Pflanze eine dergleiche Schwelle besteht. Im experimentellen Teile dieser Untersuchung (ई 12) ist schon darauf hingewiesen worden, dasz es nicht möglich ist die Energiemenge festzustellen, wo zuerst eine Krüm-

1) Richter, O. Beispiele ausserordentlicher Empfindlichkeit der Pflanzen. Wien. 1912.

Richter, O. Ueber die Steigerung der heliotropischen Empfindlichkeit von Keimlingen durch Narkotika. Sitz. ber. Ak Wien. Bd. 121, 1912. 
mung auftritt, da nie die Sicherheit besteht, dasz sich ein Reiz nicht in einer Reaktion äuszert. Die Frage, welche der kleinste Reiz ist der noch eine Reaktion veranlaszt, kann nicht mit Frucht studiert werden da diese Schwellenwerte jedenfalls experimentell nicht bestimmt werden können. Darum ist es ein rein theoretisches Problem ob dergleichen Schwellen bestehen. Da aber über die Prozesse, welche nach der Perzeption stattfinden, noch gar nichts bekannt ist und es nicht ausgeschlossen werden kann, dasz schon das erste Glied der Kette derselbe Prozesz ist der sich auch in einer Krümmung äuszert, ist es vorsichtiger über die theoretische Möglichkeit des Bestehens von Schwellenwerten auf diesem Augenblicke kein Urteil auszusprechen ${ }^{3}$ ).

\section{$\S 43$. Die Präsentationszeit.}

Der von $\mathrm{Czapek}$ in die botanische Reizphysiologie eingeführte Begriff Präsentationszeit hat schon zu vielen Schwierigkeiten Veranlassung gegeben. Anfangs von ihm definiert als „die kleinste Reizungsdauer, welche noch eine Reflexsbewegung erzeugt" ist die Definition von Pfeffer genauer gestellt als „die Zeitdauer, welche nötig ist um eine Erregung $\mathrm{zu}$ induciren, die nach der Sistirung des Reizes eine eben merkliche Nachwirkungsbewegung zur Folge hat."

Fitting hat darauf hingewiesen, dasz die Präsentationszeit nicht die Reizzeit ist, welche bis zu der Auslösung der Prozesse, welche zur Reaktion führen, verlaufen musz. Diese fangen nach ihm schon beim Beginn der Reizung an. Seine Definition, welche das Wesen des Präsenta-

1) Unter den Physikern besteht eine Strömung, welche eine Diskontinuität der Energie annimmt und auf Grund dessen zu dem Bestehen von Schwellenwerten schlieszen musz. Es besteht aber geringe Aussicht dasz diese Werte jemals für Reaktionen bei Pflanzen bestimmt werden können. 
tionszeit geben sollte ist "die Zeit, während deren ein Reizanlasz wirksam sein musz damit die ausgelösten reaktiven Vorgänge nicht innerhalb der Reaktionszeit für die Krümmung so weit ausklingen, dasz eine sichtbare Krümmung unterbleibt." Durch die Untersuchungen Blaauws und Fröschels bekam die Präsentationszeit eine ganz andere Bedeutung. Nach ihnen ist sie der Zeitfaktor der Energiemenge, die eine eben sichtbare Krümmung veranlaszt.

Unter diesem Einflusse hat Fit ing ${ }^{1}$ ) seine Definition verbessert und sagt nun von der Präsentationszeit, dasz „sie die Reizstärke bestimmt, die erforderlich ist um die Intensität der Perzeptionsvorgänge auf solche Höhe zu bringen, dasz sie die unsichtbaren Vorläufer der Krümmung bis zur Weckung der sichtbaren Krümmung erstärken lassen."

Nach Fitting gehört also die Präsentationszeit zu der Schwelle. Im vorigen Paragrafen hat es sich gezeigt, dasz die Energieschwelle ein theoretischer Begriff ist, der experimentell nicht $z \mathbf{u}$ bestimmen ist. Hierum is aber auch die Bestimmung der Präsentationszeit experimentell unmöglich. Was man immer getan hat, ist von einem Reiz von bestimmter Grösze bestimmen wie lange er dauern musz um eine Reaktion zu bekommen, die so grosz ist, dasz man sie makroskopisch eben wahrnehmen kann.

Es versteht sich, dasz auf diese Weise nicht die Präsentationszeit bestimmt wird. Das geht auch hieraus hervor dasz verschiedene Untersucher für die Präsentationszeit bei Schwerkraftreizung ganz verschiedene Werte gefunden haben. Das wird natürlich dadurch verursacht dasz der eine noch eine schwächere Krümmung makroskopisch wahrnehmen kann als der andere. Es wü rde a m besten sein, dasz man das Wort Präsentationszeit nicht weiter benutzte. Man wäre dann gezwun-

1) Fitting, H. Tropismen, Handwörterbuch der Naturwissenschaften. 
gen, wie es auch hier stattgefunden hat, anzugeben mit welcher Krümmungsstärke man arbeitet. Auf diese Weise wird eine viel gröszere Uebereinstimmung zwischen den verschiedenen Untersuchungen erreicht werden können.

\section{$\S 44$. Die Reaktionszeit.}

Im Abschnitt I ist darauf hingewiesen worden, dasz der Krümmungsprozesz mit Hilfe eines Mikroskopes früher beobachtet werden kann als bei makroskopischer Wahrnehmung. Auch Pringsheim (2 Mitt. p. 449) hat gefunden, dasz eine phototropische Krümmung bei gewöhnlicher Beobachtungsweise nach etwa 90 Minuten, bei der mit Mikroskop schon nach etwa 25-40 Minuten sichtbar wird. Die hier mitgeteilten Werte sind alle relativ kleiner als die seinigen. Die Verkürzung war von 30 auf 15 bis 20 Minuten. In Uebereinstimmung damit ist auch von andere Untersuchern u. a. $\mathrm{Bach}{ }^{1}$ ), Polowzow ${ }^{2}$ ), Maillefer gefunden worden, dasz auch bei geotropischen Krümmungen eine Abkürzung der sogenannten Reaktionszeit erreicht werden kann, wenn mit einem Mikroskop beobachtet wird. Es ist nicht wunderbar, dasz wenn man die gebräuchliche Definition der Reaktionszeit nimmt als die Zeit, die verläuft vom Beginne der Perzeption bis zum Anfang der Krümmung, man nicht weisz wie man sie bestimmen soll und welchen Wert man auf sie zu legen hat.

Der Zweck der im Abschnitt I mitgeteilten Untersuchungen war den Begriff Reaktionszeit näher zu untersuchen. Nachdem es sich gezeigt hatte, dasz die Zeit,

1) Bach. H. Ueber die Abhängigkeit der geotropischen Präsentations- und Reaktionszeit von verschiedenen Auszenbedingungen. Jahrb. f. wiss. Bot. Bd. 44, 1907.

?) Polowzow, W. Untersuchungen über Reizerscheinungen bei den Pflanzen. 1909. 
welche man sich als Reaktionszeit zu bestimmen gewöhnt hatte, jedenfalls gröszer war als die Reaktionszeit, da die Krümmung schon früher anfängt, ist untersucht worden welchen Wert man dennoch auf die früheren Bestimmungen legen konnte. Dadurch dasz es gelungen ist zu zeigen dasz der ganze Krümmungsverlauf abhängig ist von der Energiemenge womit gereizt worden ist, konnte festgestellt werden, dasz wenn auch die Reaktionszeit, die viele Untersucher bestimmt hatten, nicht die gewünschte Latenzzeit war, es doch vollkommen gestattet war aus diesen Werten Folgerungen zu ziehen über die Stärke der Perzeptionsprozesse. Jede Krümmungszeit welche $z$ u einer Krümmung von bestimmter Stärke gehört kann unter gewissen Bedingungen als Maszstab für die Stärke der Reaktion benutzt werden.

Will man also aus experimentellen Gründen die Krümmungszeit, welche zu einer Krümmung gehört welche eben makroskopisch wahrnehmbar ist als Maszstab benutzen, so kann hiergegen kein Eirwand erhoben werden. Diese Krümmungszeit ist sicher gröszer als die Latenzzeit oder theoretische Reaktionszeit und ist darum hier zur Unterscheidung experimentelle Reaktionszeit genannt worden. Die Bestimmung der theoretischen Reaktionszeit hat sich als unmöglich herausgestellt. Es ist nicht möglich festzustellen ob eine Pflanze noch gar nicht gekrümmt ist. Natürlich kann man sich hierüber theoretische Auffassungen bilden. Tröndle 1) meint, dasz bei einer geotropischen Reizung die Reaktion an der äuszersten Spitze augenblicklich anfange. Auch Maillefer und Polowzow nehmen

1) Tröndle, A. Der zeitliche Verlauf der geotropischen Reaktion und die Verteilung der geotropischen Sensibilität in der Koleoptile. Jahrb. f. wiss. Bot. Bd. 52. 1913 
einen direkten Anfang der Krümmung an. Es wird ganz von den Vorstellungen abhängig sein, welche man sich über die Perzeptionsprozesse geformt hat, ob man eine kürzere oder längere Latenzzeit annimmt oder sie ganz verneinen will. In Ermangelung experimenteller Tatsachen wird hier kein Urteil darüber gegeben werden. Sicher ist es aber nicht empfehlenswert erst anzunehmen dasz duktorische Prozesse stattfinden und daraus wieder zu beweisen, dasz es eine Latenzzeit geben musz wie Fitting das getan hat. Blaauw hat schon die Frage aufgeworfen ob der Augenblick, worauf die Krümmung makroskopisch sichtbar wird, eine besondere Bedeutung hat. Für diese Auffassung spricht viel. Die Krümmung nimmt von diesem Augenblicke ab schneller in Stärke zu. In $\S 3$ ist darauf hingewiesen worden, dasz es wahrscheinlich dadurch verursacht wird dasz die mehr basal gelegenen Zonen an der Krümmung teilzunehmen anfangen. Bei phototropischen Krümmungen ist es durch Spitzenbeleuchtung möglich einen scharfen Unterschied $z u$ machen zwischen der Krümmung, die im beleuchteten Teile selbst auftritt und der welche durch Reizleitung zu stande kommt. Es ist die Krümmung, die auf diese letzte Weise entstanden ist. die den starken Krümmungseffekt zu stande bringt. Bei der Besprechung der Gültigkeit der Produktregel für die negative Krümmung ist darauf hingewiesen worden, dasz die induzierten Excitationen sich noch während langer Zeit summieren können, doch dasz das nicht mehr genau stattfindet bei Reizzeiten welche länger als etwa $25 \mathrm{Mi}$ nuten sind. Vielleicht kann Zusammenhang zwischen dem nicht mehr Summieren und der nach derselben Zeit stärker auftretenden Krümmung bestehen (vergl. § 37 und § 38). Tröndle l) hat die Hypothese verteidigt, dasz es eine

1) Tröndle, A. Ueber die geotropische Reactionszeit. Ber. D. Bot. Ges. Bd. 31. 1913. 
konstante Latenzzeit gebe. Diese Latenzzeit würde der Zeitverlauf sein vom Augenblicke worauf die Schwelle erreicht ist bis zum Augenblicke worauf die Reaktion. anfängt. Er stimmt hierin nicht überein mit $F$ itting. A. Paal und vielen anderen die auf Grund ihrer Beobachtungen ein Ineinanderschieben der perzeptorischen und duktorischen Prozesse annehmen. Um die Bedeutung dieser Vorstellung Tröndles untersuchen zu können ist es nötig erst die Data über die Reaktionszeit näher zu betrachten.

Bei den im Abschnitt I $\$ 11$ mitgeteilten Beobachtungen ist eine merkwürdige Abhängigkeit der experimentellen Reaktionszeit von der Stärke des Reizes ans Licht gekommen. Es erwies sich, dasz die Reaktionszeit bei dem Phototropismus für schwache Reize viel länger ist als für starke. Man hat bei diesen Ergebnissen daran zu denken, dasz die Lichtmengen in kurzer Zeit zugeführt waren, sodasz diese Verlängerung der Reaktionszeit nur von der Grösze des Reizes abhängig ist. Verlängerungen der Reaktionszeit sind in der Literatur wiederholt konstatiert worden, sie können aber durch verschiedene Ursachen hervorgerufen werden, worauf man nicht immer geachtet hat.

Man musz drei verschiedene Weisen von Reizung unterscheiden.

$1^{0}$. die Energiemenge wird in kurzer Zeit zugeführt und man bestimmt, wie die Reaktionszeit von der Energiemenge abhängig ist. In diesem Falle ist Energie (Produkt von Intensität und Reizdauer) $\mathrm{nicht}$ k onst a n t, die Reizdauer ist sehr kurz und konstant. Dieser Fall ist in $\S 11$ für den Phototropismus untersucht und $m a n$ hat die starke Abhängigkeit der Reaktionszeit von der Energiemenge gefunden.

$2^{0}$. man untersucht die Abhängigkeit der Reaktionszeit von der Reizdauer wenn mit konstanter Energiemenge 
gereizt wird. In diesem Falle ist also Energie konstant, aber ihre Zusammensetzung wechselnd (Reizdauer nicht konstant). Hierüber bestehen bei dem Phototropismus keine Data. Für Zentrifugalkraft haben $\mathrm{Bach}$ und Frau Rutten-Pekelharing gefunden, dasz je nachdem die Intensität schwächer ist und die Reizdauer zunimmt die Reaktionszeit verlängert wird. Die Intensität ist in diesem Falle wie ein "limiting factor" für das Zustandekommen der Krümmung.

$3^{\circ}$. man bestimmt die Verlängerung der Reaktionszeit, wenn mit verschiedenen Intensitäten fortdauernd gereizt wird. In diesem Falle ist die Energiemenge nicht konstant, ebensowenig die Weise worauf sie zusammengesetzt ist. (Reizdauernichtkonstant). Das ist der Fall, den Tröndle ') besprochen hat. Bei Fitting $\left.{ }^{2}\right)$ findet man ein Kritik über die Tröndlesche Untersuchung. In einer neuen Mitteilung versucht Tröndle ${ }^{3}$ ) seine frühere Auffassung für Reizung mit Zentrifugalkraft experimentell zu beweisen.

Er hat die Formel aufgestellt $i(t-k)=$ konstant; hierin bedeutet $i$ die Intensität des Reizes, $t$ die Zeit der Reizung bis zu dem Auftreten der Reaktion und $k$ eine Konstante, die Latenzzeit.

Seine Formel beruht auf der Voraussetzung, dasz die Reaktionszeit bei fortdauernder Reizung ebenso lang ist wie bei Reizung während des Schwellenwertes. Das ist sicher nicht richtig für den Phototropismus. Die Reaktionszeit für den Schwellenwert d. h. für die kleinste wahrgenommene Krümmung beträgt hier etwa 100 bis 120 Minuten. Bei fortdauernder Reizung kann diese zu 25 bis 30 Minuten abgekürzt werden. Ein konstanter Faktor

1) Tröndle, A. Der Einfluss des Lichtes auf die Permeabilität der Plasmahaut. Jahrb. f. wiss. Bot. Bd. 48. 1910.

$\left.{ }^{2}\right)$ Fitting, H. Tropismen. Handwörterbuch der Naturw.

3) Tröndle, A. Ber. D. Bot. Ges. Bd. 31. 1913. 
$k$, eine konstante Latenzzeit besteht hier also nicht. Tröndles Formel kann hier also nicht richtig sein.

Es können hier einzelne Zahlen mitgeteilt werden, die beim Hafer bei Schwerkraftreizung erhalten sind. Bei diesen Versuchen, welche mit einem anderen $Z$ wecke stattgefunden haben, ist nicht die Reaktionszeit bestimmt worden, sondern die Stärke der Krümmung 30 Minuten nach dem Anfange der Reizung. Tröndle fand für die Reaktionszeit 34,187 Minuten.

Die Stärke der Krümmung ist bestimmt worden mit einer Millimeterteilung indem die horizontale Abweichung auf $1 / 4 \mathrm{mM}$. geschätzt wurde. Temp. $23^{\circ} \mathrm{C}$.

\begin{tabular}{|c|c|c|}
\hline Reizdauer. & $\begin{array}{l}\text { Krümmungsstärke. } \\
\text { nach } 30 \mathrm{Min} .\end{array}$ & Anzahl Pflanzen. \\
\hline 4 Min. & $0,32 \mathrm{mM}$ & 161 \\
\hline $4 \frac{1}{2}$ Min. & $0,37 \quad$, & 109 \\
\hline $5 \mathrm{Min}$. & 0,39 & 165 \\
\hline 6 Min. & $0,46 \quad$. & 62 \\
\hline
\end{tabular}

Nach 30 Minuten war also die mittlere Stärke der erreichten Krümmung abhängig von der Stärke des Reizes. Daraus folgt, dasz die durch einen stärkeren Reiz verursachte Krümmung schon nach 30 Minuten stärker ist.

Das heiszt, dasz für die schwächste wahrnehmbare Krümmungsstärke noch ein Unterschied in der Reaktionszeit bestehen musz.

Diese Beobachtungen sind nicht im Widerstreit mit der Vorstellung Tröndles dasz eine konstante Latenzzeit verlaufen müsse. Sie zeigen nur dasz so lange man mit Krümmungen von gewisser Stärke arbeitet eine $\mathrm{Ab}$ hängigkeit der Krümmungszeit von der Stärke des Reizes gefunden werden musz. Das erklärt die Beobachtung Rutgers', dasz die Reaktionszeit bei Reizung während 
der Präsentationszeit länger ist als bei Reizung von längerer Dauer. (1. c. p. 97). Als Beschwerde könnte man gegen Tröndles Untersuchung anführen, dasz obwohl er mit einer Krümmung gewisser Stärke gearbeitet hat er nicht angibt wie stark sie war. Auch bei der Bestimmung von Reaktionszeiten musz das immer mitgeteilt werden, da es unmöglich ist mit der reinen Latenzzeit zu arbeiten. Da seine Resultate mit der von vielen Untersuchern verteidigten Auffassung im Widerstreit ist, dasz die Prozesse, welche zu einer Krümmung führen schon durch eine schwächere Reizung als die der Energieschwelle ausgelöst würden, ist es noch nicht möglich sich ein Urteil zu formen über die Bedeutung seiner Formel bei Reizung mit Zentrifugalkraft. Für den Phototropismus hat die Tröndlesche Formel keinen Wert. Viele Untersucher haben darauf hingewiesen, dasz die Reaktionszeit ein sehr schlechter Maszstab ist, wenn man untersuchen will, wie der normal verlaufende Reizvorgang durch verschiedene Umstände beeinfluszt wird. Diese Beschwerden beruhen teils darauf, dasz die Reaktionszeit so variabel und schwierig genau zu bestimmen ist, aber auch darauf, dasz sie in so geringem Masze abhängig ist von der Stärke des Reizes. Wenn man Aenderungen der Reaktionszeit studieren will, musz man insbesondere Rechnung tragen mit den drei obengenannten Fällen wodurch eine Verlängerung der Reaktionszeit veranlaszt werden kann. Bei vergleichenden Versuchen wird man immer dafür sorgen müssen, dasz die Verlängerung nicht durch die längere Reizdauer verursacht wird. Damit hat man nicht immer genügend Rechnung getragen. So ist z. B. die Verlängerung der Reaktionszeit die Rutgers bei sehr tiefen Temperaturen fand ohne $Z$ weifel für einen $T$ eil durch die Verlängerung der Reizdauer verursacht. (vergl. auch Bach). Nur wenn der Reiz in kurzer Zeit zugeführt wird kann man aus einer verlängerten Reaktionszeit etwas über 
Aenderungen die in der Pflanze unter dem Einflusse sehr hoher Temperatur oder verdünnter Luft stattgefunden haben ableiten. (vergl. A. Paali)).

Bei dem Phototropismus würde für das Bestimmen des Einflusses äuszerer Umstände auf dem Perzeptionsprozesse die experimentelle Reaktionszeit benutzt werden können, wenn man nur in dem Gebiete bleibt wo sie stark abhängig ist von der Stärke des Reizes. Da aber Bestimmungen der Reaktionszeit experimentell viel schwieriger auszuführen sind als die der nach einer bestimmten Zeit erreichten Krümmungsstärke so sind mit dieser Methode keine Vorteile verbunden.

Wenn die Umstände unter welchen der Reizvorgang verläuft ungeändert bleiben und das Produkt von Intensität und Zeit konstant gehalten wird, also nur die Intensität und die Reizdauer in Stärke wechseln, wird, wie oben sub 2 beschrieben worden ist, durch die schwächere Intensität eine Verlängerung der Reaktionszeit auftreten. Das haben Frau Rutten-Pekelharing und $\mathrm{Bach}$ auch wirklich bei Reizung mit Zentrifugalkraft gefunden. Sehr merkwürdig aber ist es dasz erstgenannte bei der Untersuchung des Sinusgesetzes für den Geotropismus eine Verkürzung der Reaktionszeit in einigen Lagen gefunden hat wo die Intensität des Reizes schwächer ist. Wenn das bestätigt wird so würde man hier in der Reaktionszeit ein Mittel besitzen um auszumachen ob bei Reizung in verschiedenen Lagen der Reizvorgang qualitativ anders verläuft. (Vergl. auch § 38).

Viel verwickelter wird das Problem der Reaktionszeiten wenn verschiedene Krümmungen zusammenwirken. Bei der Untersuchung über den Einflusz mehrseitiger Beleuchtungen ist die Zeit nach welcher die Krümmungen sichtbar

1) Paal, A. Analyse des geotropischen Reizvorgangs mittels Luftverdünnung. Jahrb. f. wiss. Bot. Bd. 49. 1911. 
werden auszer Betracht geblieben. Man kan hier nur schwer von Reaktionszeiten sprechen da der Augenblick worauf eine Krümmung sichtbar wird durch den ganzen Komplex der gleich oder entgegengesetzt gerichteten Reize bestimmt wird. Die vielen von $\mathrm{Pr}$ ing sheim eingeführ ten Namen sind hier nicht weiter analysiert worden, da es sich gezeigt hat dasz der Standpunkt von woher er sie eingeführt hat weniger richtig ist. Die Tatsachen, die er über Verkürzung und Verlängerung der Reaktionszeit konstatiert hat sind mit Hilfe der in Teil I und II erhaltenen Data ganz gut zu verstehen.

$\S 45$. Verteilung der Empfindlichkeit über die Pflanze.

Die im Abschnitt IV erreichten Resultate geben Veranlassung auf die Unterschiede in Reaktionsvermögen zwischen Basis und Spitze hinzuweisen. Bei der Basis wird ein schwacher Effekt viel schwieriger beobachtet als bei der Spitze da das Auge viel empfindlicher ist für die Unterscheidung der Richtungsänderung von Vorder - und Hinter seite der Spitze in Bezug auf einander als der mehr absoluten Lageänderung bei schwachen basalen Krümmungen. Im letzten Falle wird die Pflanze eine kleine Abweichung von der vertikalen Lage zeigen. Diese Lageänderung ist schwer zu beobachten, da sie nicht mit einer anderen genau vertikal bleibenden Linie verglichen werden kann. $\mathrm{Ob}$ für Spitze und Basis die Cardinalpunkte (der Anfang der positiven Reaktion die maximale positive Krümmung und der Anfang der negativen Krümmung) bei denselben Energiemengen gelegen sind, ist auf Grund der erhaltenen Resultate noch nicht $z u$ entscheiden.

Man wird mit der Möglichkeit Rechnung tragen müssen, dasz die so viel schwächeren Reaktionen der Basis nicht verursacht werden durch eine geringere quantitative Emp- 
findlichkeit des Perzeptionsapparates sondern dasz entweder das Reaktionsvermögen relativ geringer ist oder dasz wie Fitting ${ }^{1}$ ) sich vorstellt ,der polare für die Induction des Phototropismus charakteristische Gegensatz" schwieriger in den basalen Zonen zustande kommt. Die erste Möglichkeit ist weniger wahrscheinlich da die selben basalen Zonen bei Reizen in langer Zeit zugeführt und ebenso bei Krümmungen welche aus der Spitzenzone nach der Basis fortgeleitet werden das Vermögen haben sich sehr deutlich und stark zu krümmen.

Ueber die Empfindlichkeitsverteilung für Geotropismus und Zentrifugalkraft sind bei Avena von $\mathrm{Tröndle} \mathrm{und}$ v. Guttenberg ${ }^{2}$ ) Untersuchungen verrichtet worden. Von Guttenberg versuchte sie mit der Methode Piccard-Haberlandt zu bestimmen wobei Spitze und Basis zu gleicher Zeit entgegengesetzt gereizt werden. Er kam zu dem Resultate, dasz die Spitzenzone am empfindlichsten ist, dasz aber auch die basalen Zonen, wenn auch in geringerem Masze, auf einen Reiz reagieren. Ob man aus diesen Versuchen etwas über eine Empfindlichkeitsverteilung in der Pflanze ableiten darf ist davon abhängig was man unter Empfindlichkeit verstehen will. Wenn man als ihr Masz die Lage der obengenannten Cardinalpunkte annimmt, so ist esnicht gestattet aus Guttenbergs Versuchen zu einer geringeren Empfindlichkeit zu schlieszen da er nur Unterschiede in Reaktionsstärke verglichen hat. Nimmt man aber die Stärke der Krümmung, die durch eine bestimmte Energiemenge verursacht wird als Maszstab, dann kann man sich ganz mit seiner Auffassung vereinigen.

Tröndle hat aus seinen Beobachtungen über yeotro-

1) Fitting, H. Lichtperzeption und phototropische Empfindlichkeit. Jahrb. f. wiss. Bot. Bd. 45. 1908.

2) Guttenberg, H. Ritter von. Ueber die Verteilung der geotropischen Empfindlichkeit in der Koleoptile der Gramineen. Jahrb. f. wiss. Bot. Bd. 50. 1911. 
pische Krümmungen bei Avena abgeleitet, dasz die Empfindlichkeit einer Zone umgekehrt proportional ist mit der Entfernung von der Spitze. Er hat aber nicht berücksichtigt, dasz auch bei dem Geotropismus eine Krümmung der Basis teilweise verursacht werden kann durch einen Reiz der in der Spitze perzipiert worden ist und durch Reizleitung in die Basis angelangt ist. Darum ist es erwünscht bei seinen Folgerungen Vorsicht zu üben.

§46. Ueberden Einfluszder Lichtrichtung.

Da $\mathrm{Mast}^{1}$ ) und Noack vor kurzer Zeit Betrachtungen über die zahlreichen Beobachtungen geliefert haben die über dieses Problem verrichtet sind, kann hier eine kurze Bemerkung genügen. Einander stehen zwei Auffassungen gegenüber, die Sachs', die annimmt dasz die Richtung des Lichtes in der Pflanze perzipiert werde und die Oltmanns' u. a. dasz die Pflanze den Lichtunterschied an verschiedenen Seiten empfinde. Mast hat die Versuche Oltmanns' wiederholt und durch eine genaue Aufstellung gezeigt, dasz eine Pflanze, die von zwei entgegengesetzten Seiten durch parallele Lichtbündel gereizt wird, wobei auf dem Durchschnitt dieser Bündel die Intensität der Strahlen von der einen Seite nach der anderen abnimmt, sich senkrecht auf der Richtung der Strahlen nach dem Teile der Lichtbündel krümmt wo die Beleuchtungsstärke am gröszten ist. Es ist aber nicht richtig mit $\mathrm{Mast}$ hieraus die Folgerung zu ziehen, dasz die Richtung der Strahlen in der Pflanze von keinem Einflusse sei. Wie aus den in dieser Untersuchung mitgeteilten Versuchen mit mehrseitigen Beleuchtungen hervorgegangen ist (vergl. auch $\left.\mathrm{Hagem}^{2}\right)$ ) wird die

1) Mast, S. O. Light and the Behavior of organisms. 1911.

2) Hagem, O. Ueber die resultierende phototropische Lage bei zweiseitiger Beleuchtung. Berg. Mus. Aarbok. 1911. Heft 1. 
Richtung der Krümmung durch die Resultante der Krümmurgstendenzen der verschiedenen Seiten der Pflanze bestimmt. In jedem Teile, man darf wahrscheinlich wohl sagen in jeder Zelle, musz die Richtung der Krümmung von der Lichtrichtung in diesem Teil oder in dieser Zelle abhängig sein. Ob diese Richtung dadurch perzipiert wird dasz die Stärke der Prozesse an Vorder- und Hinterseite verschieden sind ist noch nicht zu entscheiden. (vergl. B la a u w $\left.{ }^{1}\right)$ ).

Noack hat einen qualitativen Unterschied der Richtung der Lichtstrahlen in der Pflanze angenommen. Im Abschnitte $\mathrm{V}$ ist auseinandergesetzt worden, dasz seine Resultate auch auf eine andere Weise erklärt werden können, wenn man in Anmerkung nimmt, dasz Strahlen, die von oben kommen die im konisch zulaufenden Teil der Spitze gelegenen Zellen mehr senkrecht treffen. Diese sehr empfindlichen Zellen werden dadurch stärker gereizt und verursachen eine stärkere Reaktion als wenn die Pflanze durch horizontale Strahlen getroffen wird. Wenn diese Lösung sich als die richtige erweisen sollte, so würde man künftig auch mit der Lage der Zellen und ihrer Orientation $z u$ einander Rechnung tragen müssen.

\section{$\S 47$. Schluszbetrachtung.}

Im Vorhergehenden ist versucht worden die phototropischen Erscheinungen näher kennen zu lernen, teils indem einige Probleme experimentell untersucht worden sind teils durch das Studium des in der Literatur anwesenden Materiales. Es zeigte sich dasz das Verständnis dieser

1) Blaauw, A. H. De primaire photogroeireactie en de oorzaak van positieve krommingen van Phycomyces nitens. Verslagen Kon. Ak. v. Wetensch. Amsterdam. Dec. 1913. 
Erscheinungen noch äuszerst gering ist. Zwar bestehen eine grosze Anzahl Hypothesen, doch wenn man sich nur auf Grund der Tatsachen eine Vorstellung dieser Prozesse bilden will, so stellt es sich noch als ganz unmöglich heraus.

Eine der gröszten Beschwerden womit man immer wieder $z \mathbf{u}$ kämpfen hat, ist dasz eine Anzahl von Begriffen ihren Ursprung von einem ganz anderen Gebiete herleiten und sich eingebürgert haben bevor man untersucht hat ob wirklich eine Vergleichbarkeit besteht zwischen den phototropischen Erscheinungen und den Prozessen auf welche sie Beziehung haben. Reizschwelle, Präsentationszeit, Reaktionszeit, Erregung, Abklingen, Ermüdung und Stimmung sind nur einige wenige Beispiele. In den vorhergehenden Betrachtungen ist versucht worden die experimentelle Basis dieser Begriffe kennen zu lernen. In vielen Fällen erwies es sich als ebenso schwierig zu,verneinen als $z u$ bestätigen, dasz die Prozesse in der Pflanze stattfinden wie die Untersucher sich es gedacht haben. Es ist noch ganz unmöglich sich eine Idee davon zu bilden welcher Art die Prozesse sind die durch den Lichtreiz in der Pflanze erweckt werden. Selbst über die Natur der Perzeption herrscht noch Unsicherheit, wenn auch die in der Energiehypothese formulierten Ergebnisse in eine bestimmte Richtung deuten. Die Vorstellung Bla a uws, dasz der Perzeptionsprozesz photochemischer Art sein würde, ist sehr anziehend. Warum es dann aber eine photochemische Gleichgewichtsänderung und nicht ein photokatalytischer Prozesz sein würde wie z.B. Lehmann ${ }^{1}$ ) es sich denkt bei der Keimung von Samen unter dem Einflusse des Lichtes, ist von Blaauw nicht mit genügender Sicherheit auseinandergesetzt worden. (vergl. Pringshei $\left.{ }^{2}\right)$ ).

1) Lehmann, E. Ueber die Beeinflüssung der Keimung lichtempfindlicher Samen durch die Temperatur. Zeitschr. für Bot. Bd. 4. 1912.

2) Pringsheim, E. 1. c. 3e mededeeling. 
Die Bedeutung der Produktregel und der Energiehypothese musz aber nicht sosehr gesucht werden in der dadurch gegebenen Möglichkeit zu einer mehr oder weniger oberflächlichen Vergleichbarkeit mit chemischen Prozessen als in der Tatsache dasz hier für das erste Mal eine quantitative Beziehung zwischen dem äuszeren Reize und den in der Pflanze verlaufenden Prozessen gefunden worden ist. In den letzten Jahren sind dergleichen Beziehungen auch für andere Reizreaktionen gefunden worden. Man wird aber für die Zukunft mehr erwarten können von dem Studium der Abweichungen und Ausnahmen. welche von diesem Gesetze bestehen als von einem oberflächlich Feststellen der Gültigkeit eines Reizmengengesetzes bei allen möglichen Prozessen.

Hierfür wird man aber Material nötig haben das zuverlässige Resultate gestattet. Wiederholt hat es sich in dieser Untersuchung gezeigt, dasz die Ergebnisse sehr variabel sein können. Die erhaltenen Resultate haben aber auch den Eindruck gegründet dasz es gelingen wird Ergebnisse zu bekommen welche in viel höherem Masze zuverlässig sind, wenn man mit Pflanzen arbeitet die immer unter denselben Umständen gezogen werden und wenn man die Versuche unter vollkommen vergleichbaren Umständen anstellt.

\section{$\S 48$. Zusammenfassung.}

I. Wenn man eine Avenakeimpllanze mit schwachen Lichtmengen reizt, $(1-100$ M.K.S.) kommt eine positive phototropische Krümmung zustande.

II. Der Augenblick wo die Krümmung anfängt ist experimentell nicht $z u$ bestimmen, daher ist es unmöglich mit der "theoretischen Reaktionszeit" zu arbeiten. $\$ 3 . \S 5$.

III. Die Schwerkraft arbeitet der Krümmung vom Anfang an entgegen. §3. Dadurch entsteht ein Maximum 
(maximale Krümmungsstärke). Eine rein phototropische Krümmung wird nur bei Pflanzen erhalten bei welchen die Wirkung der einseitigen Schwerkraft durch Drehung um die horizontale Achse eines Klinostaten aufgehoben wird. $\S 4$.

IV. Je nachdem die Reizmenge gröszer ist, nimmt auch die Reaktionsstärke zu. $\S 6$.

V. Eine stärkere Reaktion äuszert sich:

a. in einem früher Sichtbarwerden der Krümmung d.h. in einer Abkürzung der experimentellen Reaktionszeit $\$ 11$.

$b$. in dem Erreichen einer stärkeren maximalen Krümmung $§ 6$.

c. in dem Erreichen einer stärkeren Krümmung in einer bestimmten Zeit. $\S 8$.

VI. Eine untere Grenze für das Auftreten von Krümmungen (Schwelle) ist experimentell nicht nachzuweisen. $\S 12$. Die Frage ob eine Schwelle besteht ist rein theoretisch und in diesem Augenblicke nicht zu lösen. $\S 42$.

VII. Auch bei rein phototropischen Krümmungen (III) sind die in $\mathrm{Va}$ und $\mathrm{c}$ besprochenen Beziehungen gültig. $\S 10$.

VIII. Bei Energiemengen welche gröszer sind als 250 M.K.S. nimmt die positive Krümmung an Stärke ab. Die Abnahme kommt insbesondere durch das Unterdrücktwerden der späteren Krümmungsstadia zustande. $\S 13$.

IX. Bei Energiemengen, welche nur wenig gröszer als 2000 M.K.S. sind tritt nach der positiven Krümmung eine negative auf. Je nachdem die Energiemenge stärker wird nimmt die positive Krümmung $a b$ und die negative $z u$, bis nur die negative Krümmung sichtbar wird. $\S 13$.

X. Bei schwachen Energiemengen wobei nicht innerhalb etwa 20 bis 25 Minuten (Reaktionszeit?) die 2000 M.K.S. zugeführt worden sind, tritt keine negative Krümmung mehr auf. $\S 16$.

XI. Eine rein negative Krümmung tritt nur bei Intensitäten stärker als etwa 12 M.K. auf. $§ 14$. 
XII. Bei jeder Lichtintensität, wobei negative Krümmung auftritt kommt bei längerer Dauer der Reizung eine Abnahme der negativen Reaktion zustande, und nimmt die positive wieder an Stärke $z u$, bis sie nur allein auftritt. $\S 17$.

XIII. Bei diesen Lichtintensitäten kann man also eine erste positive und eine zweite positive Reaktion unterscheiden. Ein qualitativer Unterschied liegt aber nicht vor. $\S 17$.

XIV. Es ist noch nicht festgestellt ob das Auftreten der zweiten positiven Krümmung von der Lichtmenge abhängig ist. Jedenfalls wäre das nur möglich bei Intensitäten wobei die für die zweite positive Krümmung benötigte Energiemenge innerhalb 25 Minuten zugeführt wird.

$\mathrm{XV}$. Bei starken Intensitäten ist noch eine zweite Abnahme der positiven Reaktion beobachtet, also eine zweite negative Reaktion. $\S 18$.

XVI. Die Empfindlichkeit der Basis ist viel gröszer als bekannt war. Bei einer Verdunkelung der Spitze von $5 \mathrm{mM}$. tritt bei etwa 100 M.K.S. eine äuszerst schwache positive Reaktion auf. Von 300 bis 1200 M.K.S. sind die Krümmungen ziemlich deutlich. Bei gröszeren in kurzer Zeit zugeführten Energiemengen tritt keine deutliche positive Krümmung mehr auf. Es konnte aber nicht mit Sicherheit festgestellt werden ob negative Krümmungen vorkommen. $\S 21$.

XVII. Starke positive basalinduzierte Krümmungen treten nur bei Beleuchtungen von 15 Minuten und länger auf. $\S 21$.

XVIII. Der Unterschied in der Reaktion von Basis und Spitze besteht in der Schwäche der ersten positiven Reaktion und der Schwierigkeit negative Krümmungen zuverlässig zu zeigen. $\S 21$.

Ob man der Basis eine geringere Empfindlichkeit zuschreiben will hängt davon $a b$ was man unter Empfindlichkeit versteht, die Lage der Cardinalpunkte oder die Stärke 
der Krümmung auf einen Reiz von bestimmter Grösze. $§ 45$.

XIX. Ein Einflusz von Beleuchtungen der Basis auf Spitzenkrümmungen konnte nur in so weit beobachtet werden als die in der Spitze induzierte Krümmung in den basal vorbeleuchteten Zonen einen geringeren Effekt zufolge hat. $\mathrm{Ob}$ die Krümmung in der nicht vorbeleuchteten Spitzenzone selber durch eine akropetale Reizleitung geringer ist konnte nicht festgestellt werden. $\S 22$.

$X X$. In Uebereinstimmung mit der Untersuchung No acks wurde gefunden dasz der Einflusz der Strahlenrichtung nicht nach dem Sinusgesetze berechnet werden kann. §23.

XXI. Die Erklärung wäre zu finden in der Form der Spitze wenn man in Anmerkung nimmt dasz Strahlen die quer von oben kommen die im konischen Teile der Spitze gelegenen Zellen mehr senkrecht treffen. § 23.

XXII. Die Versuche Masts beweisen nicht dasz die Richtung der Strahlen in der Pflanze ohne Einflusz ist. Die Krümmungsrichtung eines Avenakeimlinges wird durch die Resultante aller vorhandenen Krümmungstendenzen bestimmt. § 46 .

XXIII. Bei gieichzeitiger Beleuchtung von zwei entgegengesetzten Seiten reagiert die Pflanze alsob sie die zwei Reize jeden für sich perzipiere. §25. Die Pflanze perzipiert also nicht die Differenz oder das Verhältnis der Energiemengen. Die zustandekommende Krümmung ist die Resultante der erweckten Krümmungstendenzen.

XXIV. Eine positive Krümmung hinsichtlich der einen Lichtquelle kann sich mit einer negativen hinsichtlich der entgegengesetzten nicht $z u$ einem stärkeren Effekt kombinieren. $\S 25$.

XXV. Finden zwei gleich starke Beleuchtungen von zwei entgegengesetzten Seiten nach einander statt so kann sich jede desto stärker in einer Krümmung äuszern, je nachdem die Zeit zwischen ihrem Anfang gröszer ist. $§ 26$. 
Im allgemeinen tritt bei aufeinanderfolgender Beleuchtung von zwei entgegengesetzten Seiten die durch die zweite Beleuchtung induzierte positive Krümmung stärker auf als die durch die erste Beleuchtung induzierte positive Krümmung. Eine negative Krümmung hinsichtlich der zweiten Beleuchtung wird aber durch eine vorhergehende Beleuchtung der anderen Seite von gewisser Dauer unterdrückt.

Es ist nicht möglich festzustellen ob die nach der Lichtquelle der zweiten Beleuchtung gerichtete Krümmung nur eine positive ist hinsichtlich der zweiten Beleuchtung oder auch noch ein negatives Element hinsichtlich der ersten Beleuchtung enthalten kann. $§ 26$.

XXVI. Eine gleichzeitige Beleuchtung von zwei einander entgegengesetzten Seiten mit folgender oder vorhergehender einseitiger Beleuchtung musz als eine Kombination einseitiger Reize aufgefaszt werden, von welchen eine während längerer Zeit fortgesetzt wird. Der Unterschied in den zwei Beleuchtungsweisen besteht darin dasz im ersten Falle die Beleuchtungen gleichzeitig, im zweiten nach einander anfangen. Das hat zufolge dasz im zweiten Falle die Krümmungen nach der Lichtquelle der kurzdauernden Beleuchtung stärker auftreten. Es ist noch nicht gelungen hinsichtlich dieser letzteren Krümmungen festzustellen wie sie aufgefaszt werden müssen. $\S 27$.

XXVII. Bei allseitigen Vor - und Nachbeleuchtungen erzielt man dieselben Resultate wie bei zweiseitigen; auch hier musz die Lichtmenge der einseitigen Beleuchtung der an dieser Seite schon bei der allseitigen Beleuchtung zugeführten Energie hinzugefügt werden. § 30.

XXVIII. Eine schwache allseitige Vorbeleuchtung hat also zur Folge dasz;

$1^{0}$. um eine positive Krümmung zu bekommen eine gröszere Energiemenge zugeführt werden musz als bei einer nicht vorbeleuchteten Pflanze, da die an der nicht nachbeleuchteten Seite zugeführte Energie der Krümmung entgegenwirkt, 
$2^{0}$. um eine negative Krümmung zu bekommen weniger Energie zugeführt zu werden braucht, da auch die bei der allseitigen Vorbeleuchtung an der später einseitig beleuchtet werdenden Seite zugeführte Energie mitwirkt.

Auch die bei stärkeren und länger dauernden allseitigen Vorbeleuchtungen auftretenden Erscheinungen können nach Analogie der zweiseitigen Beleuchtungen erklärt werden. $\S 30$.

XXIX. Da die Reaktion eines Avenakoleoptyls als die Resultante einer groszen Ahzahl selbständig perzipierender und mehr oder weniger unabhängig von einander reagierender Teile aufgefaszt werden musz, hat man zwei Stimmungserscheinungen $z u$ unterscheiden, erstens die Rezeptions- und Aktionsfähigkeit jeder Teilreaktion, zweitens den Einflusz dieser Teilreaktionen auf einander. $\S 40$.

XXX. Wenn nach der allseitigen Vorbeleuchtung die Pflanzen einige Zeit im Dunklen gelassen werden, bevor man sie einseitig reizt, kann man untersuchen wie die durch die Vorbeleuchtung entstandene Reaktionsfähigkeit abklingt. Der Einflusz einer allseitigen Vorbeleuchtung erlischt ziemlich schnell so dasz eine eine Stunde später anfangende einseitige Reizung fast ungestört verläuft. §32.

XXXI. Wenn einer allseitigen Beleuchtung eine einseitige vorhergeht, so treten genau dieselben Erscheinungen auf als bei einer zweiseitigen Beleuchtung der eine einseitige vorhergeht. $\S 31$.

Folgt die allseitige Beleuchtung nich sogleich der einseitigen so tritt die Erscheinung, dasz erst eine Krümmung im Sinne der einseitigen Beleuchtung und später eine in der entgegengesetzten Richtung auftritt noch schärfer hervor. §34.

XXXII. Die Energiehypothese sagt, dasz die Stärke der primairen Aenderung welche in der Pflanze durch das Licht zustande kommt, bestimmt wird durch die zugeführte Energiemenge. $\S 37$.

XXXIII. Die Produktregel sagt, dasz um einen bestimmten Effekt zu bekommen es gleichgültig ist ob eine Ener- 
giemenge in kurzer oder langer Zeit zugeführt wird. Die Produktregel hat nur eine beschränkte Gültigkeit. § 37.

XXXIV. Ueber das Abklingen des durch das Licht in der Pflanze hervorgebrachten Zustandes kann man sich zwei Vorstellungen machen, erstens dasz ein Teil der primairen Aenderung verschwindet ohne zu dem Effekt mitgewirkt zu haben; zweitens, dasz die primaire Aenderung nur in so weit verschwindet als sie zu dem Effekt mitwirkt. Die erste Auffassung ist, da das Abklingen ziemlich schnell stattfindet schwierig in Uebereinstimmung zu bringen mit der groszen Gültigkeit der Produktregel. Die Data welche in der Literatur zu finden sind über ein schnelles Verschwinden der primairen Aenderung ohne zu dem Effekt mitzuwirken sind nicht beweisend. § 38 .

XXXV. Die Prozesse, welche in dieser Untersuchung als Abklingprozesse besprochen sind, beruhen alle auf der Erscheinung, dasz zwei Reize von entgegengesetzten Seiten einander weniger entgegenwirken je nachdem die Zeit zwischen ihrem Anfange gröszer ist. Wenn also eine allseitig vorbeleuchtete Pflanze sich einem Reiz, der eine Stunde später anfängt, gegenüber wie eine unbeleuchtete Pflanze verhält so.darf man daraus nicht die Folgerung ziehen dasz die Folgen der ersten Reizung ganz verschwunden sind.

XXXVI. Da die Präsentationszeit zu der Schwelle gehört und diese nur theoretischen Wert hat wäre es erwünscht nicht weiter bei einer eben sichtbaren Krümmung von Präsentationszeit zu reden, sondern von der bei einer Krümmung bestimmter Stärke gehörenden Reizdauer. § 43.

XXXVII. Bei dem Phototropismus ist es nicht möglich festzustellen ob eine Reaktionszeit besteht, Es ist aber sehr wahrscheinlich, dasz im dem Augenblicke wo die sichtbare Krümmung auftritt eine neue Phase im Krümmungsprozesse erreicht wird. $\S 44$. 
XXXVIII. Die von Tröndle abgeleitete Formel $i(t-k)=$ konstant ist für den Phototropismus nicht gültig. § 44 .

\section{TAFELERKLÄRUNG}

Fig. 1. Reizdauer 14 Min. Beleuchtungsstärke 5.5 M.K. Temp. 23 C. Starke positive Krümmung. Die Pflanzen 1 und 4 von rechts zeigen eine negative Asymmetrie der Spitze. Nach der Beleuchtung auf dem Klinostaten. $2 \frac{1}{2}$ Stunde nach dem Anfange der Beleuchtung photographiert.

Fig. 2. Reizdauer 6 Min. Beleuchtungsstärke 5 M.K. Temp. $23^{\circ}$ C. Starke positive Krümmung. Nach der Beleuchtung auf dem Klinostaten. $2 \frac{1}{2}$ Stunde nach der Beleuchtung photographiert.

Fig. 3. Beleuchtung auf dieselbe Weise als in Fig. 2. $\mathrm{Nach}$ der Beleuchtung nicht auf dem Klinostaten. Die Spitzen sind geotropisch

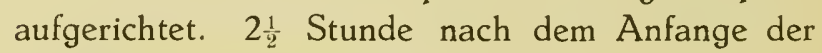
Beleuchtung photographiert.

Fig. 4. Reizdauer 45 Minuten. Beleuchtungsstärke 5.5 M.K. Temp. $23^{\circ}$ C. Kreisförmige positive Kr ümmung. Nach der Beleuchtung auf dem Klinostaten. 4 Stunden nach dem Anfange der Beleuchtung photographiert.

Fig. 5. Nur die Basis von links mit 200 M.K.S. (10 Sek.) gereizt. Die Spitze in einem Käppchen von $5 \mathrm{mM}$. $23^{\circ}$ C. Schwache positive Kr ümmung der Basis. 2! Stunde nach dem Anfange der Beleuchtung photographiert. 
Fig. 1.

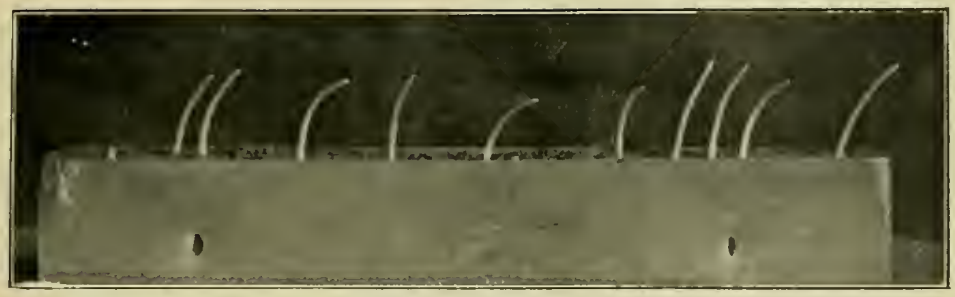

Fig. 2.

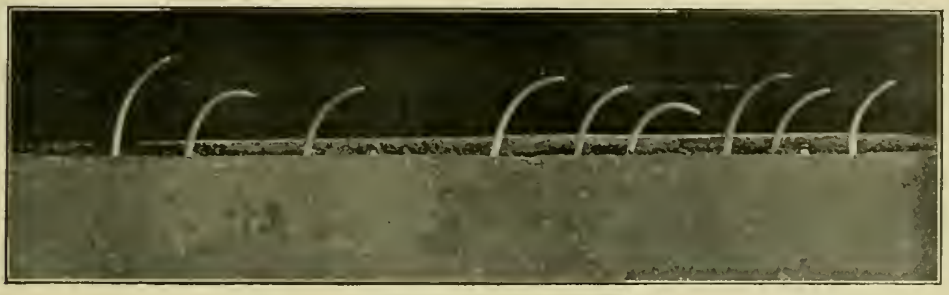

Fig. 3.

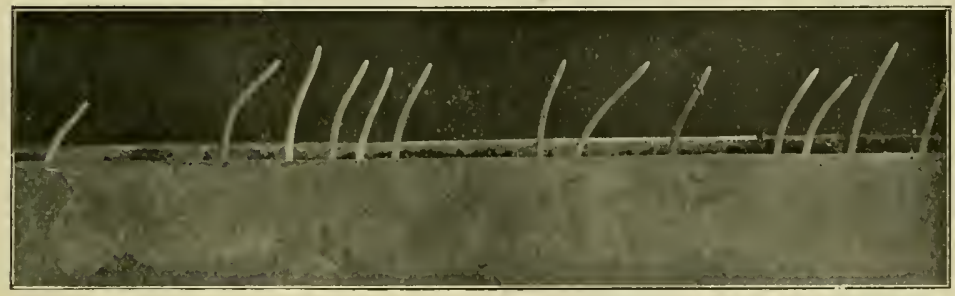

Fig. 4.

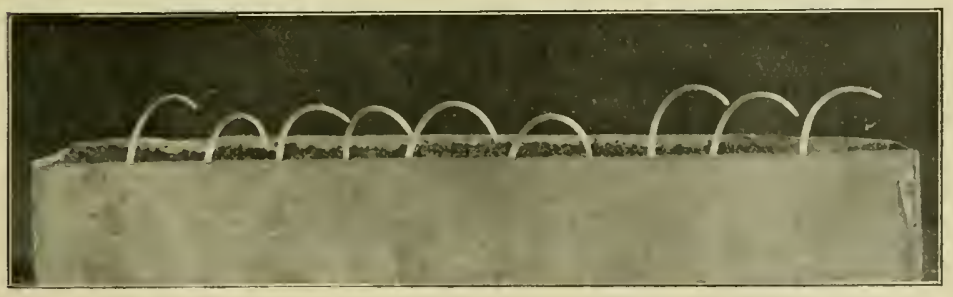

Fig. 5.

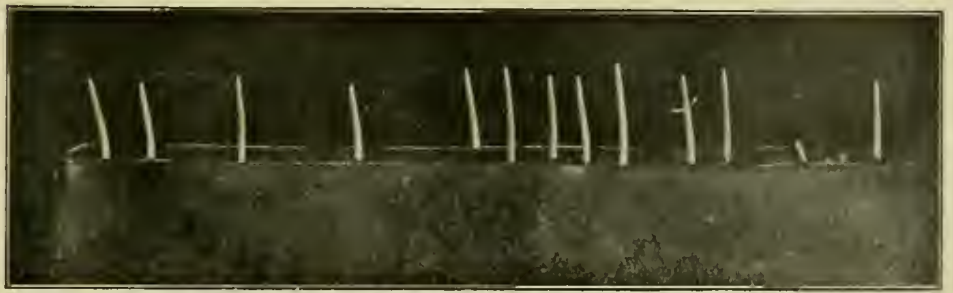



Seite.

Teil I. Einseitige Beleuchtung . . . . . . . . 44

Abschnitt I. Die positive Reaktion . . . . . . . 44

\$ 1. Einleitung . . . . . . . . . . . . 44

$\S 2$. Allgemeine Methode. . . . . . . . . 45

$\S 3$. Der Verlauf einer positiven Krümmung . . 51

$\S 4$. Aufhebung der geotropischen Gegenkrümmung 56

$\S 5$. Reaktions und Krümmungszeit . . . . . 59

$\$ 6$. Abhängigkeit der maximalen Krümmung von der Stärke des Reizes . . . . . . . 60

§ 7. Krümmungszeit der Maximalkrümmung . . 64

§ 8. Die Stärke der Krümmung nach einer bestimmten Zeit . . . . . . . . . . . 65

§ 9. Gleichartige und ungleichartige Krümmungen 65

$\S 10$. Abhängigkeit der Krümmungsstärke von der Energie bei nicht durch Schwerkraftwirkung beeinfluszten Krümmungen . . . . . . 67

$\S 11$. Abhängigkeit der experimentellen Reaktionszeit von der Reizstärke . . . . . . . . 70

$\S 12$. Schwellenbestimmung. . . . . . . . 71

Abschnitt II. Die negative Reaktion . . . . . 73

$\S 13$. Die negative Reaktion . . . . . . . 73

$\$ 14$. Die Energie wobei eine negative Krümmung auftritt. . . . . . . . . . . . . 77

$\S 15$. Die Gültigkeit der Produktregel für die negative Krümmung . . . . . . . . . . . . 84

§ 16. Das nicht Auftreten negativer Krümmungen bei schwachen Intensitäten. . . . . . . 86

$\S 17$. Die zweite positive Krümmung . . . . . 88 
Seite,

$\S 18$. Eine zweite Abnahme der Stärke der positiven Reaktion bei starken Intensitäten

$\S 19$. Einflusz der Temperatur auf die negative Krümmung . . . . . . . . . . . . 92

Abschnitt III. Die Empfindlichkeit der Basis . . . 94

$\S 20$. Methode . . . . . . . . . . . . . 94

$\S 21$. Positive und negative in der Basis induzierte Krümmungen . . . . . . . . . . . 97

$\S 22$. Ueber den Einflusz von Beleuchtungen von Spitze und Basis auf einander . . . . . 102

Abschnitt IV . . . . . . . . . . . . . . 108

$\S 23$. Parallele unter verschiedenen Winkeln einfallende Strahlen . . . . . . . 108

Teil II. Mehrseitige Beleuchtungen . . . . . . 114

$\S 24$. Einleitung . . . . . . . . . . . . 114

Abschnitt V. Zweiseitige Beleuchtungen . . . . 115

$\S 25$. Gleichzeitige Beleuchtung von zwei entgegengesetzten Seiten . . . . . . . . 115

$\S 26$. Zwei Beleuchtungen von entgegengesetzten Seiten nach einander. . . . . . . . 120

$\S 27$. Gleichzeitige Beleuchtung von zwei entgegengesetzten Seiten, der eine einseitige folgt oder vorhergeht . . . . . . . . . . . 129

Abschnitt VI. Allseitige Beleuchtungen . , . . 136

$\S 28$. Einleitung . . . . . . . . . . . . 136

$\S 29$. Methode . . . . . . . . . . . . 137

$\S 30$. Eine allseitige Beleuchtung der eine einseitige folgt. . . . . . . . . . . . . . 141

$\S 31$. Eine allseitige Beleuchtung, der eine einseitige vorhergeht . . . . . . . . . . . 153

Abschnitt VII. Das Abklingen . . . . . . . . 156

$\S$ 32. Das Abklingen einer allseitigen Beleuchtung. 156

$\S 33$. Eine einseitige Beleuchtung, der eine einseitige der entgegengesetzten Seite nicht sogleich folgt 160 
Seite.

$\S 34$. Eine einseitige Beleuchtung der eine allseitige nicht sogleich folgt. . . . . . . 161

§ 35. Zusammenfassung . . . . . . . . . . 163

Teil III. Theoretische Betrachtungen . . . . . . 165

$\S$ 36. Die Kompliziertheit der Reizprozesse . . . 165

$\S 37$. Die Energiehypothese und die Produktregel 167

$\S 38$. Das Abklingen . . . . . . . . . . . 176

§39. Webersches Gesetz . . . . . . . . . 185

$\S 40$. Stimmung. . . . . . . . . . . . 186

$\S 41$. Die negative Krümmung . . . . . . 188

$\S$ 42. Die Reizschwelle . . . . . . . . . 189

$\S$ 43. Die Präsentationszeit. . . . . . . . . 191

§ 44. Die Reaktionszeit . . . . . . . . . 193

$\S 45$. Verteilung der Empfindlichkeit über die Pflanze 201

$\S$ 46. Ueber den Einflusz der Lichtrichtung . . . 203

\$ 47. Schluszbetrachtung . . . . . . . . 204

§ 48. Zusammenfassung . . . . . . . . . . 206 






35185000756021 
